
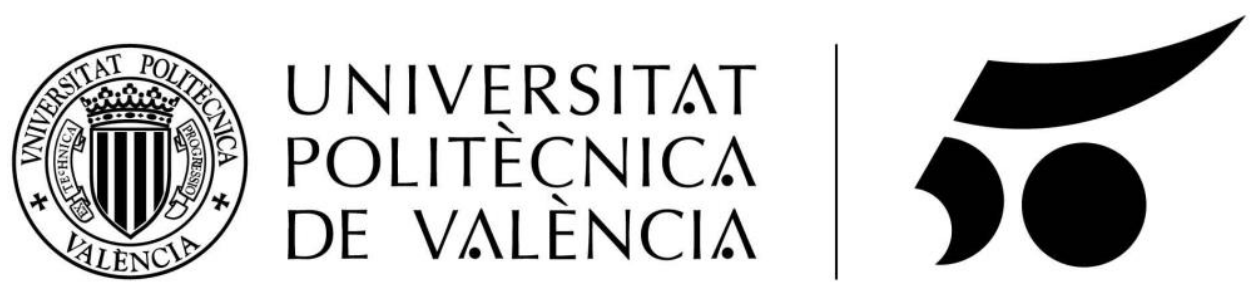

\title{
anys
}

Universitat Politècnica de València

Departament de Matemática Aplicada

A thesis submitted for the degree of Doctor of Philosophy

\section{Application of the self-consistent method of moments to the investigation of dynamic and optical characteristics of plasmas}

\author{
Denis Yu. Dubovtsev
}

Supervisor

Dr. I. M. Tkachenko Gorski

Valencia

July, 2019 
Denis Yu. Dubovtsev. Application of the self-consistent method of moments to the investigation of dynamic and optical characteristics of plasmas (C) July, 2019 


\section{ABSTRACT}

The method of moments occupies a special place among the theoretical methods dedicated to the study of systems with Coulomb interaction between particles. Its essence lies in the fact that the system linear response function is parameterized as a fractionallinear transformation of a (Nevanlinna) parameter function (NPF) with certain mathematical properties. The zero-frequency approximation is applied to determine the latter which permitted to relate it, on the basis of justified physical considerations, to the moments themselves. This NPF static approximation is shown to be consistent within the Shannon entropy maximization method.

In the present work, the self-consistent version of the method of moments is applied to the investigation of the dynamic local field correction and other dynamic characteristics of classical strongly coupled one-component systems, such as dense Coulomb and Yukawa plasmas. The self-consistency of the approach means that the dynamic properties are obtained without any data input from simulations so that the dielectric function satisfies the first five sum rules automatically. Moreover, the dynamic structure factor, dispersion and the dynamic local-field correction are determined using exclusively the static structure factor calculated from the hypernetted chain approximation. A good quantitative agreement with molecular dynamics simulation data is achieved.

In addition, little discrepancy is observed in the plasma dynamic characteristics calculated with the static structure factors, obtained within various methods of calculation of the static structure factor, namely, the hyper-netted chain approximation (HNC), the modified HNC (MHNC) and the variational modified HNC (VMHNC). This stability implies the robustness of the present approach.

Possibilities to abandon the NPF static approximation are analyzed as well. 


\section{RESUMEN}

El método de los momentos ocupa un lugar especial entre los métodos teóricos dedicados al estudio de los sistemas con interacción de Coulomb entre partículas. Lo más importante y característico es el hecho de que la función de respuesta lineal del sistema está parametrizada a semejanza de una transformación lineal fraccionaria de una función de Nevanlinna (NPF, Nevanlinna Parameter Function) bajo ciertas propiedades matemáticas. La aproximación de frecuencia cero se aplica para determinar la última que permitió relacionarla con su momento, teniendo en cuenta aspectos físicos que lo justifiquen. Se muestra que esta aproximación estática NPF es consistente con el método de maximización de entropía de Shannon.

El presente trabajo constituye una versión autoconsistente del método de los momentos para su aplicación a la investigación de la corrección dinámica de campo local, entre otras características dinámicas, de los sistemas clásicos fuertemente acoplados de un componente, como son los plasmas densos de Coulomb y Yukawa. El modelo es autoconsistente ya que las propiedades dinámicas se obtienen sin ninguna introducción de datos obtenidos en las simulaciones, de modo que la función dieléctrica satisface las primeras cinco reglas de suma automáticamente. Además, tanto el factor de estructura dinámico, como la dispersión y la corrección dinámica del campo local, se determinan utilizando exclusivamente el factor de estructura estático calculado a partir de la aproximación de la cadena hiper enlazada. Se muestra que se logra un buen ajuste cuantitativo con los datos de simulaciones de dinámica molecular.

De igual manera, se observa poca discrepancia entre las características dinámicas del plasma calculadas a través de los factores de estructura estática, frente a los obtenidos por otros métodos de cálculo de ese factor de estructura estática, como son la aproximación de cadena hiper enlazada (HNC, Hiper-Netted Chain), la HNC modificada (MHNC, Modified Hiper-Netted Chain) y la HNC modificada variacionalmente (VMHNC, Variational Modified Hiper-Netted Chain). Esta estabilidad implica la robustez del enfoque que se presenta.

Asimismo, se analizan las posibilidades de abandonar la aproximación estática NPF. 


\section{RESUM}

El mètode dels moments ocupa un lloc especial entre els mètodes teòrics dedicats a l'estudi dels sistemes amb interacció de Coulomb entre partícules. El més important i característic és el fet que la funció de resposta lineal del sistema està parametritzada a semblança d'una transformació lineal fraccionària d'una funció de Nevanlinna (NPF, Nevanlinna Parameter Function) sota certes propietats matemàtiques. L'aproximació de freqüència zero s'aplica per a determinar l'última que va permetre relacionar-la amb el seu moment, tenint en compte aspectes físics que ho justifiquen. Es mostra que aquesta aproximació estàtica NPF és consistent amb el mètode de maximització d'entropia de Shannon.

El present treball constitueix una versió autoconsistente del mètode dels moments per a la seua aplicació a la investigació de la correcció dinàmica de camp local, entre altres característiques dinàmiques, dels sistemes clàssics fortament acoblats d'un component, com són els plasmes densos de Coulomb i Yukawa. El model és autoconsistent ja que les propietats dinàmiques s'obtenen sense cap introducció de dades obtingudes en les simulacions, de manera que la funció dielèctrica satisfà les primeres cinc regles de suma automàticament. A més, tant el factor d'estructura dinàmic, com la dispersió i la correcció dinàmica del camp local, es determinen utilitzant exclusivament el factor d'estructura estàtic calculat a partir de l'aproximació de la cadena hiper enllaçada. Es mostra que s'aconsegueix un bon ajust quantitatiu amb les dades de simulacions de dinàmica molecular.

D'igual manera, s'observa poca discrepància entre les característiques dinàmiques del plasma calculades a través dels factors d'estructura estàtica, enfront dels obtinguts per altres mètodes de càlcul d'aqueix factor d'estructura estàtica, com són l'aproximació de cadena hiper enllaçada (HNC, Hiper-NettedChain), la HNC modificada (MHNC, Modified Hiper-Netted Chain) i la HNC modificada variacionalmente (VMHNC, Variational Modified Hiper-Netted Chain). Aquesta estabilitat implica la robustesa de l'enfocament que es presenta.

Així mateix, s'analitzen les possibilitats d'abandonar l'aproximació estàtica NPF. 


\section{ACKNOWLEDGEMENTS}

It would be impossible to write and present this thesis without the support and assistance of many people, who surrounded me during all these years.

Firstly, I wish to thank my advisors Profs. Yuriy V. Arkhipov and Igor M. Tkachenko Gorski. I feel blessed to have such advisors, with whom I could contact on any question at any time and obtain qualified answers.

I owe my deepest gratitude to Askar Davletov, Saule Syzganbayeva and Asel Ashikbayeva, who supported me during all these years. I would like to thank Abdiadil Askaruly for the encouraging discussions and Khajimurat Santybayev for his help in difficult technical situations.

I thank Dr. Z. Donkó and his team, who provided me data from simulations on dynamic structure factors of one-component classical plasmas that were used in this thesis for comparison with the obtained results and for his advices about my work that he gave during our correspondence.

It is a pleasure to thank the family of Prof. Igor M. Tkachenko Gorski for their support when I really needed it and for their hospitality during my visits to Valencia.

Finally, I thank my parents and my close relatives for supporting me throughout all my studies at the University. 
1 Introduction 10

1.1 Motivation

1.2 Actuality

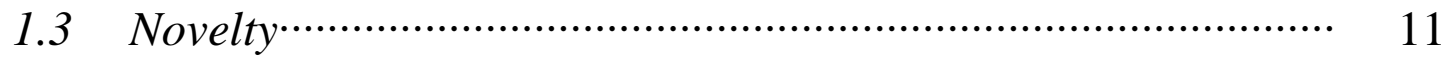

$1.4 \quad$ Thesis structure

2 Method of moments. The mathematical background 13

2.1 Nevanlinna (response) functions and their mathematical 13 properties

2.2 The classical Hamburger problem of moments ……………....... 14

2.3 Orthogonal polynomials and the Nevanlinna formula ………... 17

2.4 Canonical and degenerate solutions of a solvable truncated 21 Hamburger moment problem

2.5 Non-canonical solutions of a truncated Hamburger problem. 25 Application of the Nevanlinna formula

3 Application of the classical method of moments to the 30 investigation of dynamical properties of strongly coupled plasmas

4 Solution of physical problems by the self-consistent method of 40 moments

4.1 Dynamic structure factors …………………...................... 40

$4.2 \quad$ Dispersion equation …………………………………….... 53

4.3 Dynamic local field corrections …………………………..... 58

5 Analysis of schemes for the calculation of plasma static 72 characteristics

6 Nevanlinna parameter function investigation $\quad 79$

7 Conclusions $\quad 86$

$\begin{array}{lll}8 & \text { Appendix A } & 87\end{array}$

$\begin{array}{ll}\text { Bibliography } & 90\end{array}$ 


\begin{tabular}{|c|c|c|}
\hline 3.1 & $\begin{array}{l}\text { The COCP Shannon entropy as a function of the Nevanlinna } \\
\text { parameter } h(q) \text { for different wavenumber values }\end{array}$ & 38 \\
\hline 4.1 & $\begin{array}{l}\text { Irison of COCP DSFs, calculated as eq. (4.7) (lines) } \\
\text { ed from MD simulations [32] (lines with symbols). }\end{array}$ & 43 \\
\hline 4.2 & $\begin{array}{l}\text { The comparison of COCP DSFs, calculated as eq. (4.7) (lines) } \\
\text { and obtained from MD simulations [32] (lines with symbols). }\end{array}$ & 44 \\
\hline 4.3 & $\begin{array}{l}\text { The comparison of COCP DSFs, calculated as eq. (4.7) (lines) } \\
\text { and obtained from MD simulations [32] (lines with symbols). }\end{array}$ & 45 \\
\hline 4.4 & $\begin{array}{l}\text { The comparison of COCP DSFs, calculated as eq. (4.7) (lines) } \\
\text { and obtained from MD simulations [32] (lines with symbols). }\end{array}$ & 46 \\
\hline 4.5 & $\begin{array}{l}\text { as eq. (4.7) (solid } \\
\text { (squares). }\end{array}$ & 47 \\
\hline 4.6 & $\begin{array}{l}\text { as eq. (4.7) (lines) } \\
\text { s with symbols). }\end{array}$ & 48 \\
\hline 4.7 & $\begin{array}{l}\text { as eq. (4. } \\
\text { s with sym }\end{array}$ & 49 \\
\hline 4.8 & $\begin{array}{l}\text { The cc } \\
\text { and ob }\end{array}$ & 50 \\
\hline 4.9 & nes) & 51 \\
\hline 4.10 & The & 52 \\
\hline 4.11 & Dis & 54 \\
\hline 12 & Dispers & 55 \\
\hline 4 & DIspeIsic & 56 \\
\hline 4.14 & ared & 5 \\
\hline 4.15 & on (4.14) for the dynamic local field & 61 \\
\hline 4.16 & $\begin{array}{l}\text { Imaginary part of the expression (4.14) for the dynamic local } \\
\text { field correction. }\end{array}$ & 62 \\
\hline 4.17 & $\begin{array}{l}\text { Real part of the expression (4.14) for the dynamic local field } \\
\text { correction. }\end{array}$ & 6 \\
\hline 4.18 & $\begin{array}{l}\text { part of the expression (4.14) for the dynamic local } \\
\text { ction. }\end{array}$ & 62 \\
\hline 4. & $\begin{array}{l}\text { Real part of the expression (4.14) for the dynamic local field } \\
\text { correction. }\end{array}$ & $0=$ \\
\hline
\end{tabular}




\begin{tabular}{|l|l|c|}
\hline 4.20 & $\begin{array}{l}\text { Imaginary part of the expression (4.14) for the dynamic local } \\
\text { field correction. }\end{array}$ & 66 \\
\hline 4.21 & $\begin{array}{l}\text { Real part of the expression (4.14) for the dynamic local field } \\
\text { correction. }\end{array}$ & 67 \\
\hline 4.22 & $\begin{array}{l}\text { Imaginary part of the expression (4.14) for the dynamic local } \\
\text { field correction. }\end{array}$ & 68 \\
\hline 4.23 & $\begin{array}{l}\text { Dynamic structure factor (4.7) (lines), normalized to the shifted } \\
\text { maxima values in COCPs. }\end{array}$ & 69 \\
\hline 5.1 & $\begin{array}{l}\text { Static and dynamic structure factors in COCPs, compared to the } \\
\text { MD results (dots) [32]. }\end{array}$ & 73 \\
\hline 5.2 & $\begin{array}{l}\text { Static and dynamic structure factors in COCPs, compared to the } \\
\text { MD results (dots) [32]. }\end{array}$ & 74 \\
\hline 5.3 & $\begin{array}{l}\text { Static and dynamic structure factors in YOCPs, compared to the } \\
\text { MD results (dots) [32]. }\end{array}$ & 75 \\
\hline 5.4 & $\begin{array}{l}\text { Static and dynamic structure factors in YOCPs, compared to the } \\
\text { MD results (dots) [32]. }\end{array}$ & 76 \\
\hline 5.5 & The discrimination parameters $\theta(q)$ and $b(q)$ for COCP. & 77 \\
\hline 6.1 & $\begin{array}{l}\text { Dynamic structure factor fot the COCP presenting the method of } \\
\text { moments (MM) data vs. the MD data (dots) [32]. }\end{array}$ & 82 \\
\hline 6.2 & $\begin{array}{l}\text { Dynamic structure factor fot the COCP presenting the method of } \\
\text { moments (MM) data vs. the MD data (dots) [32]. }\end{array}$ & 83 \\
\hline 6.3 & $\begin{array}{l}\text { Dynamic structure factor fot the COCP presenting the method of } \\
\text { moments (MM) data vs. the MD data (dots) [32]. }\end{array}$ & 84 \\
\hline 6.4 & $\begin{array}{l}\text { Dynamic structure factor fot the COCP presenting the method of } \\
\text { moments (MM) data vs. the MD data (dots) [32]. }\end{array}$ & 85 \\
\hline
\end{tabular}




\section{INTRODUCTION}

1.1. Motivation. One of the challenges of the contemporary applied sciences is the scarceness of sources of energy for the growing necessities of the humanity. The answer, as of today, is the controlled nuclear fusion, which employs as the fuel the hydrogen and its isotopes, the most abundant element in the Universe. Besides, contrary to the fission, the fusion is much less dangerous from the point of view of contamination and explosiveness.

The problem is that the physical conditions (temperature and density) of the working body of the future inertial fusion reactors, where a target made of the isotopes of hydrogen (deuterium and tritium) which exist in Nature, is strongly compressed by external laser radiation or heavy-ion jets to the densities up to 5 order higher than that of metals. Certainly, under such extreme conditions traditional physical theories based on expansions in some small parameters, fail and to diagnose and control the processes in the working body, one needs alternative non-perturbative physical theories.

This is exactly the aim of the present work where we have employed the classical method of moments, namely, the Hamburger problem of moments that possesses two infinite families of solutions, complemented by some physical considerations. Precisely, a specific non-canonical solution is used to construct a physically motivated unique solution of a truncated problem of reconstruction of a Nevanlinna (called in Statistical Physics, response) function, whose power moments are known in Statistical Physics as the sum rules. Such a self-consistent version of the moment approach has led to the quantitative description of important dynamical and optical properties of model physical systems known as the one-component plasmas under the conditions similar to those of future fusion devices. There are four advantages of the present theoretical construction: (i) all dynamic characteristics are expressed in terms of the static ones which are much easier 
to compute; (ii) the approach admits an expansion to partly degenerate systems (like the electron gas), (iii) other exact relations and mathematical methods like, e.g., the Shannon entropy maximization are also employed, (iv) all calculations do not require very high numerical performance and can be carried out with a medium-quality laptop, i.e., the method permits to diagnose the body of the fusion device in the on-line regime.

1.2. Actuality. The method of moments occupies a special place among the theoretical methods dedicated to the study of systems with Coulomb interaction between particles. Its essence lies in the fact that the system linear response function is parameterized as a fractional-linear transformation of a parameter function with certain mathematical properties. The coefficients of the transformation are the orthogonal polynomials calculated from the first converging power moments of the imaginary part of the response function. The moments can be calculated independently, within the Kubo linear reaction theory. Our self-consistent approach allows to reconstruct the dynamic characteristics of the physical system that we study in terms of the static ones.

1.3. Novelty. Based on the moments method, a theoretical approach is constructed that allows to calculate the electrodynamic properties of plasmas using its static characterisitcs.

1.4. Thesis structure. In the first section, in the Introduction, the applicability of the chosen research topic, the aims, the method are generally motivated. The second section presents the mathematical background of the method of moments. The third section gives the information about the applicability of method of moments to classical one-component plasmas. The fourth chapter provides description of the calculation of the dynamic characteristics of classical one-component plasmas. The fifth section deals with the analysis of schemes for the calculation of static structure factor and also contains some additional results. The sixth section presents several ways 
for the calculation of Nevanlinna parameter function. The seventh section contains the Conclusions. In the Appendix there is the list of publications and international conferences. The thesis is ended by the Bibliography. 


\section{METHOD OF MOMENTS. THE MATHEMATICAL BACKGROUND.}

\subsection{Nevanlinna (response) functions and their mathematical properties.}

Definition 1 The Nevanlinna class of functions $\mathfrak{R}$ :

A function $F(z) \in \mathfrak{R}$ if

1. $F(z)$ is analytic in $\operatorname{Im} z>0$;

2. $\operatorname{Im} F(z) \geq 0$ in $\operatorname{Im} z>0$.

Definition 2 Let $t \in \mathbb{R}$ be a random variable with a distribution function $\sigma(t)$. If

$$
\sigma(t)=\int_{-\infty}^{t} f(s) d s,
$$

the function $f(t)$ is called the probability density function, p.d.f. Since $\sigma(t)$ is, by definition, a non-decreasing function, $f(t) \geq 0$ for any real $t$.

Claim 3. The Nevanlinna functions are determined by the Riesz - Herglotz transform:

$$
F(z)=a z+b+\int_{-\infty}^{\infty}\left(\frac{1}{t-z}-\frac{t}{1+t^{2}}\right) d g(t),
$$

where $\{a, b\} \in \mathbb{R}, a \geq 0$ and $g(t)$ is a non-decreasing bounded function (distribution) such that

$$
\int_{-\infty}^{\infty} \frac{d g(t)}{1+t^{2}}<\infty
$$

Claim 4 Notice that we can always choose the function $g(t)$ so that $a=0$ and $b$ was equal to

$$
b=\int_{-\infty}^{\infty} \frac{t d g(t)}{1+t^{2}} .
$$


Definition 5 The class of functions $\mathfrak{R}_{0}$ :

A function $G(z) \in \mathfrak{R}_{0}$ if $G(z) \in \mathfrak{R}$ and

$$
\lim _{z \rightarrow \infty} \frac{G(z)}{z}=0, \operatorname{Im} z>0,
$$

so that for such functions from (2.3) we have:

$$
G(z)=\int_{-\infty}^{\infty} \frac{d g(t)}{t-z}+i h, h>0,
$$

where the non-negative parameter $h$ does not depend on $z$, but might depend on other parameters, e.g., in Physics, on the wavenumber.

\subsection{The classical Hamburger problem of moments}

Definition 6 The real numbers

$$
\mu_{m}=\int_{-\infty}^{\infty} t^{m} d \sigma(t), m=0,1,2, \ldots
$$

are the (power) moments of the distribution $\sigma(t)$. If the distribution $\sigma(t)$ is differentiable and $f(t)=\sigma^{\prime}(t)$ is symmetric, all odd-order moments (2.5) vanish.

Let us summarize some notions and results of the classical theory of moments [1-3]. The Hamburger problem is formulated in the following way. Problem 7 Given a set of real numbers $\left\{\mu_{0}, \mu_{1}, \mu_{2}, \ldots\right\}$, find all distributions $\sigma(t)$ such that

$$
\int_{-\infty}^{\infty} t^{m} d \sigma(t)=\mu_{m}, m=0,1,2, \ldots
$$

The Hamburger moment problem is solvable, i.e., there exists at least one distribution (p.d.f.) which satisfies (2.6), if and only if the given set of 
numbers $\left\{\mu_{m}\right\}_{m=0}^{\infty}$ is non-negative, i.e., if the Hankel matrix $\left(\mu_{m+n}\right)_{m, n=0}^{\infty} \geq 0$. If the problem is solvable, it can have a unique solution (a determinate problem) or an infinite number of solutions (an indeterminate problem).

Definition 8. Notice that if $\sigma(t<0) \equiv$ const (i.e., if $f(t<0) \equiv 0$ ), we have the Stieltjes moment problem, and if $\sigma(t) \equiv$ const $(f(t) \equiv 0)$ for $t<a, t>b, a, b \in$ $\mathbb{R}$, we deal with the Hausdorff problem finite interval moment problem.

Theorem 9 [4] A Hamburger moment problem (2.6) is solvable if

$$
\Delta_{m}=\operatorname{det}\left(\mu_{i+j}\right)_{i, j=0}^{m} \geq 0, m=0,1,2, \ldots
$$

The problem has an infinite number of solutions if and only if

$$
\Delta_{m}=\operatorname{det}\left(\mu_{i+j}\right)_{i, j=0}^{m}>0, m=0,1,2, \ldots
$$

The problem (2.6) is determinate if and only if

$$
\Delta_{0}>0, \ldots, \Delta_{k}>0, \Delta_{k+1}=\Delta_{k+2}=\ldots=0 .
$$

Claim 10. The set of solutions of an indeterminate problem is in a one-to one correspondence with a certain subset of the class of Nevanlinna functions [1]; this correspondence is described by the Nevanlinna formula, see below.

Claim 11. A truncated Hamburger moment problem [5], i.e., a moment problem with a finite set of given numbers, i.e., $\left\{\mu_{m}\right\}_{m=0}^{2 v}, v=0,1,2$ is solvable if the Hankel matrix $\left(\mu_{m+n}\right)_{m, n=0}^{v}>0$, [6], see also [7] and [8]. In the degenerate case of a singular Hankel matrix $\left(\mu_{m+n}\right)_{m, n=0}^{v}$ the problem of moments (under some special conditions established in [9]) has a unique solution.

Theorem 12 [10] A sufficient condition that the Hamburger moment problem (2.6) be determinate is that (Carleman's criterion) 


$$
\sum_{m=1}^{\infty} \mu_{2 m}^{-1 / 2 m}=\infty
$$

Example 13. The p.d.f

$$
f_{\alpha}(t ; \gamma)=\frac{\alpha \gamma^{1 / \alpha}}{2 \Gamma\left(\frac{1}{\alpha}\right)} \exp \left(-\gamma|t|^{\alpha}\right), \alpha, \gamma>0
$$

where $\Gamma(z)$ is the Euler $\Gamma$ function, has an infinite number of moments for any positive $\alpha$ :

$$
\begin{gathered}
\mu_{2 m}(\alpha ; \gamma)=\int_{-\infty}^{\infty} t^{2 m} f_{\alpha}(t) d t=\frac{\Gamma\left(\frac{2 m+1}{\alpha}\right)}{\gamma^{2 m / \alpha} \Gamma\left(\frac{1}{\alpha}\right)}, \\
\mu_{2 m+1}(\alpha ; \gamma)=0, m=0,1,2, \ldots
\end{gathered}
$$

but the Hamburger moment problem for the set of numbers

$$
\left\{1,0, \frac{\Gamma\left(\frac{3}{\alpha}\right)}{\gamma^{2 / \alpha} \Gamma\left(\frac{1}{\alpha}\right)}, 0, \frac{\Gamma\left(\frac{5}{\alpha}\right)}{\gamma^{4 / \alpha} \Gamma\left(\frac{1}{\alpha}\right)}, 0, \ldots\right\},
$$

has, as it stems from the Carleman criterion, a unique solution, which is the p.d.f. (1.7), if $\alpha>1$, in particular the Gaussian density $f_{2}\left(t ; \frac{1}{2 a^{2}}\right), a>0$, and an infinite number of solutions if $\alpha \leq 1$. In this latter case, all solutions of the moment problem are described by the Nevanlinna formula ([2]), see below.

Other examples of sets $\left\{\mu_{m}\right\}_{m=0}^{\infty}$ which generate indeterminate moment problems are provided in [3].

In (solvable) problems where we already have at least one p.d.f. with a set of moments, like the problems we are interested in here, the only 
question which arises is the one of uniqueness of the solution of the problem of reconstruction of a (one-dimensional) p.d.f. by its power moments, $\left\{\mu_{m}\right\}_{m=0}^{v}$.

\subsection{Orthogonal polynomials and the Nevanlinna formula}

Theorem 14. (Nevanlinna) There is a one-to-one correspondence between all solutions of the Hamburger problem (2.6), or all complex Nevanlinna functions

$$
\varphi(z)=\int_{-\infty}^{\infty} \frac{d \sigma(t)}{t-z}
$$

and all Nevanlinna functions $R(z) \in \mathfrak{R}_{0}$ such that

$$
\varphi(z)=\int_{-\infty}^{\infty} \frac{d \sigma(t)}{t-z}=-\frac{E_{n+1}(z)+R(z) E_{n}(z)}{D_{n+1}(z)+R(z) D_{n}(z)} .
$$

This last formula is called the Nevanlinna formula.

Definition 15 Here $\left\{D_{l}(z)\right\}_{l=0}^{\infty}$ are orthonormalized polynomials with respect to the measure $d \sigma[3]$ :

$$
\int_{-\infty}^{\infty} D_{n}(t) D_{m}(t) d \sigma(t)=\delta_{n m}, n, m=0,1, \ldots
$$

and $E_{n}(z)$ are their conjugate polynomials:

$$
E_{n}(z)=\int_{-\infty}^{\infty} \frac{D_{n}(z)-D_{n}(t)}{z-t} d \sigma(t)
$$

Precisely, we can write 


$$
\begin{gathered}
D_{0}(t)=\frac{1}{\sqrt{\mu_{0}}}, \Delta_{-1}=1, \Delta_{0}=\mu_{0}, \\
D_{l}(t)=\frac{1}{\sqrt{\Delta_{l} \Delta_{l-1}}} \operatorname{det}\left[\begin{array}{cccc}
\mu_{0} & \cdots & \mu_{l-1} & 1 \\
\mu_{1} & \cdots & \mu_{l} & t \\
\vdots & \vdots & \vdots & \vdots \\
\mu_{l} & \cdots & \mu_{2 l-1} & t^{l}
\end{array}\right], \\
\Delta_{l}=\operatorname{det}\left[\begin{array}{ccc}
\mu_{0} & \cdots & \mu_{l} \\
\vdots & \vdots & \vdots \\
\mu_{l} & \cdots & \mu_{2 l}
\end{array}\right], l=1,2, \cdots
\end{gathered}
$$

Let us point out the properties of these orthonormalized polynomials.

Claim 16. It can be easily seen that both sets of polynomials do not depend on the distribution we seek; they are determined by the moments only:

$$
\begin{gathered}
D_{0}(z)=\frac{1}{\sqrt{\mu_{0}}}, D_{1}(z)=\frac{1}{\sqrt{\mu_{0}}} \frac{z-a_{0}}{b_{0}}, \\
D_{2}(z)=\frac{\left(\mu_{0} \mu_{2}-\mu_{1}^{2}\right) z^{2}+z\left(\mu_{1} \mu_{2}-\mu_{3} \mu_{0}\right)+\left(\mu_{3} \mu_{1}-\mu_{2}^{2}\right)}{\sqrt{\left(\mu_{0} \mu_{2}-\mu_{1}^{2}\right) \Delta_{2}}}, \ldots . \\
E_{0}(z)=0, E_{1}(z)=\frac{\sqrt{\mu_{0}}}{b_{0}}, \\
E_{2}(z)=\frac{\mu_{0}\left(\mu_{0} \mu_{2}-\mu_{1}^{2}\right) z+\left(\mu_{0} \mu_{2}-\mu_{1}^{2}\right) \mu_{1}+\mu_{0}\left(\mu_{1} \mu_{2}-\mu_{0} \mu_{3}\right)}{\sqrt{\left(\mu_{0} \mu_{2}-\mu_{1}^{2}\right) \Delta_{2}}}
\end{gathered}
$$$$
, \ldots
$$ 
In addition:

1. The zeros of the polynomials $D_{l}(t)$ and $E_{l}(t), l \in \mathbb{N}$, are all real;

2. The zeros of the polynomials $D_{l}(t)$ and $D_{l-l}(t), l \in \mathbb{N}$, alternate.

3. The zeros of the polynomials $D_{l}(t)$ and $E_{l}(t), l \in \mathbb{N}$, alternate;

4. The polynomials $D_{l}(t)$ and $E_{l}(t), l \in \mathbb{N}$, can be expressed in terms of each other:

$$
\begin{aligned}
& z D_{l}(z)=b_{l-1} D_{l-1}(z)+a_{l} D_{l}(z)+b_{l} D_{l+1}(z), l=1,2, \ldots \\
& z E_{l}(z)=b_{l-1} E_{l-1}(z)+a_{l} E_{l}(z)+b_{l} E_{l+1}(z), \quad l=1,2, \ldots
\end{aligned}
$$

where the coefficients can be written as

$$
\begin{gathered}
a_{l}=a_{l, l}=\int_{-\infty}^{\infty} t D_{l}(t) D_{l}(t) d \sigma(t), \\
b_{l}=a_{l, l+1}=\int_{-\infty}^{\infty} t D_{l}(t) D_{l+1}(t) d \sigma(t)=\frac{\sqrt{\Delta_{l-1} \Delta_{l+1}}}{\Delta_{l}}, l=1,2, \ldots
\end{gathered}
$$

5. They satisfy the Liouville-Ostrogradsky (or SchwarzChristoffel) formula:

$$
D_{l-1}(z) E_{l}(z)-D_{l}(z) E_{l-1}(z)=\frac{1}{b_{l-1}}=\frac{\Delta_{l-1}}{\sqrt{\Delta_{l-2} \Delta_{l}}}, l=2,3, \ldots .
$$

Claim 17. The latter relation permits to define these polynomials in the recurrent way. Indeed, since 


$$
\begin{gathered}
D_{0}(z)=\frac{1}{\sqrt{\mu_{0}}}, \quad D_{1}(z)=\frac{1}{\sqrt{\mu_{0}}} \frac{z-a_{0}}{b_{0}}, \\
E_{0}(z)=0, E_{1}(z)=\frac{\sqrt{\mu_{0}}}{b_{0}},
\end{gathered}
$$

we have that

$$
\begin{gathered}
D_{2}(z)=\frac{\left(\mu_{0} \mu_{2}-\mu_{1}^{2}\right) z^{2}+z\left(\mu_{1} \mu_{2}-\mu_{3} \mu_{0}\right)+\left(\mu_{3} \mu_{1}-\mu_{2}^{2}\right)}{\sqrt{\left(\mu_{0} \mu_{2}-\mu_{1}^{2}\right) \Delta_{2}}}, \\
E_{2}(z)=\frac{\mu_{0}\left(\mu_{0} \mu_{2}-\mu_{1}^{2}\right) z+\left(\mu_{0} \mu_{2}-\mu_{1}^{2}\right) \mu_{1}+\mu_{0}\left(\mu_{1} \mu_{2}-\mu_{0} \mu_{3}\right)}{\sqrt{\left(\mu_{0} \mu_{2}-\mu_{1}^{2}\right) \Delta_{2}}},
\end{gathered}
$$

and so on. This procedure can be easily programmed.

Claim 18. It can be easily checked that the polynomials $D_{\ell}(z), \ell=0,1,2$ are all normalized to unity and mutually orthogonal.

Claim 19. The set of orthogonal (but not normalized) polynomials $\left\{\mathrm{D}_{\ell}(t)\right\}_{\ell=0}^{\infty}$ can be constructed from the canonical basis of the Hilbert vector space of polynomials,

$$
\left\{1, t, t^{2}, \ldots\right\}
$$

but with the scalar product and the norm defined as

$$
\langle f, g\rangle=\int_{-\infty}^{\infty} f(t) \overline{\mathrm{g}(t)} d \sigma(t),\|f\|=\sqrt{\langle f, f\rangle},
$$

by means of the standard Gram-Schmidt procedure. Then,

$$
D_{0}(t)=1, D_{1}(t)=t-\frac{\mu_{1}}{\mu_{0}}, \quad D_{2}(t)=t^{2}-t \frac{\mu_{1} \mu_{2}-\mu_{0} \mu_{3}}{\mu_{1}^{2}-\mu_{0} \mu_{2}}+\frac{\mu_{2}^{2}-\mu_{3} \mu_{1}}{\mu_{1}^{2}-\mu_{0} \mu_{2}},
$$




$$
\begin{gathered}
D_{3}(t)=t\left(t^{2}+A t+B\right), E_{0}(t)=0, E_{1}(t)=\mu_{0}, \\
E_{2}(t)=\mu_{0}\left(t-\frac{\mu_{1} \mu_{2}-\mu_{0} \mu_{3}}{\mu_{1}^{2}-\mu_{0} \mu_{2}}\right)+\mu_{1}, \\
E_{3}(t)=\mu_{0}\left(t^{2}+t\left(A+\frac{\mu_{1}}{\mu_{0}}\right)+\left(\frac{\mu_{2}}{\mu_{0}}+A \frac{\mu_{1}}{\mu_{0}}+B\right)\right),
\end{gathered}
$$

where the coefficients $A$ and $B$ have the form

$$
\begin{gathered}
A=\frac{\mu_{1}\left(\mu_{3}^{2}+\mu_{2} \mu_{4}\right)-\mu_{3}\left(\mu_{2}^{2}+\mu_{0} \mu_{4}\right)-\mu_{5}\left(\mu_{1}^{2}-\mu_{0} \mu_{2}\right)}{\mu_{2}\left(\mu_{2}^{2}-\mu_{0} \mu_{4}\right)+\mu_{3}\left(\mu_{0} \mu_{3}-\mu_{1} \mu_{2}\right)+\mu_{1}\left(\mu_{4} \mu_{1}-\mu_{2} \mu_{3}\right)}, \\
B=\frac{\mu_{5}\left(\mu_{1} \mu_{2}-\mu_{0} \mu_{3}\right)-\mu_{3}\left(\mu_{1} \mu_{4}-\mu_{2} \mu_{3}\right)-\mu_{4}\left(\mu_{2}^{2}-\mu_{0} \mu_{4}\right)}{\mu_{2}\left(\mu_{2}^{2}-\mu_{0} \mu_{4}\right)+\mu_{3}\left(\mu_{0} \mu_{3}-\mu_{1} \mu_{2}\right)+\mu_{1}\left(\mu_{4} \mu_{1}-\mu_{2} \mu_{3}\right)} .
\end{gathered}
$$

An important observation mentioned above can be deduced from the expressions (1.14) and (1.20): both sets of orthogonal polynomials do not depend on the distribution we seek; they are determined by the moments only. In other words, these polynomials are known as soon as the moments are.

\subsection{Canonical and degenerate solutions of a solvable truncated Hamburger moment problem}

Claim 20. It is clear that, at least, due to numerical and measurement problems, we never know a large number of moments. Besides, as we will see, in certain physically important problems, this number is limited by physical phenomena.

In order to satisfy the moment conditions

$$
\mu_{m}=\int_{-\infty}^{\infty} t^{m} d \sigma(t)=\int_{-\infty}^{\infty} t^{m} f(t) d t, m=0,1,2, \ldots, 2 v, v=0,1,2, \ldots
$$


one can first consider a step-like distribution

$$
d \sigma(t)=\sum_{j=0}^{2 v} m_{j} \delta\left(t-t_{j}\right) d t
$$

with the density which actually consists of $2 v+1$ point masses located at some distinct points of the real axis $\left\{t_{j}\right\}_{j=0}^{2 \nu}$. This is the so called canonical solution of the problem. Then the assumption (2.22) can be substituted into the conditions (2.21) and the masses $\left\{m_{j}\right\}_{j=0}^{2 \nu}$ can be obtained directly from the system with the determinant which is the Van der Monde determinant of an arbitrary set of distinct numbers $\left\{t_{j}\right\}_{j=0}^{2 \nu}$ :

$$
\left[\begin{array}{cccc}
1 & 1 & \cdots & 1 \\
t_{0} & t_{1} & \cdots & t_{2 v} \\
\vdots & \vdots & \ddots & \vdots \\
t_{0}^{2 v} & t_{1}^{2 v} & \cdots & t_{2 v}^{2 v}
\end{array}\right]\left[\begin{array}{c}
m_{0} \\
m_{1} \\
\vdots \\
m_{2 v}
\end{array}\right]=\left[\begin{array}{c}
\mu_{0} \\
\mu_{1} \\
\vdots \\
\mu_{2 v}
\end{array}\right]
$$

In other words, we obtain an infinite number of canonical solutions parametrized by the latter set of points of the real axis.

Example 21. Gaussian distribution $\exp \left(-t^{2}\right)$. Consider a truncated problem generated by the moments

$$
\begin{gathered}
\mu_{m}=\int_{-\infty}^{\infty} t^{m} \exp \left(-t^{2}\right) d t, m=0,1,2, \ldots, 2 v . \\
\mu_{0}=\sqrt{\pi}, \mu_{1}=0, \mu_{2}=\frac{\sqrt{\pi}}{2} .
\end{gathered}
$$


Then the system (2.23) becomes:

$$
\left[\begin{array}{ccc}
1 & 1 & 1 \\
t_{0} & t_{1} & t_{2} \\
t_{0}^{2} & t_{1}^{2} & t_{2}^{2}
\end{array}\right]\left[\begin{array}{l}
m_{0} \\
m_{1} \\
m_{2}
\end{array}\right]=\left[\begin{array}{c}
\sqrt{\pi} \\
0 \\
\sqrt{\pi} / 2
\end{array}\right] .
$$

Its solution is just:

$$
\left(\begin{array}{l}
m_{0} \\
m_{1} \\
m_{2}
\end{array}\right)=\frac{\sqrt{\pi}}{2}\left(\begin{array}{l}
\frac{\left(2 x_{1} x_{2}+1\right)}{\left(x_{2}-x_{0}\right)\left(x_{1}-x_{0}\right)} \\
\frac{\left(2 x_{0} x_{2}+1\right)}{\left(x_{2}-x_{1}\right)\left(x_{0}-x_{1}\right)} \\
\frac{\left(2 x_{0} x_{1}+1\right)}{\left(x_{1}-x_{2}\right)\left(x_{0}-x_{2}\right)}
\end{array}\right) .
$$

Claim 22. Nevertheless, for the moment set $\left\{\mu_{0}, 0, \mu_{2}\right\}$, there exists the following canonical solution of the moment problem where

$$
\begin{gathered}
\int_{-\infty}^{\infty} t^{m} f(t) d t=\mu_{m}, m=0,1,2 . \\
f(t)=\frac{\mu_{0}}{2}[\delta(t-\xi)+\delta(t+\xi)], \quad \xi^{2}=\frac{\mu_{2}}{\mu_{0}} .
\end{gathered}
$$

Claim 23. While, for the moment set $\left\{\mu_{0}, 0, \mu_{2}, 0, \mu_{4}\right\}$, there exists the following canonical solution of the moment problem

$$
\begin{gathered}
\int_{-\infty}^{\infty} t^{m} f(t) d t=\mu_{m}, m=0,1,2,3,4, \\
f(t)=\mu_{0}\left\{\left(1-\frac{\xi_{1}^{2}}{\xi_{2}^{2}}\right) \delta(t)+\frac{\xi_{1}^{2}}{2 \xi_{2}^{2}}\left[\delta\left(t-\xi_{2}\right)+\delta\left(t+\xi_{2}\right)\right]\right\},
\end{gathered}
$$

where we can write 


$$
\xi_{1}^{2}=\frac{\mu_{2}}{\mu_{0}}, \quad \xi_{2}^{2}=\frac{\mu_{4}}{\mu_{2}}
$$

This solution will be interpreted later, dedicated to the investigation of onecomponent plasmas. The positivity of the central feature intensity follows from the Cauchy-Schwarz inequality.

Example 24. Degenerate case. Consider now a degenerate truncated problem generated by the moments

$$
\mu_{0}=1, \mu_{1}=\sqrt{2}, \mu_{2}=2,
$$

whose Hankel matrix

$$
H_{1}=\left[\begin{array}{cc}
1 & \sqrt{2} \\
\sqrt{2} & 2
\end{array}\right]
$$

is obviously singular ( $\left.\operatorname{det} H_{1}=0\right)$. In this case the solution of the problem is unique, it can be found in the following way. Find the null-space basis of the matrix $H_{1}$, in our case it is a vector $\left[\begin{array}{c}-\sqrt{2} \\ 1\end{array}\right]:=\left[\begin{array}{c}\xi_{0} \\ \xi_{1}\end{array}\right]$ with $\xi_{1} \neq 0$, construct the polynomial

$$
p(t)=\xi_{1} t+\xi_{0},
$$

calculate its zeros (in our case we have only one zero $t_{0}=\sqrt{2}$ ), these are the locations $\left\{t_{i}\right\}_{i=1}^{\nu}$ of the masses in the degenerate solution

$$
d \sigma(t)=\sum_{i=0}^{2 v} m_{j} \delta\left(t-t_{i}\right) d t
$$

and determine the corresponding masses from the moment conditions (2.21). Particularly, for the moments (1.24) we have 


$$
d \sigma(t)=\delta(t-\sqrt{2}) d t
$$

which automatically satisfies the conditions

$$
\mu_{0}=1, \mu_{1}=\sqrt{2}, \mu_{2}=2 \text {. }
$$

Claim 25. Certainly, in physical problems we are basically interested in noncanonical, continuous solutions Nevertheless, some physical interpretation of the canonical solutions will be discussed as well. To show how the moment method works in this case, let us consider dynamical properties of the intrinsically classical one - and two - component completely ionized hydrogen - like plasmas in thermal equilibrium.

\subsection{Non-canonical solutions of a truncated Hamburger problem.} Application of the Nevanlinna formula

In physical problems we deal with further, we are interested in continuous solutions of truncated Hamburger problems generated by positive sets of power moments

$$
\left\{\mu_{0}, \mu_{1}, \mu_{2}, \ldots, \mu_{2 v-1}, \mu_{2 v}\right\}, v=0,1,2, \ldots,
$$

basically, with $v=2$ and with the so called immaterial elements $\mu_{2 v+1}$ and $\mu_{2 v+2}$. Let us see how the Nevanlinna formula in this case provides a continuous, non-canonical, solution of the problem: construct the probability density function $f(t)$ such that

$$
\mu_{l}=\int_{-\infty}^{\infty} t^{l} f(t) d t, l=0,1,2, \ldots, 2 v, \quad v=0,1,2, \ldots .
$$


The Nevanlinna formula in this case takes the following form:

$$
\varphi(z)=\int_{-\infty}^{\infty} \frac{f(t)}{t-z} d t=-\frac{E_{v+1}(z)+R_{v}(z) E_{v}(z)}{D_{v+1}(z)+R_{v}(z) D_{v}(z)} .
$$

Claim 26. Observe that the Nevanlinna parameter function $R_{v}(z) \in \mathfrak{R}_{0}$ effectively depends on the number of moments involved. Nevertheless, the asymptotic expansion of the Cauchy transform of the density in question will satisfy the moment conditions (2.25) independently of our choice of this parameter function.

Proof. Indeed, along any ray within the upper half-plane $\operatorname{Im} z>0$,

$$
\begin{gathered}
\varphi(z \rightarrow \infty)=-\frac{1}{z} \int_{-\infty}^{\infty} \frac{f(x)}{1-\frac{x}{z}} d x \simeq \\
\underset{z \rightarrow \infty}{\simeq}-\frac{1}{z} \int_{-\infty}^{\infty} f(x)\left(\sum_{l=0}^{2 v}\left(\frac{x}{z}\right)^{l}+O\left(\frac{1}{z}\right)^{2 v+1}\right) d x= \\
=-\sum_{l=0}^{2 v} \frac{1}{z^{l+1}} \int_{-\infty}^{\infty} x^{l} f(x) d x+O\left(\frac{1}{z}\right)^{2 v+2}= \\
=-\sum_{l=0}^{2 v} \frac{\mu_{l}}{z^{l+1}}+O\left(\frac{1}{z}\right)^{2 v+2} .
\end{gathered}
$$

In other words, the contribution related to the Nevanlinna parameter function $R_{v}(z)$, due to the (2.3), will appear in the asymptotic expansion (2.27) only in the correction of excessive order $2 v+2$. Now, by definition, on the real axis $\operatorname{Im} z=0$,

$$
\varphi(t)=\operatorname{Im}\left(\lim _{\eta \downarrow 0} \int_{-\infty}^{\infty} \frac{f(s) d s}{s-t-i \eta}\right)=
$$




$$
\begin{gathered}
=\operatorname{Im}\left(P \cdot V \cdot \int_{-\infty}^{\infty} \frac{f(s) d s}{s-t}+\pi i f(t)\right)= \\
=\pi f(t)=-\operatorname{Im} \frac{E_{v+1}(t)+R_{v}(t) E_{v}(t)}{D_{v+1}(t)+R_{v}(t) D_{v}(t)},
\end{gathered}
$$

$P . V$ standing for the principal value of the integral. Let

$$
R(t)=\operatorname{Re} R(t)+i \operatorname{Im} R(t), \overline{R(t)}=\operatorname{Re} R(t)-i \operatorname{Im} R(t),
$$

and observe that, also by definitions (2.14) and (2.15), we have:

$$
D_{v+1}(t)=\frac{1}{\sqrt{\Delta_{v+1} \Delta_{v}}} \operatorname{det}\left[\begin{array}{ccccc}
\mu_{0} & \cdots & \mu_{v-1} & \mu_{v} & 1 \\
\mu_{1} & \cdots & \mu_{v} & \mu_{v+1} & t \\
\vdots & \cdots & \vdots & \vdots & \vdots \\
\mu_{v-1} & \cdots & \mu_{2 v-1} & \mu_{2 v} & t^{v} \\
\mu_{v} & \cdots & \mu_{2 v} & \mu_{2 v+1} & t^{v+1}
\end{array}\right],
$$

so that the algebraic minor, (subdeterminant) of the $D_{v+1}(t)$ polynomial leading term is just the Hankel determinant

$$
\Delta_{v}=\operatorname{det}\left[\begin{array}{cccc}
\mu_{0} & \cdots & \mu_{v-1} & \mu_{v} \\
\mu_{1} & \cdots & \mu_{v} & \mu_{v+1} \\
\vdots & \vdots & \vdots & \vdots \\
\mu_{v-1} & \cdots & \mu_{2 v-1} & \mu_{2 v}
\end{array}\right],
$$

Hence

$$
D_{v+1}(t)=\sqrt{\frac{\Delta_{v}}{\Delta_{v+1}}} \mathrm{P}_{v+1}(t), D_{v}(t)=\sqrt{\frac{\Delta_{v-1}}{\Delta_{v}}} \mathrm{P}_{v}(t),
$$

where $\left\{\mathrm{P}_{l}(t)\right\}_{l=0}^{v+1}$ are orthogonal monic polynomials with respect to the measure density $f(t)$, see the Claim 19 . Thus, due to the LiouvilleOstrogradsky equality, the "problem" is that the determinant $\Delta_{v+1}$ (see (2.28)) contains the "immaterial" moments $\mu_{2 v+1}$ and $\mu_{2 v+2}$, which we do not 
know. They might even diverge! This spurious contradiction is immediately resolved by taking into account the normalization of the orthonormalized polynomials $\{\mathrm{P}(t)\}_{l=0}^{v+1}$ : use instead the monic polynomials $\left\{\mathrm{P}_{l}(t)\right\}_{l=0}^{2 v}$ :

$$
\begin{aligned}
f(t) & =\frac{\Delta_{v}}{\pi \sqrt{\Delta_{v-1} \Delta_{v+1}}} \frac{\operatorname{Im} R_{v}(t)}{\left|P_{v+1}(t)+R_{v}(t) P_{v}(t)\right|^{2}}= \\
& =\frac{\Delta_{v}}{\pi \Delta_{v-1}} \frac{\operatorname{Im} Q_{v}(t)}{\left|P_{v+1}(t)+Q_{v}(t) P_{v}(t)\right|^{2}}>0
\end{aligned}
$$

where $Q_{v}(t)=R_{v}(t) \frac{\sqrt{\Delta_{v-1} \Delta_{v+1}}}{\Delta_{v}}$.

Notice that due to the positivity of the moment sequence (2.25), the Hankel determinants $\Delta_{v-1}$ and $\Delta_{v}$ are all strictly positive. Thus, the immaterial members of the moment sequence are eliminated due to the renormalization procedure. What matters for the physical applications is that the poles of the reconstructed density $f(z), \operatorname{Im} z<0$ are the roots of the "polynomial" equation

$$
\mathrm{P}_{v+1}(z)+Q_{v}(z) \mathrm{P}_{v}(z)=0
$$

which "starts" from $z^{v+1}$, i.e., if, in accordance with the $\Re_{0}$-version of the Riesz-Herglotz formula (2.4), we approximate the Nevanlinna parameter function (NPF) $q_{v}(z)$ by its static value

$$
Q_{v}(z)=Q_{v}(z=0)=i h
$$

equation (2.31) acquires the form of the genuine polynomial equation of the order $v+1$, which can be easily solved at least numerically. 
Generally speaking, the moment approach is originally based on the canonical or Nevanlinna's [11,12] non-canonical solutions of the (truncated) Hamburger moment problem consisting in the reconstruction of a non-negative nondecreasing distribution density by a finite number of its power moments.

The background of the moment approach is certainly purely mathematical with the specifics of a physical system involved only in the moments; in this sense the approach is model-free. The infinite set of canonical solutions is constructed by placing certain point "masses" at some points of the real axis. The values of the masses are determined by the moments while the location points can be chosen arbitrarily. Nevertheless, they can be specified by physical considerations.

On the other hand, the non-canonical solutions are parametrized by the Nevanlinna parameter function (NPF) of a certain mathematical class (see below), which, certainly, cannot be determined within the moment problem formalism. The simplest mathematically admissible approximation for the NPF is to substitute it by its static (purely imaginary) value. In the next chapter we will show, how one can use the method of moments for the strongly-coupled plasmas (SCP) investigation. 


\section{APPLICATION OF THE CLASSICAL METHOD OF MOMENTS TO THE INVESTIGATION OF DYNAMICAL PROPERTIES OF STRONGLY COUPLED PLASMAS}

Strongly coupled plasmas (SCPs) appear in various settings in nature (e.g., in dense astrophysical matter in white dwarfs and neutron stars [13]), as well as in the laboratory (in ultracold plasmas [14], electrolytes and charged stabilized colloids [15], laser-cooled ions in cryogenic traps [16], and dusty plasmas [17]). SCPs and warm dense matter are highly relevant model systems for inertial fusion devices [18]. The common property of SCPs is that the interparticle potential energy dominates over the thermal energy.

We deal with one component model (OCP) of SCPs. Two main types of potentials are of interest in OCPs. The Coulomb potential describes systems where the background of the oppositely charged species is not polarizable (e.g., a degenerate electron liquid embedding positive ions) systems with this property are further referred to as Coulomb OCPs (COCPs), while the Yukawa OCPs (YOCPs) are characterized by a screened-Coulomb (Debye, or Yukawa) potential between the "primary" species, where the screening is established by "secondary" species.

The standard (electron) coupling and degeneracy parameters defined, respectively, as

$$
\Gamma=\beta e^{2} / a, \quad D=\beta E_{F},
$$

span in strongly coupled plasmas (SCPs) two or even three orders of magnitude from about 1 to hundreds or thousands. Respectively, the Brueckner parameter $r_{s}=a / a_{B}$ might vary from about $10^{-3}$ to $10^{3}$ or even more. Here, $a_{B}, E_{F}$, and $n_{e}$ are the electron Wigner-Seitz and Bohr radii, 
the Fermi energy, and the number density of (free) electrons; besides, the temperature $T=\left(k_{B} \beta\right)^{-1}$, and $r_{s}=\frac{\Gamma}{2 D}\left(\frac{9 \pi}{4}\right)^{\frac{2}{3}}=1.842 \frac{\Gamma}{D}$.

We observe that the common property of SCPs is that the interparticle potential energy can dominate over the thermal energy while the system temperature might be comparable or exceed significantly its Fermi temperature. Despite the lack of small parameters $\left(\Gamma\right.$ or $D$ or $r_{s}$ or $\left.r_{s}^{-1}\right)$, static structural and even kinetic characteristics of strongly coupled plasmas are relatively easy to determine numerically, see, e.g., $[19,20]$. Currently there are no first-principle physical approaches capable of producing reliable results on dynamic properties of such systems within the above gaps between ideal-gas and solid-state conditions, see nevertheless [21] and references therein. Good agreement between the numerical and theoretical results in a relatively wide realm of variation of $\Gamma$ and/or $D$ is finally achieved using up to four adjustable parameters [22]. Simulation data on the dynamic localfield corrections remains unexplained theoretically [23].

The keystone of our approach is the plasma (inverse) dielectric function (IDF), $\varepsilon^{-1}(k, z=\omega+i \delta)(\delta \geq 0)$, which is the genuine response function for any wavenumber, and the even non-negative loss function (LF):

$$
L\left(k, x=\omega^{2}\right)=-\operatorname{Im} \varepsilon^{-1}(k, \omega) / \omega .
$$

We will show how on the rigorous mathematical basis complemented by simple physical considerations, the knowledge of these dynamic characteristics can be reduced to that of the static ones, precisely, the static structure factors (SSFs). The approach construction blocks are the system sum rules or the loss function frequency power moments 


$$
C_{v}(k)=\frac{1}{\pi} \int_{-\infty}^{\infty} \omega^{v} L(k, \omega) d \omega, v=0,2,4 .
$$

Notice that the odd-order moments vanish due to the symmetry of the loss function. The moments $\left\{C_{0}(k), C_{2}, C_{4}(k)\right\}$ and the characteristic frequencies

$$
\omega_{1}^{2}(k)=\frac{C_{2}(k)}{C_{0}(k)}, \omega_{2}^{2}(k)=\frac{C_{4}(k)}{C_{2}(k)} .
$$

are known independently, they are determined by the system composition, degeneracy, and thermodynamics. It should be noted, that for any pair of distribution densities, for example, $f(\omega)$ and $g(\omega)$, the next expression is correct:

$$
\left|\int_{-\infty}^{\infty} f(\omega) g(\omega) d \omega\right|^{2} \leq \int_{-\infty}^{\infty}|f(\omega)|^{2} d \omega \cdot \int_{-\infty}^{\infty}|g(\omega)|^{2} d \omega .
$$

This inequality is a particular case of the Hölder inequality in $\mathrm{L}^{2}$, or the Cauchy-Schwarz-Bunyakovsky inequality. If we choose as $|g(\omega)|=\sqrt{L(k, \omega) / \pi} \quad$ and $|f(\omega)|=\omega^{2} \sqrt{L(k, \omega) / \pi}$, we will get $C_{2}^{2}=C_{4}(k) C_{0}(k)$, or we can wtite this equation as (where $\omega_{p}$ is, of course, the system plasma frequency):

$$
b(k)=\frac{\omega_{2}(k)-\omega_{1}(k)}{\omega_{p}}>0 .
$$

This relation can help in the analysing of the static structure calculations SSF schemes, as we will show later.

The zero sum rule $C_{0}$ stems from the Kramers-Kronig relations, this result does not depend on the nature of the system:

$$
\varepsilon^{-1}(k, z)=1+\int_{-\infty}^{\infty} \frac{\omega L(k, \omega) d \omega}{\pi(z-\omega)} \Leftrightarrow C_{0}(k)=1-\varepsilon^{-1}(k, 0) .
$$


The second moment is the f-sum rule:

$$
C_{2}=\frac{1}{\pi} \int_{-\infty}^{\infty} \omega^{2} L(k, \omega) d \omega \equiv \omega_{p}^{2}
$$

The loss function fourth power moment

$$
C_{4}=\frac{1}{\pi} \int_{-\infty}^{\infty} \omega^{4} L(k, \omega) d \omega
$$

for OCPs has been studied first by Kugler and Pathak and Vashishta [24]. These results were further generalized [25] within the Kubo linear-reaction theory and using the second-quantization technique, see [26, 27]. It was established that in multicomponent electron-ion Coulomb system with the pairwise interaction energy Fourier transform the second characteristic frequency contains four contributions:

$$
\omega_{2}^{2}(k)=\omega_{p}^{2}\left[\zeta_{e e}(k)+K(k)+U(k)+H\right] .
$$

The kinetic contribution $K(q)$ consists of two parts

$$
K(k)=\frac{(k a)^{2}}{\Gamma} \frac{F_{3 / 2}(\eta)}{D^{3 / 2}}+\frac{(k a)^{4}}{12 r_{s}},
$$

and the $F_{\mu}(\eta)=\int_{0}^{\infty} \frac{t^{\mu}}{\exp (t-\eta)+1} d t$ is the order- $\mu$ Fermi integral, and $\eta$ is the dimensionless chemical potential of the electronic subsystem, which should be determined by the normalization condition $F_{1 / 2}(\eta)=\frac{2}{3} D^{3 / 2}$.

The coupling contribution $U(q)$ and correlation contribution $H$ have the forms: 


$$
\begin{gathered}
U(k)=\frac{1}{12 \pi} \int_{0}^{\infty} p^{2}\left(S_{e e}(p)-1\right)\left(Z_{e e}(p, k)-\frac{8 \zeta_{e e}(p)}{3}\right) d p, \\
H=\frac{2 \sqrt{Z}}{9 \pi} \int_{0}^{\infty} p^{2} S_{e i}(p) \zeta_{e i}(p) d p,
\end{gathered}
$$

where $\mathrm{Z}$ is a special function, which is dependent from the system, via the $\zeta_{a b}$ - factor:

$$
Z_{e e}(p, k)=\int_{|p-k|}^{p+k} \zeta_{e e}(s)\left(p^{2}-k^{2}-s^{2}\right)^{2} \frac{d s}{p k^{3} s} .
$$

Notice that the contribution $\mathrm{H}$ is $k$-independent, it is determined by the partial static structure factor corresponding to the coupling of the electron subsystem with the lightest ion species. It is obvious that in one-component plasmas, either classical or not, the electron-ion correction $\mathrm{H}=0$.

Notice also that in the hydrodynamic limiting case

$$
\omega_{2}^{2}(k \rightarrow 0) \simeq \omega_{p}^{2}(1+H),
$$

while at short distances we recover the single-particle behavior:

$$
\omega_{2}^{2}(k \rightarrow \infty) \simeq \frac{\omega_{p}^{2}(k a)^{4}}{12 r_{s}} .
$$

The frequencies $\omega_{1}(k)$ and $\omega_{2}(k)$ are the construction blocks of our approach. In order to find the dynamic characteristics of Coulomb systems and to relate them to the static ones, we use the solutions of the truncated Hamburger moment problem corresponding to five convergent frequency moments $\left\{C_{0}(k), 0, C_{2}, O, C_{4}(k)\right\}$.

Note also that the r.h.s. of the Nevanlinna formula can be presented as a truncated continuous fraction: 


$$
\int_{-\infty}^{\infty} \frac{L(k, \omega) d \omega}{\pi(\omega-z)}=C_{0}\left(z-\omega_{1}^{2}\left(z-\frac{\omega_{2}^{2}-\omega_{1}^{2}}{z+Q}\right)^{-1}\right)^{-1}
$$

or we can write as:

$$
\int_{-\infty}^{\infty} \frac{L(k, \omega) d \omega}{\pi C_{0}(\omega-z)}=\frac{\omega_{2}^{2}-\omega_{1}^{2}-z(z+R)}{z\left(z^{2}-\omega_{2}^{2}\right)+R\left(z^{2}-\omega_{1}^{2}\right)},
$$

which establishes a one-to-one correspondence (a bijection) between the Nevanlinna parameter functions $R(k, z)$ and the non-canonical solutions of the moment problem for the loss function. For the case of $\mathrm{z} \rightarrow \infty$ we have

$$
\int_{-\infty}^{\infty} \frac{L(k, \omega) d \omega}{\pi C_{0}(\omega-z)}=-\frac{1}{\pi C_{0}(k) z} \int_{-\infty}^{\infty} \frac{L(k, \omega) d \omega}{1-\omega / z} \underset{z \rightarrow \infty}{\simeq}-\frac{1}{2}-\frac{\omega_{1}^{2}}{z^{3}}-\frac{\omega_{1}^{2} \omega_{2}^{2}}{z^{5}}+\ldots
$$

On the other hand, we can write an equation

$$
\frac{\omega_{2}^{2}-\omega_{1}^{2}-z(z+R)}{z\left(z^{2}-\omega_{2}^{2}\right)+R\left(z^{2}-\omega_{1}^{2}\right)} \underset{z \rightarrow \infty}{\simeq}-\frac{1}{2}-\frac{\omega_{1}^{2}}{z^{3}}-\frac{\omega_{1}^{2} \omega_{2}^{2}}{z^{5}}\left[1-\frac{R}{z}\left(1-\frac{\omega_{1}^{2}}{\omega_{2}^{2}}\right)\right]-\ldots
$$

So, we have that

$$
\frac{L(k, \omega)}{\pi C_{0}(k)}=\frac{\omega_{1}^{2}\left(\omega_{2}^{2}-\omega_{1}^{2}\right) \operatorname{Im} R}{\left|\omega\left(\omega^{2}-\omega_{2}^{2}\right)+R\left(\omega^{2}-\omega_{1}^{2}\right)\right|^{2}} .
$$

The dielectric function itself has a form:

$$
\varepsilon(k, z)=1-\frac{\omega_{p}^{2}(z+R)}{z\left(z^{2}-\omega_{2}^{2}+\omega_{p}^{2}\right)+R\left(z^{2}-\omega_{1}^{2}+\omega_{p}^{2}\right)}, \operatorname{Im} z>0 .
$$

The simplest is to model the NPF $R$ by its static value, like it was done in [28]:

$$
R(k, z)=R(k, 0)=i h(k), h(k)>0 .
$$

The function $R(k, z)$ should additionally satisfy the limiting condition: 


$$
\frac{R(k, z)}{z} \underset{z \uparrow \infty}{\rightarrow} 0, \operatorname{Im} z>0
$$

Using the static approximation of NPF, we have

$$
\left.\frac{L(k, \omega)}{\pi C_{0}(k)}\right|_{Q=i h}=\frac{\omega_{1}^{2}\left(\omega_{2}^{2}-\omega_{1}^{2}\right) h}{\omega^{2}\left(\omega^{2}-\omega_{2}^{2}\right)^{2}+h^{2}\left(\omega^{2}-\omega_{1}^{2}\right)^{2}} .
$$

The main problem is to derive the expression for the NPF. In [28] the NPF was approximated by the form $R=i \tau^{-1}$, where $\tau$ is the relaxation time. This time was selected to reproduce an exact static value of the dynamic structure factor, in other words, there was a connection with simulation data. In the present work we derive another representation of NPF, without any connection with simulation data.

Consider the Fourier transform of the loss function, $\Lambda(k, t)$, whose behavior at long times, by virtue of the Tauber or Abel theorems, will be similar to that of the DSF Fourier transform. The function $\Lambda(k, t)$ is bounded, decreasing exponentially and becomes essentially zero for long times. Taking into account the physical time scales of the problem, for time $t$ larger than the longest relaxation time of the system collective modes and in compliance with Bogolyubov's principle of weakening of correlations, $\Lambda(k, t)$ has a finite (zero) limit as $t \rightarrow \infty$. Then $L(k, \omega)$ like the DSF, also has a finite zero-frequency limiting value. Hence, for very low frequencies, due to the same theorems, the values of the loss function need to be weakly dependent on $\omega$. Besides, since the loss function is an odd function of frequency, all of its odd-order frequency derivatives vanish at $\omega=0$. Therefore, the second derivative of $L(k, \omega)$ with respect to $\omega$ (or the first derivative with respect to $\omega^{2}$ ) should be negligible in the limit of very low frequencies:

$$
\left.\frac{d^{2} L(k, \omega)}{d \omega^{2}}\right|_{\omega=0}=\left.\frac{d L(k, x)}{d x}\right|_{x=\omega^{2}=0}=0, \operatorname{Im} z>0 .
$$


The above qualitative physical justification of the semi-empirical observation: the loss function as an even function of frequency or as a function of $x=\omega^{2}$ possesses a relatively broad extremum at $\omega=0$.

We can rewrite (3.22) as:

$$
\left.\frac{L(k, \omega)}{\pi C_{0}(k)}\right|_{Q=i h}=: \omega_{1}^{2}\left(\omega_{2}^{2}-\omega_{1}^{2}\right) h \Phi\left(\omega^{2} ; h\right) .
$$

Due to the symmetry of the loss function, the auxiliary function $\Phi\left(\omega^{2} ; \mathrm{h}\right)$ depends only on frequency squared and the function $\Phi\left(x=\omega^{2} ; h\right)$ exhibits an extremum at $\mathrm{x}=0$ if

$$
\left.\frac{d \Phi(x ; h)}{d x}\right|_{x=0}=\frac{2 h^{2} \omega_{1}^{2}-\omega_{2}^{2}}{h^{4} \omega_{1}^{8}}=0 .
$$

If we solve this equation, we will get a new value for the $h$ :

$$
h(k)=h_{0}(k)=\frac{\omega_{2}^{2}(k)}{\omega_{1}(k) \sqrt{2}},
$$

which is the main result of the present work. This value transform the classical method of moments to the self-consistent moments method. In addition, the Nevanlinna parameter $h$ can be determined by the procedure of maximization of the Shannon entropy:

$$
S(h)=\int_{0}^{\infty} L(\omega, h) \ln L(k a, \omega, h) d \omega .
$$

We have found, that $\mathrm{h}_{0}$ is quite close to the true maximization value of the Shannon entropy parameter. In other words, the substitution of the free parameter $h$ by its physically motivated value $h_{0}$ does not contradict the Shannon entropy maximization principle, see Fig. 3.1. In this graph we used dimensionless wavenumber $q=k a$. 


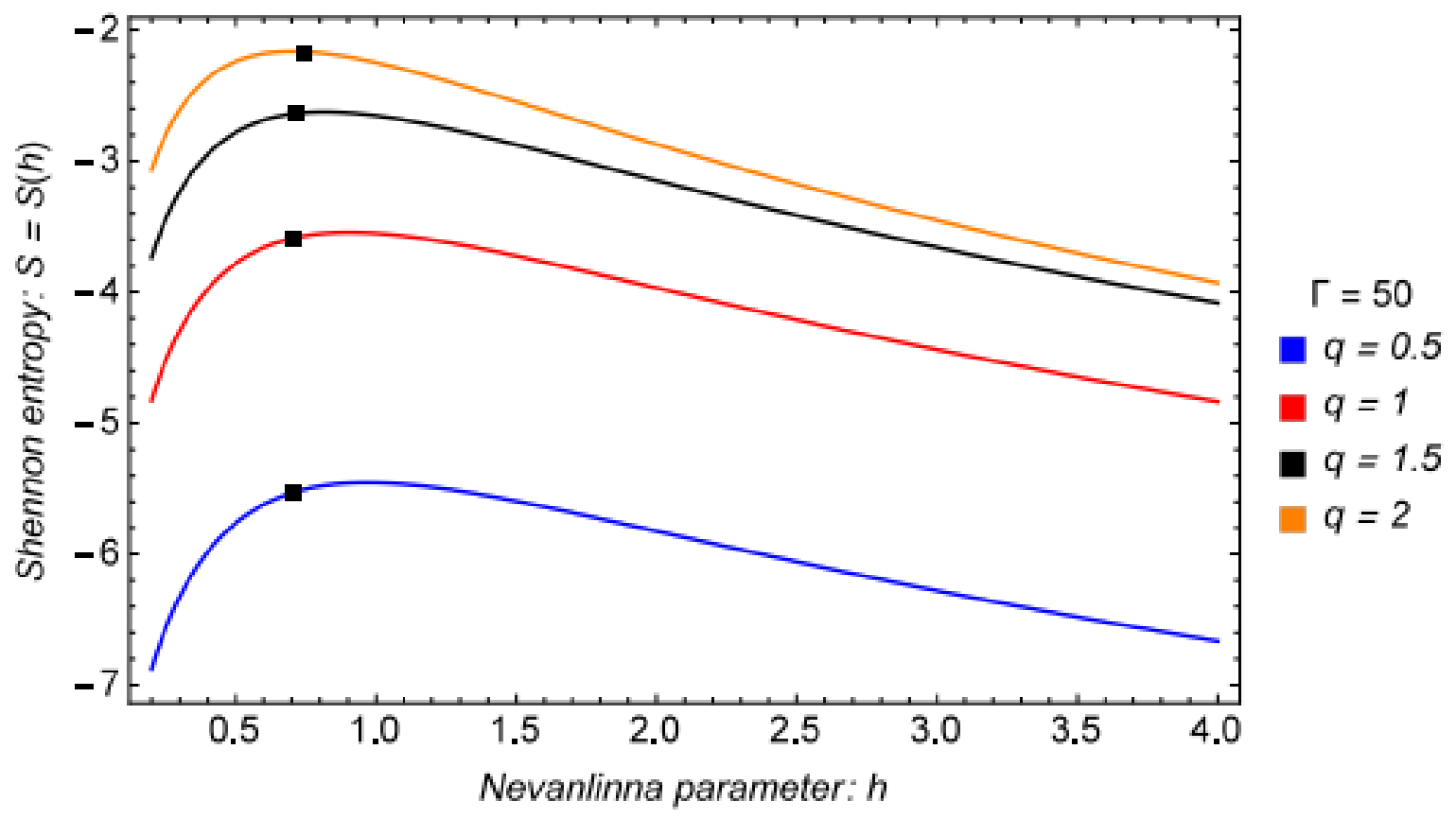

Figure 3.1. The COCP Shannon entropy as a function of the Nevanlinna parameter $h(q)$ for different wavenumber values. Squares stand for $S\left(h_{0}(q)\right)$ value. 
The sign of the second derivative at $\mathrm{x}=0$ for $\mathrm{h}=\mathrm{h}_{0}$ :

$$
\left.\frac{d^{2} \Phi\left(x ; h_{0}\right)}{d x^{2}}\right|_{x=0}=\frac{4\left(\omega_{1}^{2}-\omega_{2}^{2}\right)}{\omega_{1}^{6} \omega_{2}^{6}} .
$$

In other words, the sign of parameter

$$
\theta(k)=\frac{2 \omega_{1}(k)-\omega_{2}(k)}{\omega_{p}} \in\left(-\frac{\omega_{2}(k)}{\omega_{p}}, \frac{\omega_{2}(k)}{\omega_{p}}\right),
$$

determines the nature of the extremum at $\omega=0$ : a positive $\theta(k)$ corresponds to a minimum, and a negative value to a maximum. But beside the minimum that occurs for the positive $\theta(k)$, there is a shifted maximum and that in nonor slightly degenerate systems its psition on the wave axes is determined by following relation

$$
\omega_{m}(k)= \pm \frac{\omega_{2}(k)}{\omega_{1}(k) \sqrt{3}} \sqrt{\theta(k) \omega_{p}\left(2 \omega_{1}(k)+\omega_{2}(k)\right)} .
$$

The important result (3.26) leads to the following simple form for the loss function:

$$
\left.\frac{L(k, \omega)}{\pi C_{0}(k)}\right|_{Q=i h}=\frac{\omega_{1}^{2}\left(\omega_{2}^{2}-\omega_{1}^{2}\right) h_{0}}{\omega^{2}\left(\omega^{2}-\omega_{2}^{2}\right)^{2}+h_{0}^{2}\left(\omega^{2}-\omega_{1}^{2}\right)^{2}} .
$$

Thus, we observe that the Nevanlinna theorem permits to reduce the search for the loss function to the study of the NPF or only of the static parameter function $h(k)$.

In the next chapter we will theoretically calculate the OCP dynamic structure factors, find the dispersion relation and describe the moleculardynamics (MD) data for the local field corrections. 


\section{SOLUTION OF PHYSICAL PROBLEMS BY THE SELF- CONSISTENT METHOD OF MOMENTS}

\subsection{Dynamic structure factors}

Here we demonstrate that the method of moments theoretical approach is able to predict the form and structure of the DSF of the classical OCP, based on static data only, i.e., the static structure factor (SSF). The SSF can be obtained theoretically within the hypernetted chain (HNC) approximation and its modifications including the bridge function. The present approach provides a purely theoretical access to the full DSF and a full quantitative description of the collective modes, including their decay and other characteristics, without the necessity to use simulation data as input.

The dynamic structure factor "charge-charge" (DSF), which is the central quantity of collective and dynamic effects, is determined by the loss function via the classical fluctuation-dissipation theorem (FDT):

$$
S(k, \omega)=\frac{L(k, \omega)}{\pi \beta \phi(k)},
$$

so that the moments of DSF are proportional, for a given value of the wave number, to the corresponding moments of the loss function (2.3) in the following way:

$$
S_{v}(k)=\frac{k^{2}}{k_{D}^{2}} C_{v}(k)
$$

The zero-order moment is, obviously, the SSF, $S_{0}=S(k)$, while the second moment is the $f$-sum rule 


$$
S_{2}(k)=\omega_{p}^{2}\left(\frac{k^{2}}{k_{D}^{2}}\right)
$$

The fourth moment of DSF equals to

$$
S_{4}(k)=\omega_{p}^{4}\left(\frac{k^{2}}{k_{D}^{2}}\right)\left(\xi(k)+3 \frac{k^{2}}{k_{D}^{2}}+U(k)\right) .
$$

The characteristics frequencies are, therefore

$$
\begin{gathered}
\frac{\omega_{1}^{2}(q)}{\omega_{p}^{2}}=\frac{q^{2} \omega_{p}^{2}}{3 \Gamma S(q)}, \\
\frac{\omega_{2}^{2}(q)}{\omega_{p}^{2}}=\zeta(q)+\frac{q^{2}}{\Gamma}+\frac{1}{12 \pi} \int_{0}^{\infty} p^{2}(S(p)-1) f(p) d p,
\end{gathered}
$$

where $q=k a$, and $f(p)$ function equals to

$$
f(p)=\left(\int_{|p-q|}^{p+q} \frac{\zeta(s)\left(p^{2}-q^{2}-s^{2}\right)^{2} d s}{p q^{3} s}-\frac{8 \zeta(p)}{3}\right) .
$$

Therefore, the DSF can be wtitten in the

$$
\frac{\pi S(q, \omega)}{n S(q)}=\frac{\omega_{1}^{2}\left(\omega_{2}^{2}-\omega_{1}^{2}\right) h_{0}}{\omega^{2}\left(\omega^{2}-\omega_{2}^{2}\right)^{2}+h_{0}^{2}\left(\omega^{2}-\omega_{1}^{2}\right)^{2}} .
$$

It implies that we can calculate the DSF and the collective mode characteristics entirely in terms of the static structure factor (SSF), this fact defines the self-consistency of the method of moments. The static characteristics are to be calculated independently, e.g., in the hypernetted chain HNC approximation, or fitting of HNC-results [29-31]. Later we will analyze several shemes for SSF calculation. 
In Figs. 4.1 - 4.10 we display results for the dynamic structure factor (DSF) of the COCP and YOCP, compared to the MD data [32,33]. In graphs we used dimensionless wavenumber $q=k a$. These and other results displayed here were obtained using the SSF calculated within the HNC approximation, or fitting models. The molecular dynamics (MD) code in [32] simulates the motion of $\mathrm{N}=10000$ pointlike particles within a cubic cell. For the COCP case they used the particle-particle particle-mesh method to account for the long range of the Coulomb potential, while for the YOCP the fast decay of the interaction forces makes it possible to introduce a cutoff distance, beyond which the interaction of particle pairs can be neglected. The integration of the equations of motion is performed with the velocity-Verlet scheme. At the initialization of the simulations the positions of the particles are set randomly, while their initial velocity vectors are sampled from a Maxwellian distribution corresponding to a specified system temperature. During the first phase of the simulations the particle velocities are rescaled in each time step, in order to reach the desired temperature.

Our results displayed here were obtained using the SSF calculated within the HNC approximation. We have studied the dependence of the quality of our dynamic results on the method of precalculation of the SSF. Several different static approaches were analyzed, we will discuss them in the third chapter. 


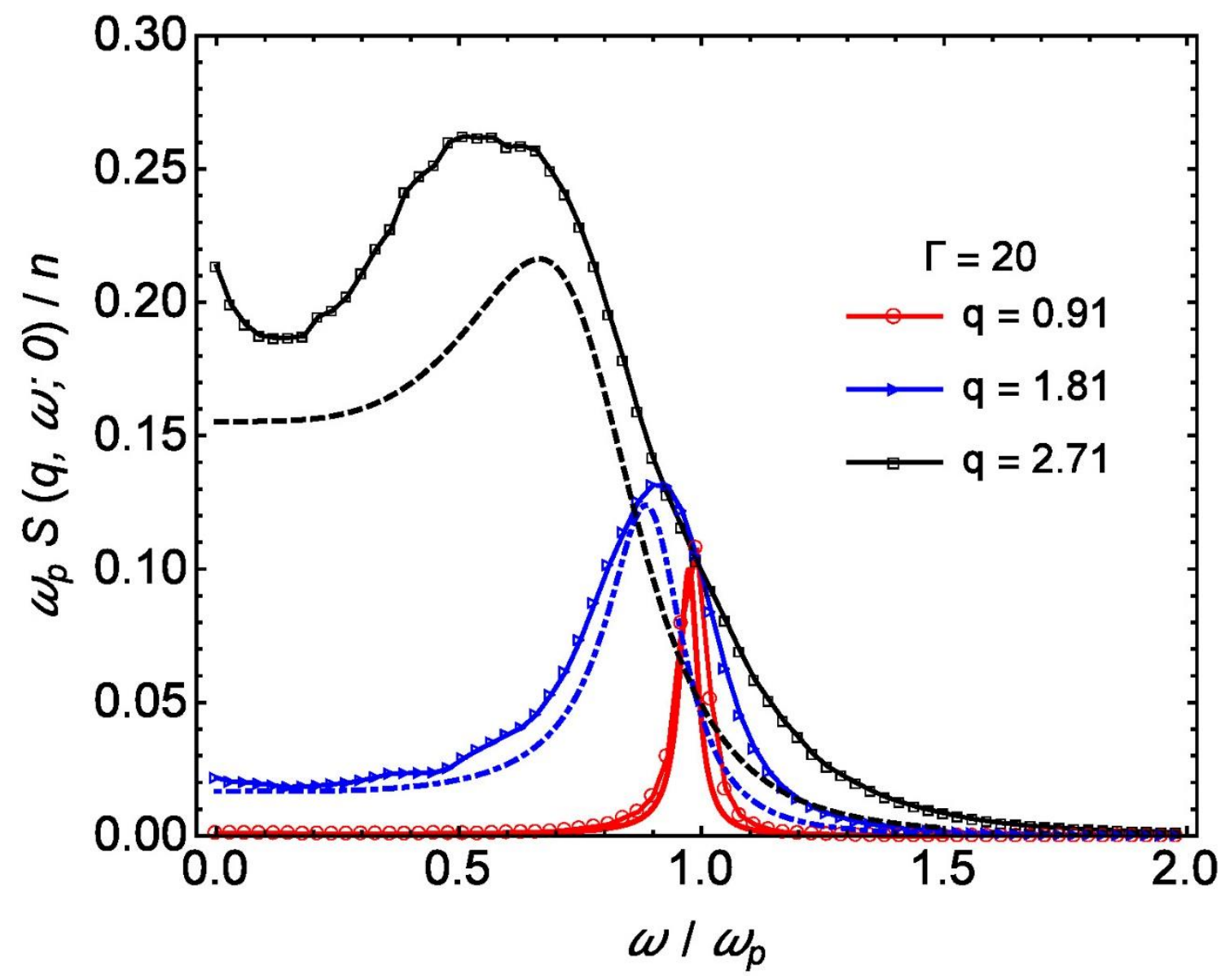

Figure 4.1. The comparison of COCP DSFs, calculated as eq. (4.7) (lines) and obtained from MD simulations [32] (lines with symbols). 


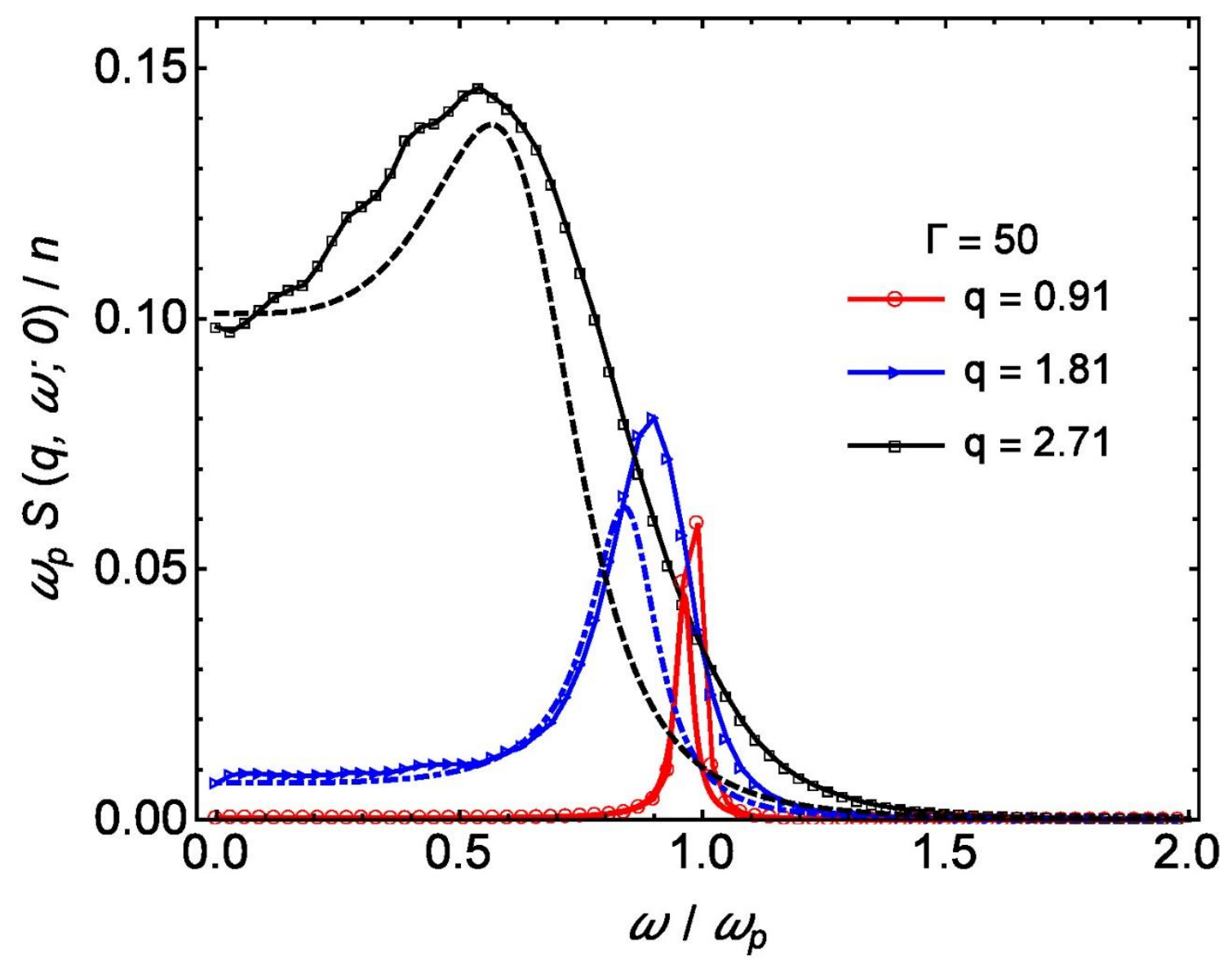

Figure 4.2. The comparison of COCP DSFs, calculated as eq. (4.7) (lines) and obtained from MD simulations [32] (lines with symbols). 


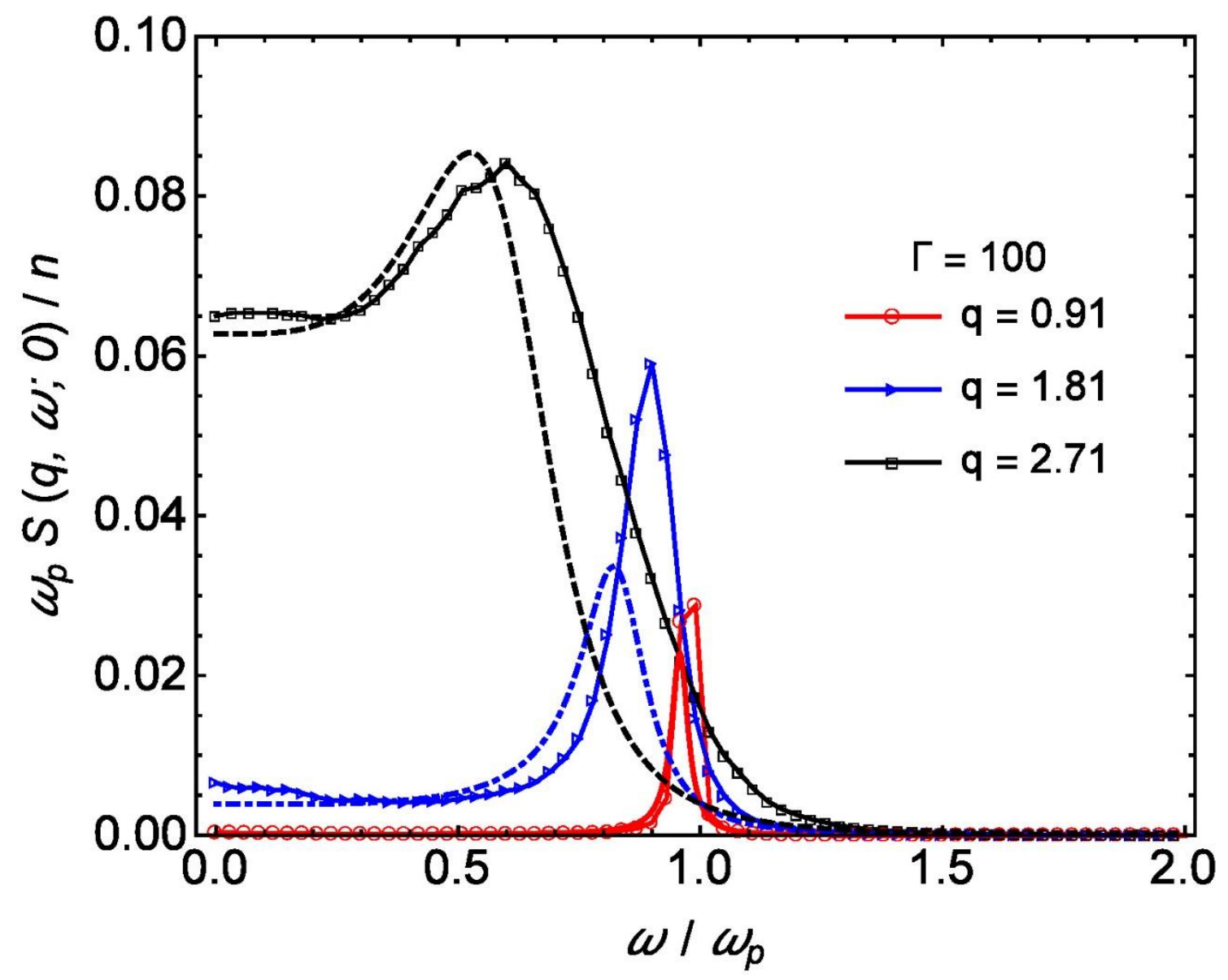

Figure 4.3. The comparison of COCP DSFs, calculated as eq. (4.7) (lines) and obtained from MD simulations [32] (lines with symbols). 


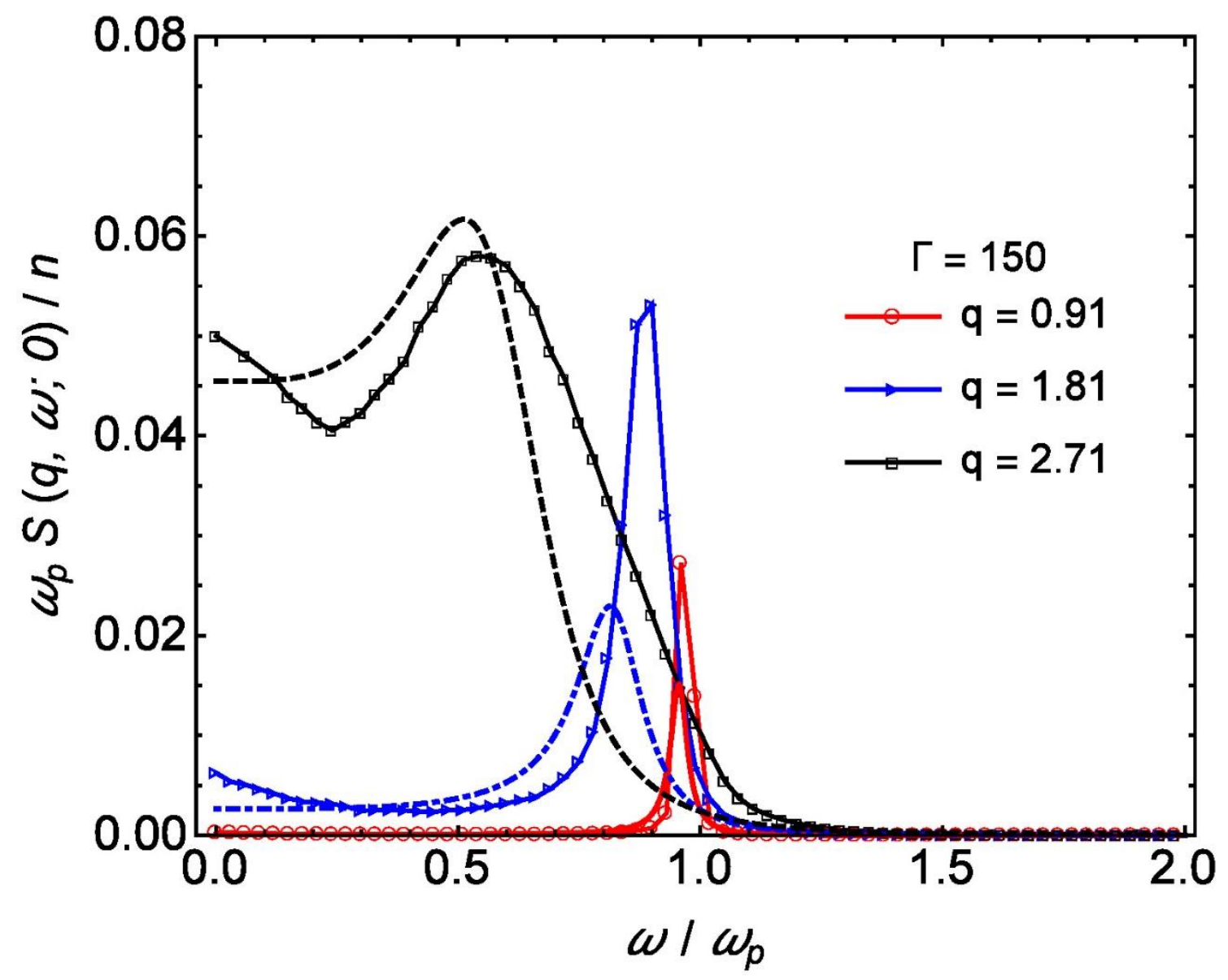

Figure 4.4. The comparison of COCP DSFs, calculated as eq. (4.7) (lines) and obtained from MD simulations [32] (lines with symbols). 

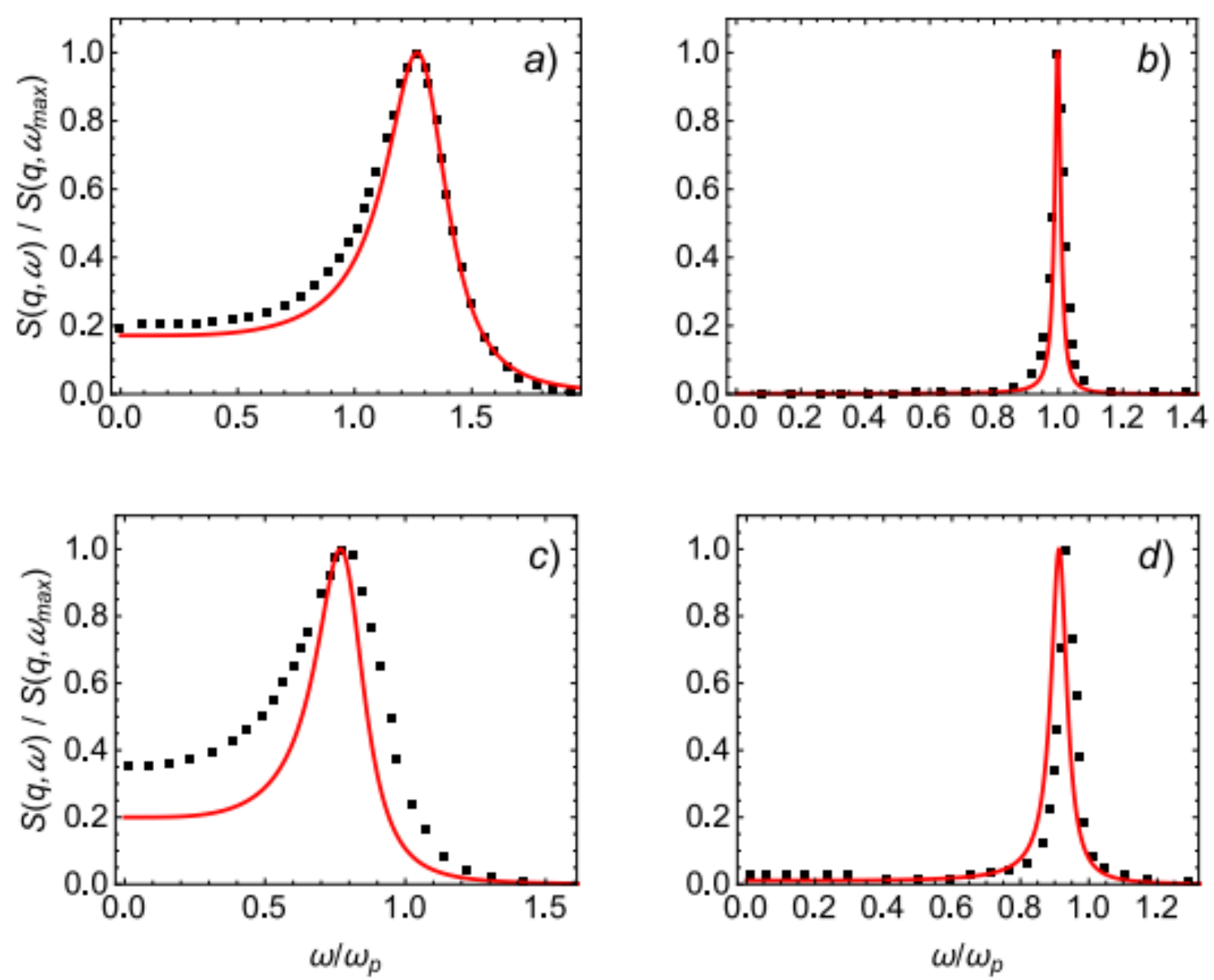

Figure 4.5. The comparison of COCP DSFs, calculated as eq. (4.7) (solid lines) and obtained from MD simulations [33] (squares),
at a) $\Gamma=1, \mathrm{q}=1.02$;
b) $\Gamma=10, q=0.64$;
c) $\Gamma=50, q=2.32$;
d) $\Gamma=120, q=1.39$. 


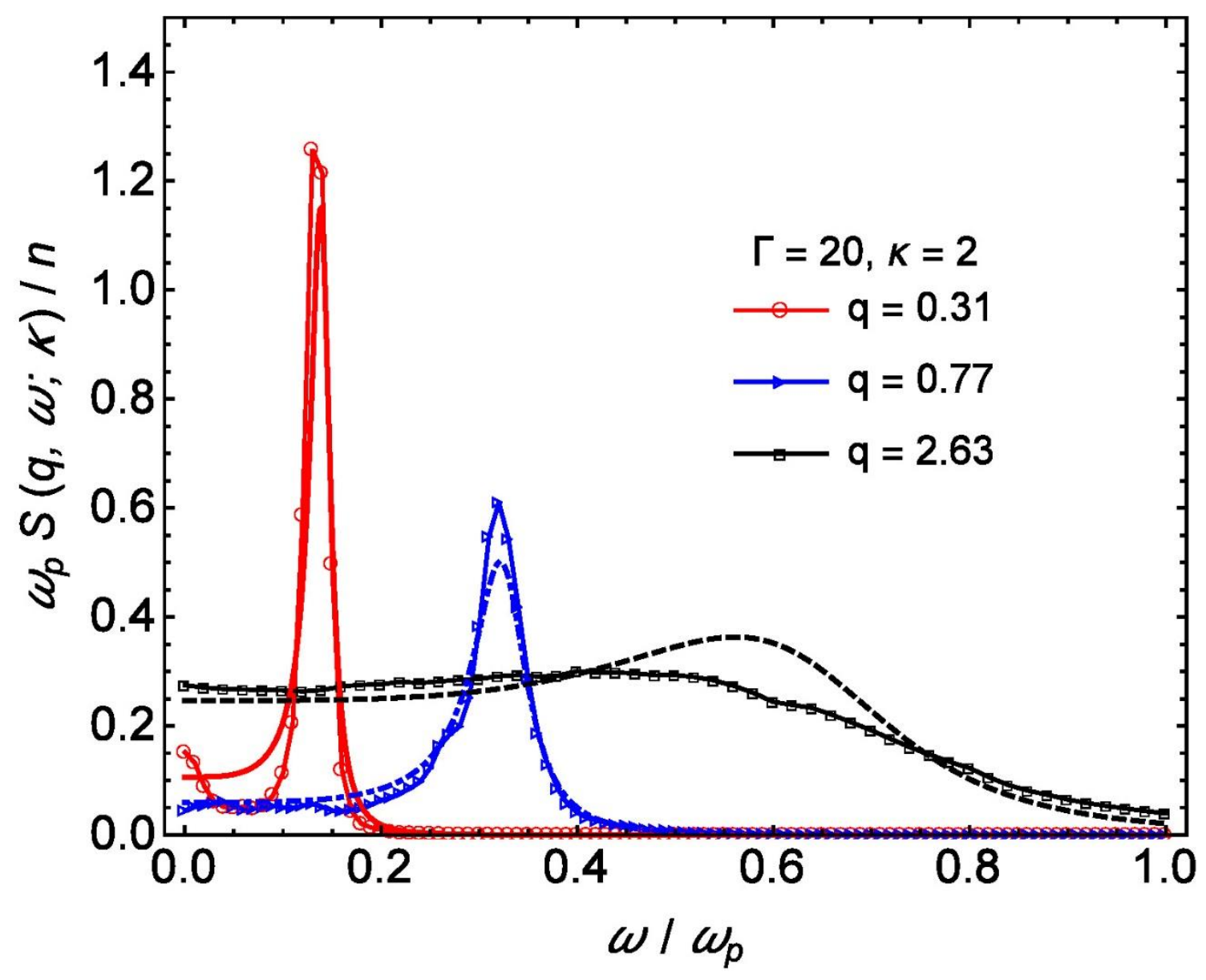

Figure 4.6. The comparison of YOCP DSFs, calculated as eq. (4.7) (lines) and obtained from MD simulations [32] (lines with symbols). 


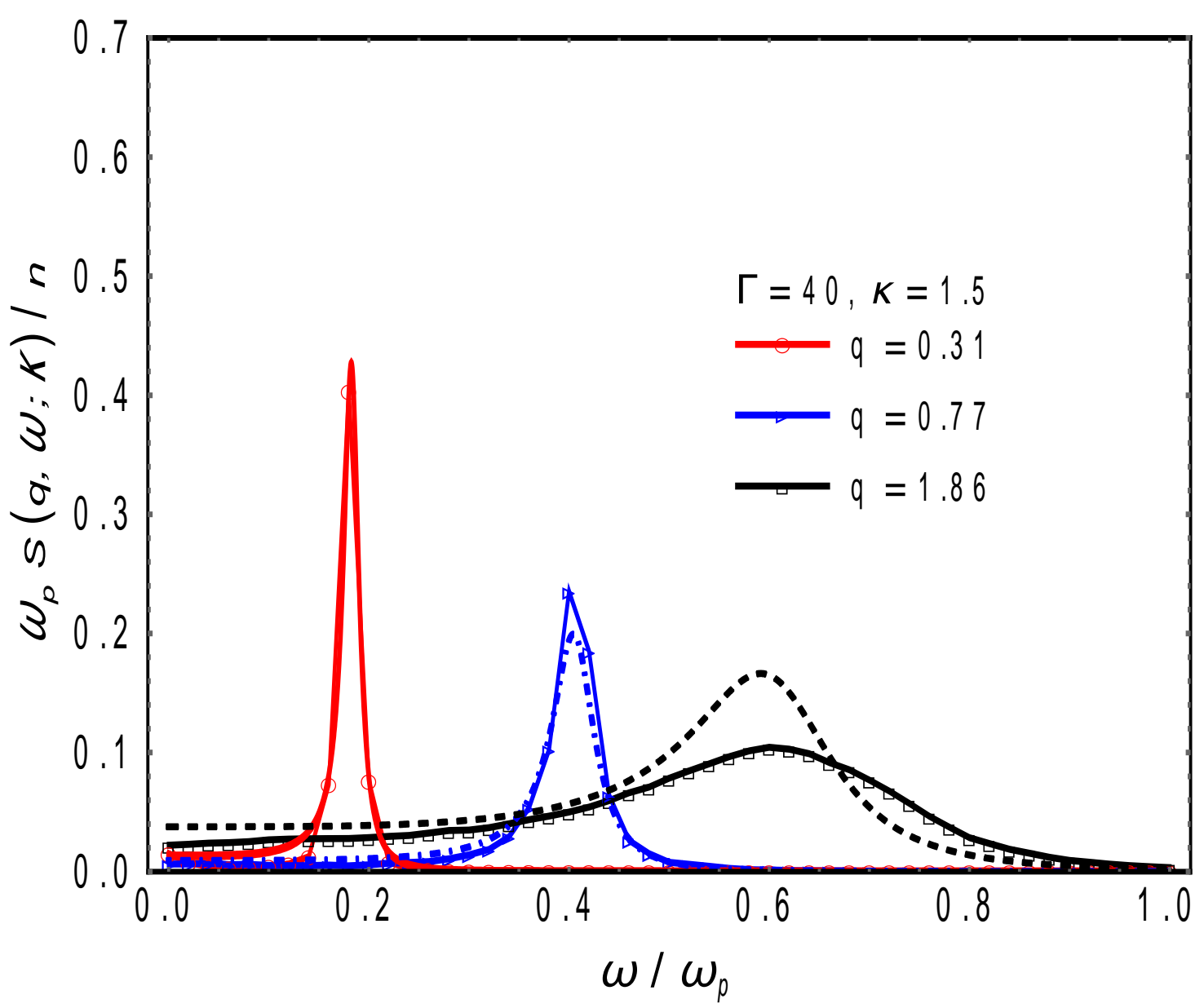

Figure 4.7. The comparison of YOCP DSFs, calculated as eq. (4.7) (lines) and obtained from MD simulations [32] (lines with symbols). 


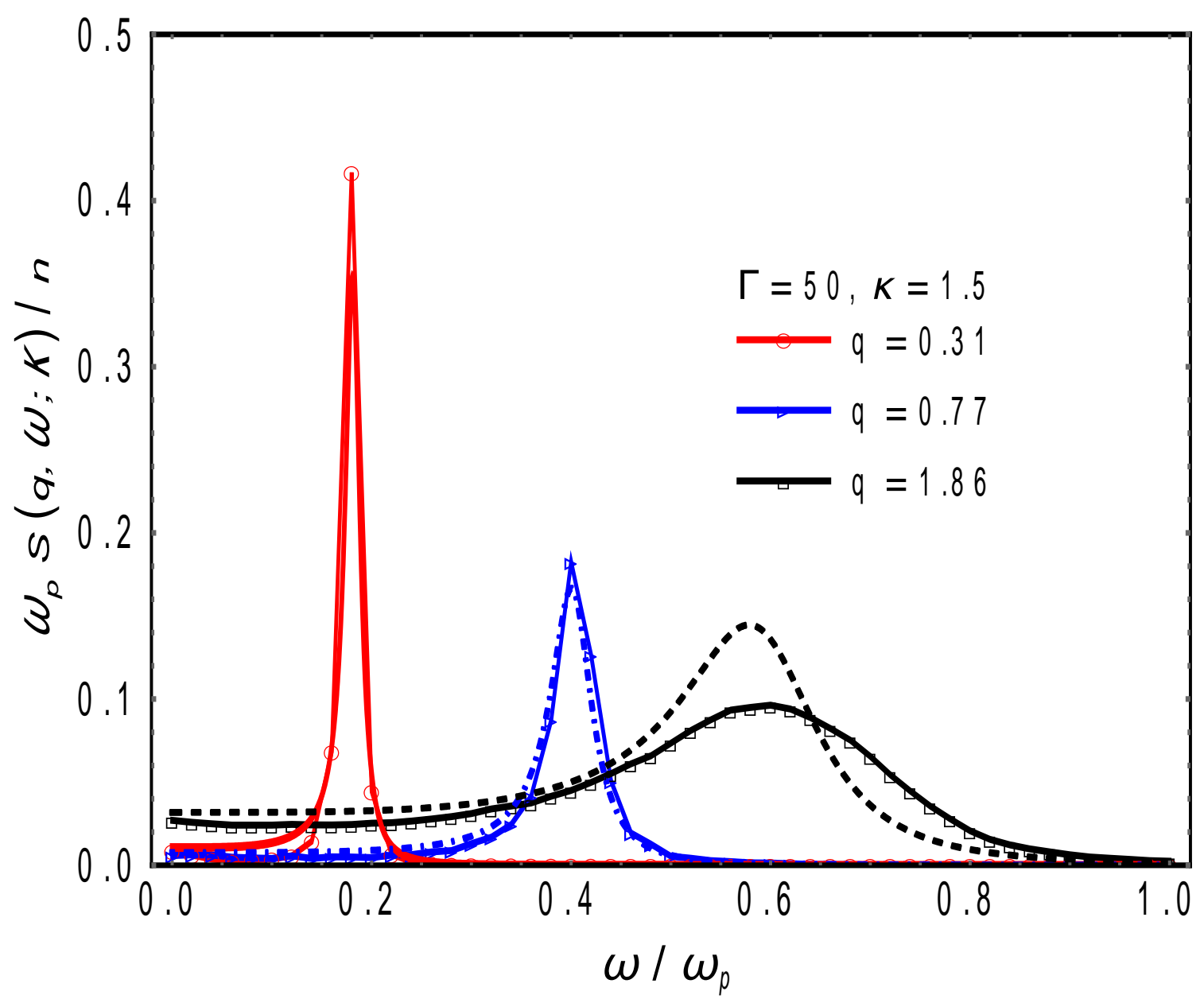

Figure 4.8. The comparison of YOCP DSFs, calculated as eq. (4.7) (lines) and obtained from MD simulations [32] (lines with symbols). 


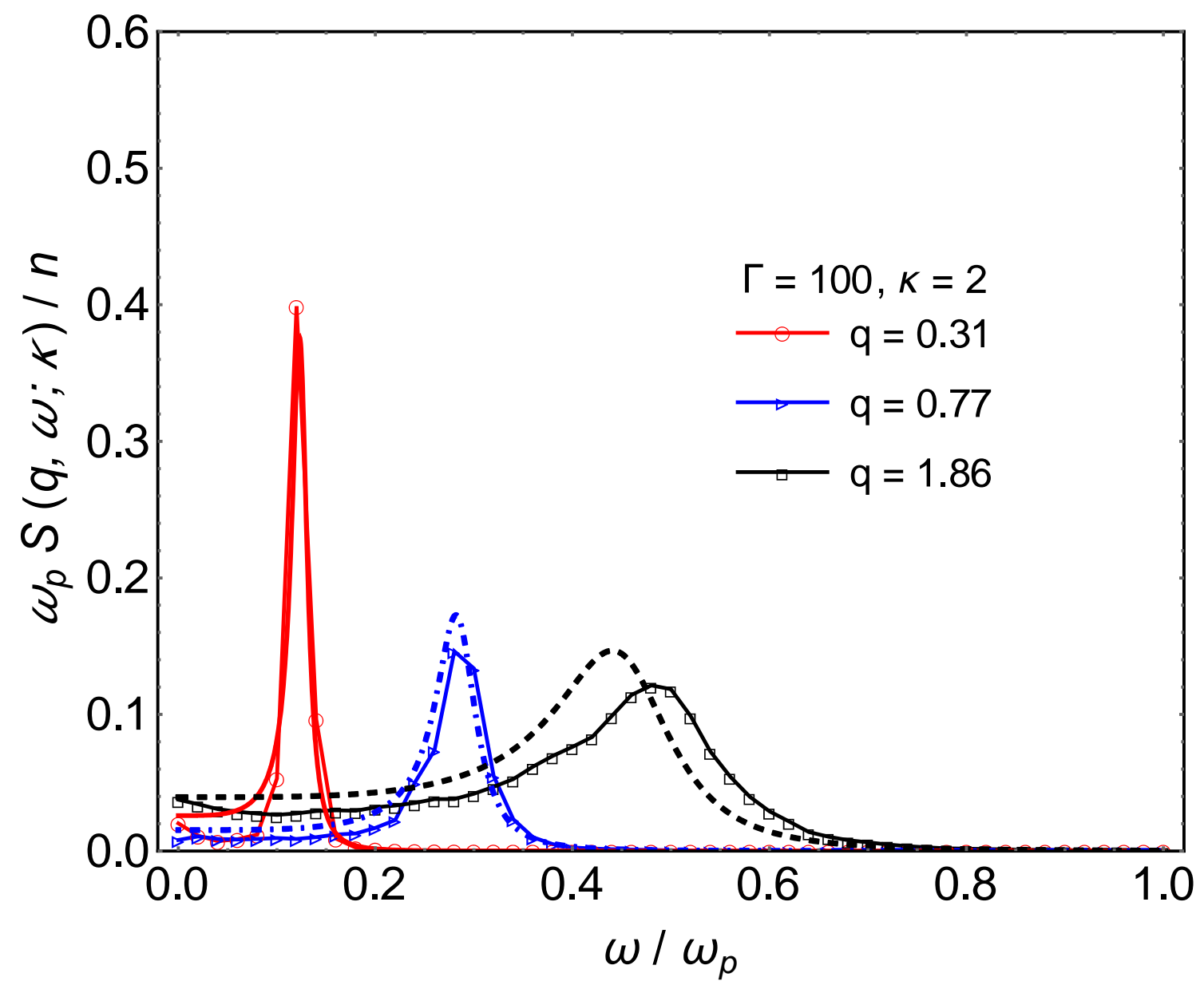

Figure 4.9. The comparison of YOCP DSFs, calculated as eq. (4.7) (lines) and obtained from MD simulations [32] (lines with symbols). 

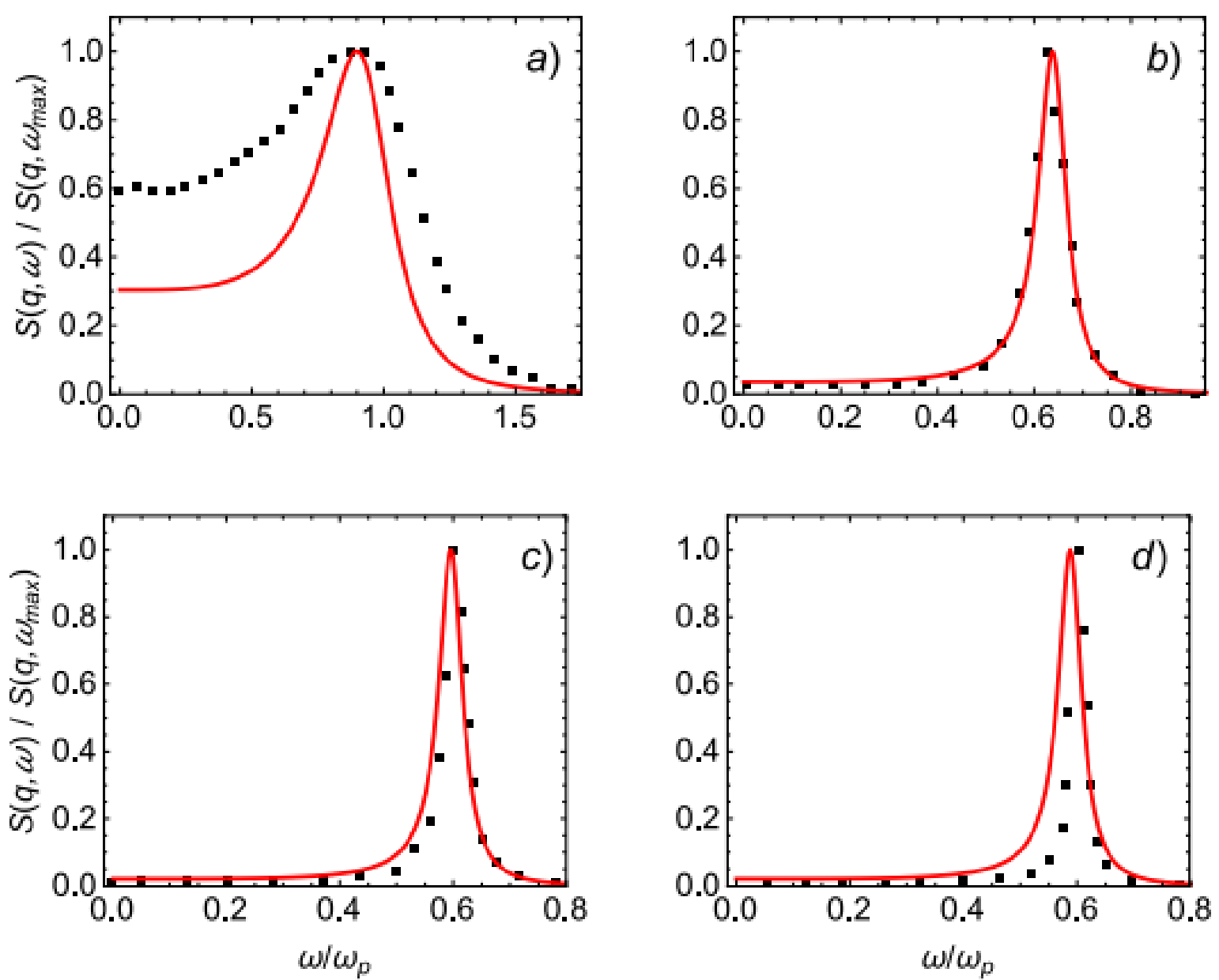

Figure 4.10. The comparison of YOCP DSFs, calculated as eq. (4.7) (solid lines) and obtained from MD simulations [33] (squares), at $\kappa=1$ and $q=0.85$ and a) $\Gamma=1$, b) $\Gamma=10$, c) $\Gamma=50$, d) $\Gamma=120$. 


\subsection{Dispersion equation}

Here we will show, how the self-consistent method of moments can be used for the investigation of collective mode propogation. In order to do this, one has to solve the dispersion equation:

$$
\omega^{2}\left(\omega^{2}-\omega_{2}^{2}(q)\right)+R(q, \omega)\left(\omega^{2}-\omega_{1}^{2}(q)\right)=0,
$$

or, if we use the approximation (2.21) with (2.26), we will get:

$$
\omega^{2}\left(\omega^{2}-\omega_{2}^{2}(q)\right)+h_{0}(q)\left(\omega^{2}-\omega_{1}^{2}(q)\right)=0 .
$$

The complex zeros of the dispersion equation (4.9) can be derived in the form:

$$
\begin{gathered}
\omega_{s h}(q)=\omega_{q}(q)-\gamma_{s h}(q)=-w X-w^{2} Y-i h_{0} / 3, \\
\omega_{u s}(q)=-\gamma_{u s}(q)=-X-Y-i h_{0} / 3 .
\end{gathered}
$$

These roots provide direct information on the system diffusion (unshifted) and acoustic-roton (shifted) modes. Here, we use $w=\exp (2 \pi i / 3)$ and

$$
\begin{gathered}
X=\sqrt[3]{\frac{h_{0} W^{2}}{2 i}+Z^{3}}, Y=\sqrt[3]{\frac{h_{0} W^{2}}{2 i}-Z^{3}}, \\
Z^{3}=\sqrt{-\left(\omega_{2}^{2} / 3-h_{0}^{2} / 9\right)^{3}-\left(h_{0} W^{2} / 2\right)^{2}}, \\
\mathrm{~W}^{2}=-\omega_{2}^{2} / 3+\omega_{1}^{2}+2 h_{0}^{2} / 27 .
\end{gathered}
$$

In Figs. $4.11-4.13$ we display results for the dispersion relation of the COCP and YOCP, compared to the MD data (Fig. 4.14). These and other results displayed here were obtained using the SSF calculated within the HNC approximation, or fitting models. Notice that no limitation on the values of $\Gamma$ (except that the system must be fluid) exists in this approach. Also, the modes can be weakly/strongly decaying or merge, they might be even non-propagating. 


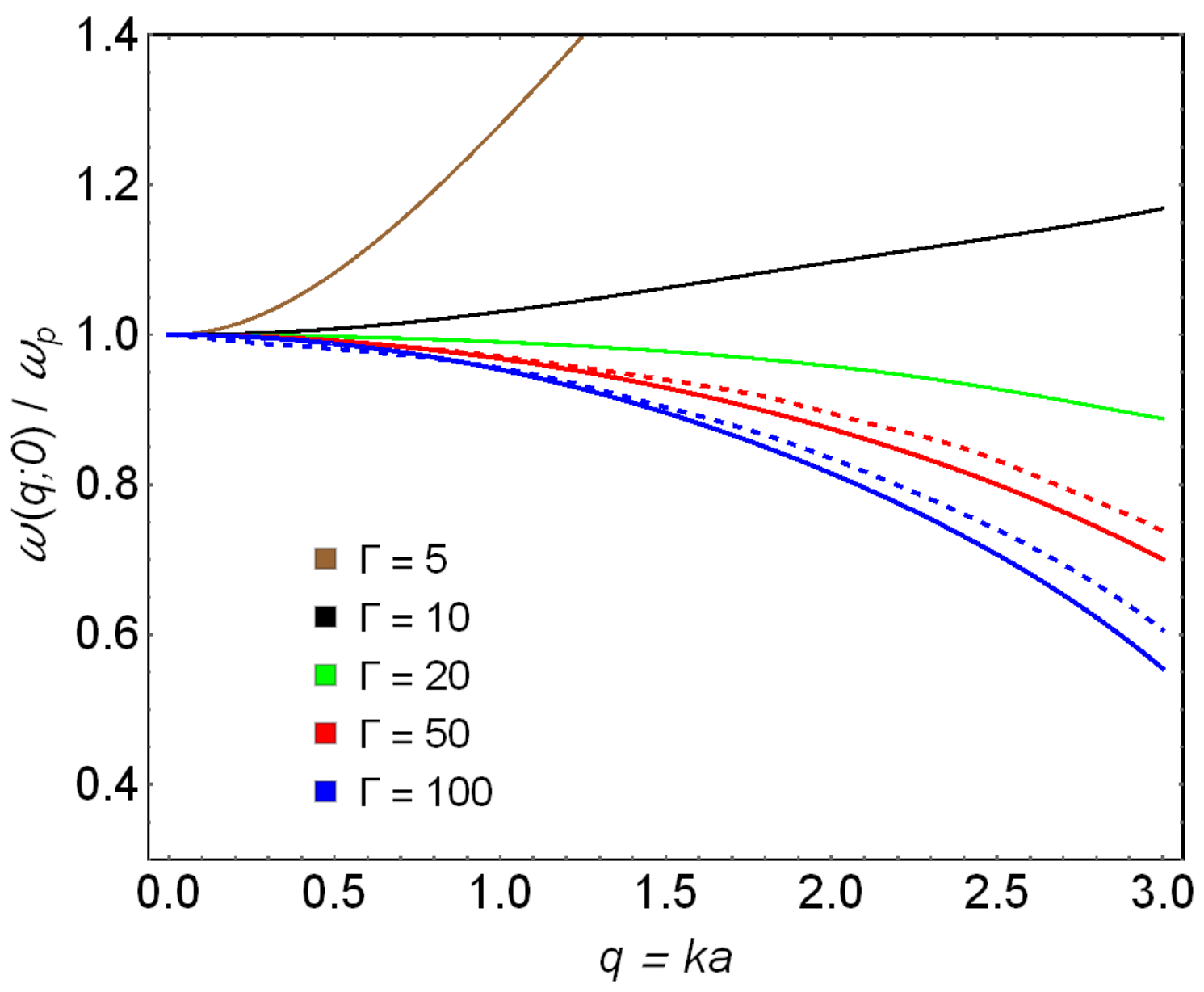

Figure 4.11. Dispersion relation for the COCP mode. Solid lines represent the exact solution of (4.9), dashed lines represent the exact solution of (4.9), but with moments, calculated from MD DSFs of [32]. 


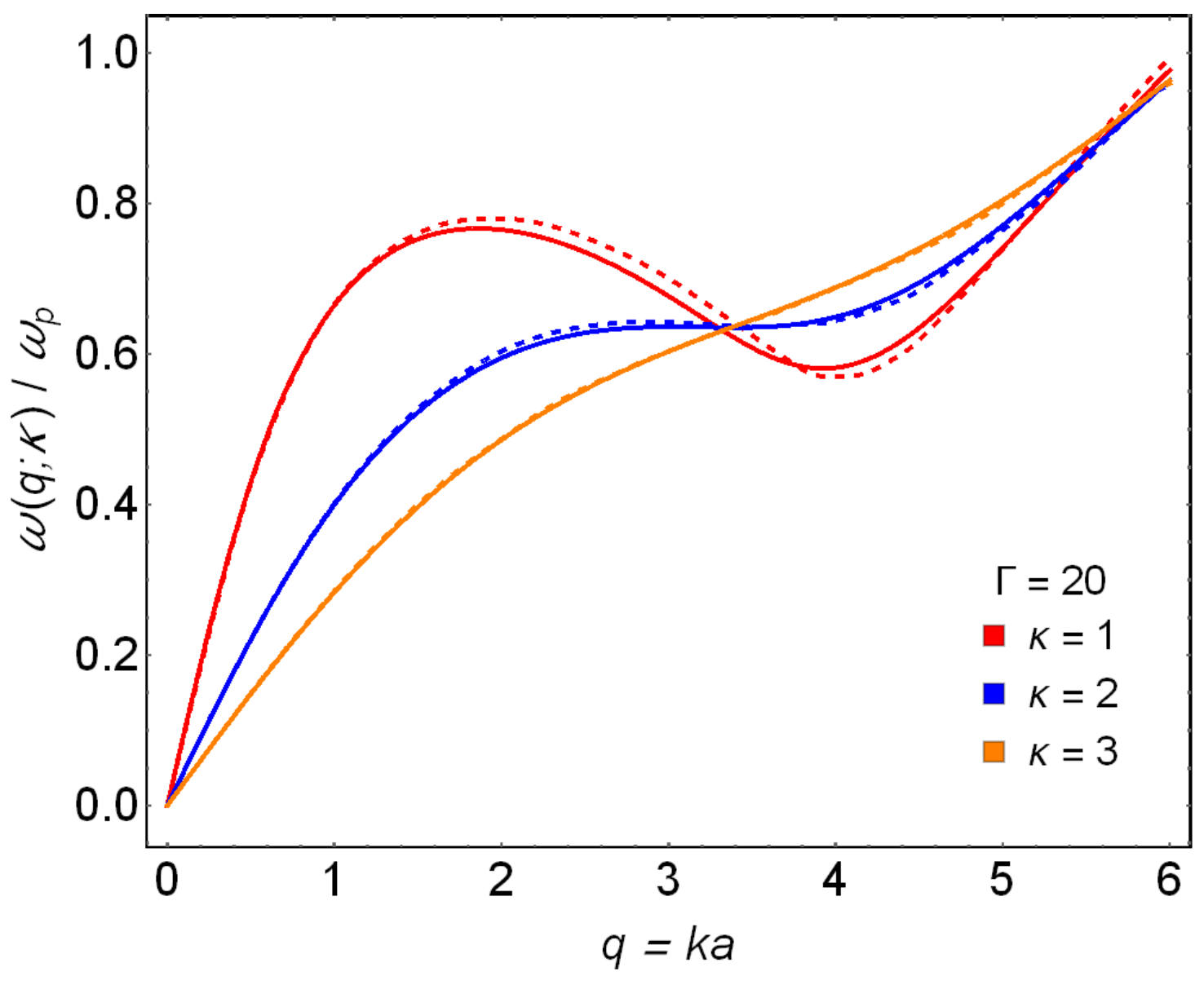

Figure 4.12. Dispersion relation for the YOCP mode. Solid lines represent the exact solution of (4.9), dashed lines represent the exact solution of (4.9), but with moments, calculated from MD DSFs of [32]. 


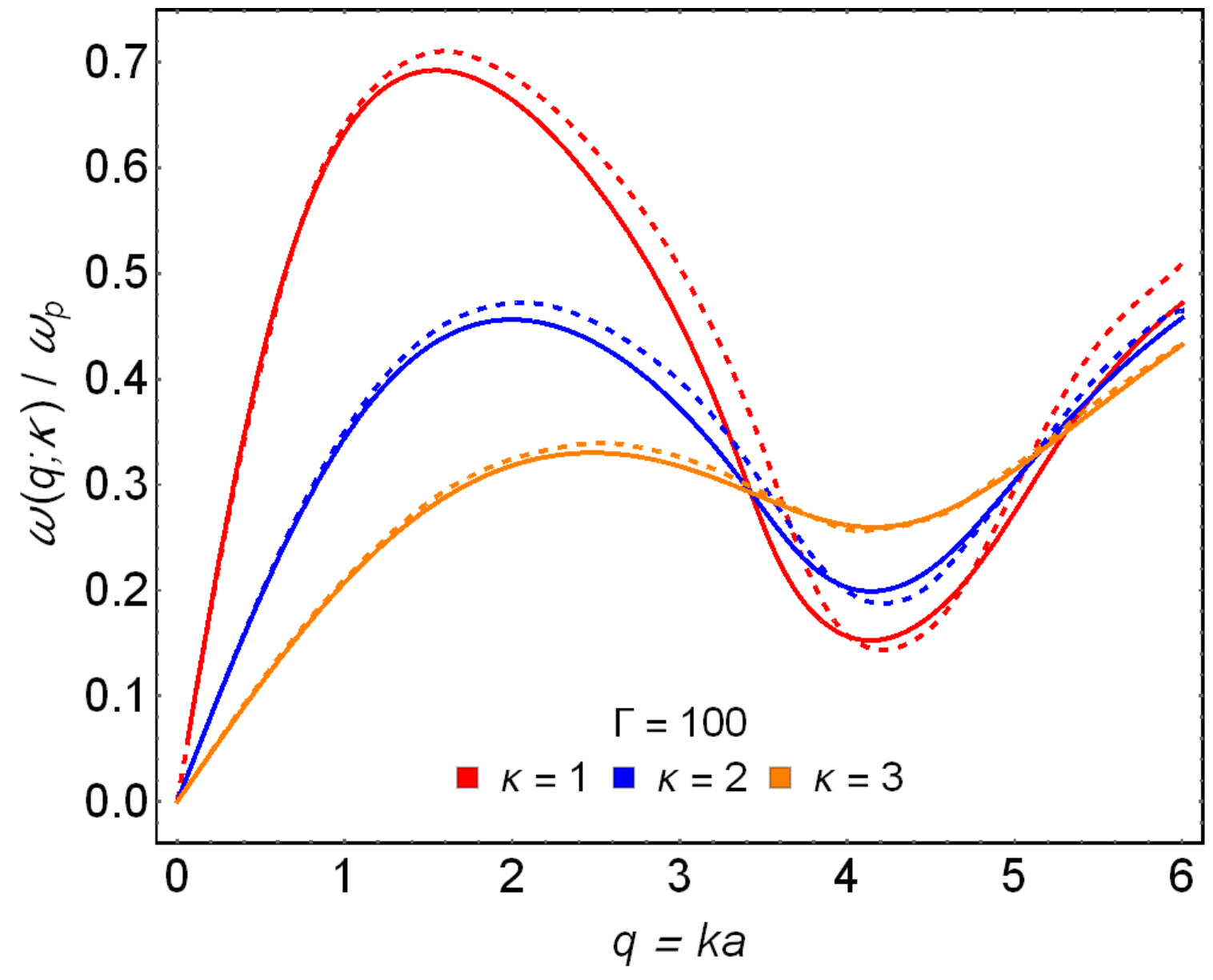

Figure 4.13. Dispersion relation for the YOCP mode. Solid lines represent the exact solution of (4.9), dashed lines represent the exact solution of (4.9), but with moments, calculated from MD DSFs of [32]. 


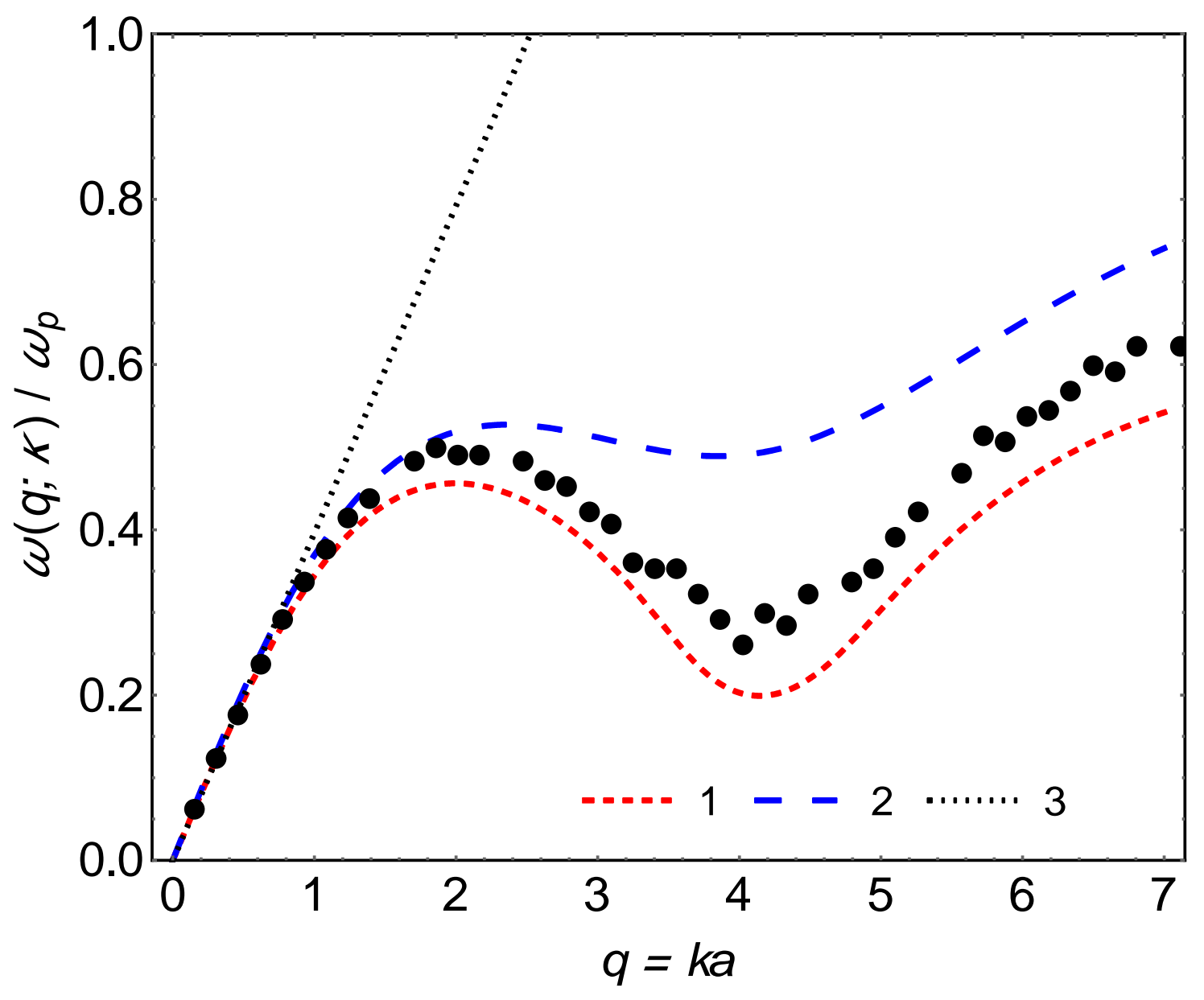

Figure 4.14. Dispersion relation for the YOCP quasiacoustic mode, compared to MD data [32] (dots) at $\Gamma=100$ and $\kappa=2$.

1 stands for the exact solution of (4.9); 2 represents $\omega_{2}(q ; \kappa) ; 3$ is the acoustic part of the mode, $\omega=c_{s} q$, where $c_{s}$ is the sound speed at these conditions. 


\subsection{Dynamic local field corrections.}

We have seen that the NPF plays a significant if not crucial role in the present approach. Generally speaking, it is a non-phenomenological component of the latter. In OCPs it is, additionally, directly related to the dynamic localfield correction (DLFC).

On the other hand, by the definition of the DLFC $G(k, \omega)$,

$$
\varepsilon(k, \omega)=1+\frac{\phi(k) \Pi_{0}(k, \omega)}{1-\phi(k) \Pi_{0}(k, \omega) G(k, \omega)},
$$

where $\Pi_{0}(k, \omega)$ is the RPA polarization operator. Then [34],

$$
G(k, \omega)=1+\frac{1}{\phi \Pi_{0}(k, \omega)}+\frac{\omega^{2}}{\omega_{p}^{2}}-\frac{\omega \omega_{2}^{2}(k)+\omega_{1}^{2}(k) Q_{2}(k, \omega)}{\omega_{p}^{2}\left(\omega+Q_{2}(k, \omega)\right)}
$$

so that any model of the NPF implies a model for the one-component plasma DLFC, and vice versa. Particularly, we have:

$$
G_{0}(k, \omega)=1+\frac{1}{\phi \Pi_{0}(k, \omega)}+\frac{\omega^{2}}{\omega_{p}^{2}}-\frac{\omega^{2} \omega_{2}^{2}+h_{0}^{2} \omega_{1}^{2}}{\omega_{p}^{2}\left(\omega^{2}+h_{0}^{2}\right)}+i \omega h_{0} \frac{\omega_{2}^{2}-\omega_{1}^{2}}{\omega_{p}^{2}\left(\omega^{2}+h_{0}^{2}\right)} .
$$

The following relation between the static LFC and the zero-frequency moment of the loss function follows:

$$
G(k, 0)=1+\frac{1}{\phi \Pi_{0}(k, 0)}-\frac{\omega_{1}^{2}(k)}{\omega_{p}^{2}},
$$

which implies that the static LFC is real and is equivalent to the frequency $\omega_{1}^{2}(k)$ only. Notice also that the DLFC is a response function: it is analytic in the upper half-plane of frequency where $\operatorname{Im} G(k, \omega) \geq 0$. 
Last three expressions are statistics-free, but in a classical system

$$
\phi \Pi_{0}(k, \omega)=\frac{3 \Gamma}{q^{2}}\left[1+\frac{\omega}{k v_{T}} Z\left(\frac{\omega}{k v_{T}}\right)\right]
$$

with $v_{T}=\sqrt{2 / \beta m}$ being the thermal velocity of the particles. So, as it is well known [35], $q=k a$ :

$$
G_{\text {classical }}(q, 0)=1+\frac{q^{2}}{3 \Gamma}\left(1-\frac{1}{S(q)}\right) .
$$

The above Ansatz leads to a reasonable agreement between the DLFC $G_{0}(k, \omega)$ from (4.14) and the simulation data of [33], see Figs. 4.15-4.22. The dots there represent the data of [33] As far as we know, no agreement has been achieved with these data before.

The quantity directly computed in MD simulations of [33] was the intermediate scattering function, $F(k ; t)$, which is the DSF frequency Fourier transform. The $F(k ; t)$ data permitted the authors of [33] to calculate both the DSF and the DLFC, and it was pointed out in [33] that the DLFC was more difficult to compute from the $F(k ; t)$ MD data, than the DSF. The difficulty of determination of the $F(k ; t)$ long-time asymptotic form is reflected in the accuracy of the results of [33] near the zero frequency. Three starting positions are employed to analyze here exactly the DLFC low-frequency behavior: (i) Both the NPF and the DLFC are analytic and holomorphic functions of the complex frequency $z=\omega+$ i $\delta$ in the half-plane $\delta>0$. We assume that they admit Maclaurin expansions at $\omega=0$; (ii) Due to the RieszHerglotz formula for the (Nevanlinna) response functions, the correct zero- 
frequency value of the NPF is purely imaginary, say, ih, with $\mathrm{h}>0$; (iii) The systems studied in [33] are classical, hence

$$
\phi \Pi_{0}(q, \omega \rightarrow 0) \approx \frac{3 \Gamma}{q^{2}}+3 i \sqrt{\pi} \frac{\Gamma}{q^{3}} \frac{a \omega}{v_{T}}+O\left(\omega^{2}\right) .
$$

Thus, we can write

$$
G(q, \omega \rightarrow 0) \approx G(q, 0)-i \omega\left(\frac{\omega_{1}^{2}-\omega_{2}^{2}}{\omega_{p}^{2} h}+\frac{\sqrt{\pi}}{3} \frac{a q}{\Gamma v_{T}}\right)+O\left(\omega^{2}\right),
$$

or, in the another form,

$$
G(q, \omega \rightarrow 0) \approx G(q, 0)-i \omega p(q)+O\left(\omega^{2}\right) .
$$

Then, after some simple calculations, we obtain for the NPF the following limitting form:

$$
R_{2}(q, \omega \rightarrow 0) \approx i \frac{\omega_{2}^{2}(q)-\omega_{1}^{2}(q)}{\omega_{p}^{2}\left(\frac{\sqrt{\pi}}{3} \frac{a q}{\Gamma v_{T}}-p(q)\right)}=i h(q) .
$$

These values are, certainly, different from $h_{0}$. We have processed the graphical material of [33] with respect to the DLFCs and have found that this value of $h$ is quite close to $h_{0}$, and gives satisfactory results for the DSF, see Fig. 4.23. Notice that the expressions for the DSF are depending on $h$ and on $h_{0}$ separately. 


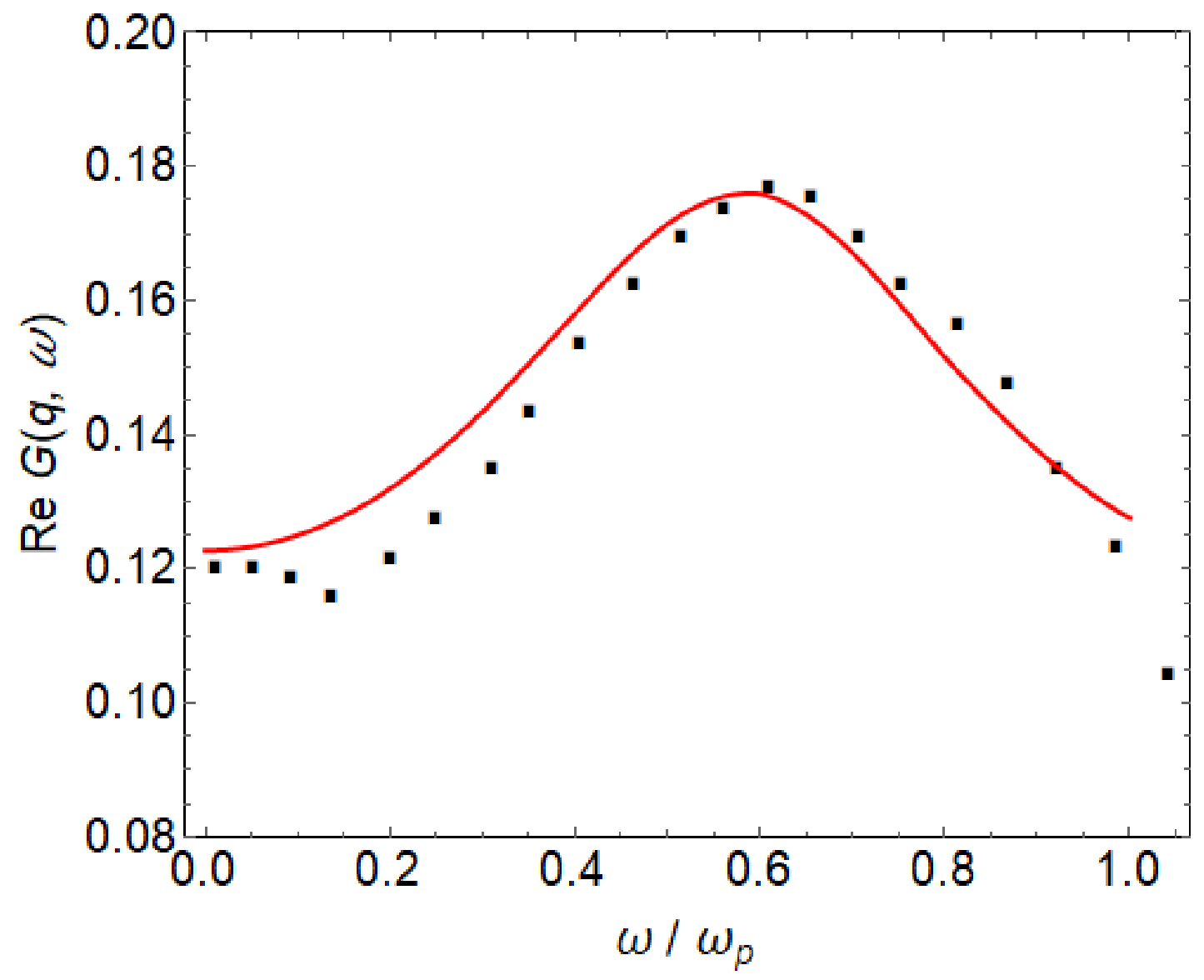

Figure 4.15. Real part of the expression (4.14) for the dynamic local field correction (solid line), compared to the MD results [33] (squares),

$$
\text { at } \mathrm{q}=1.02 \text { and } \Gamma=10 \text {. }
$$




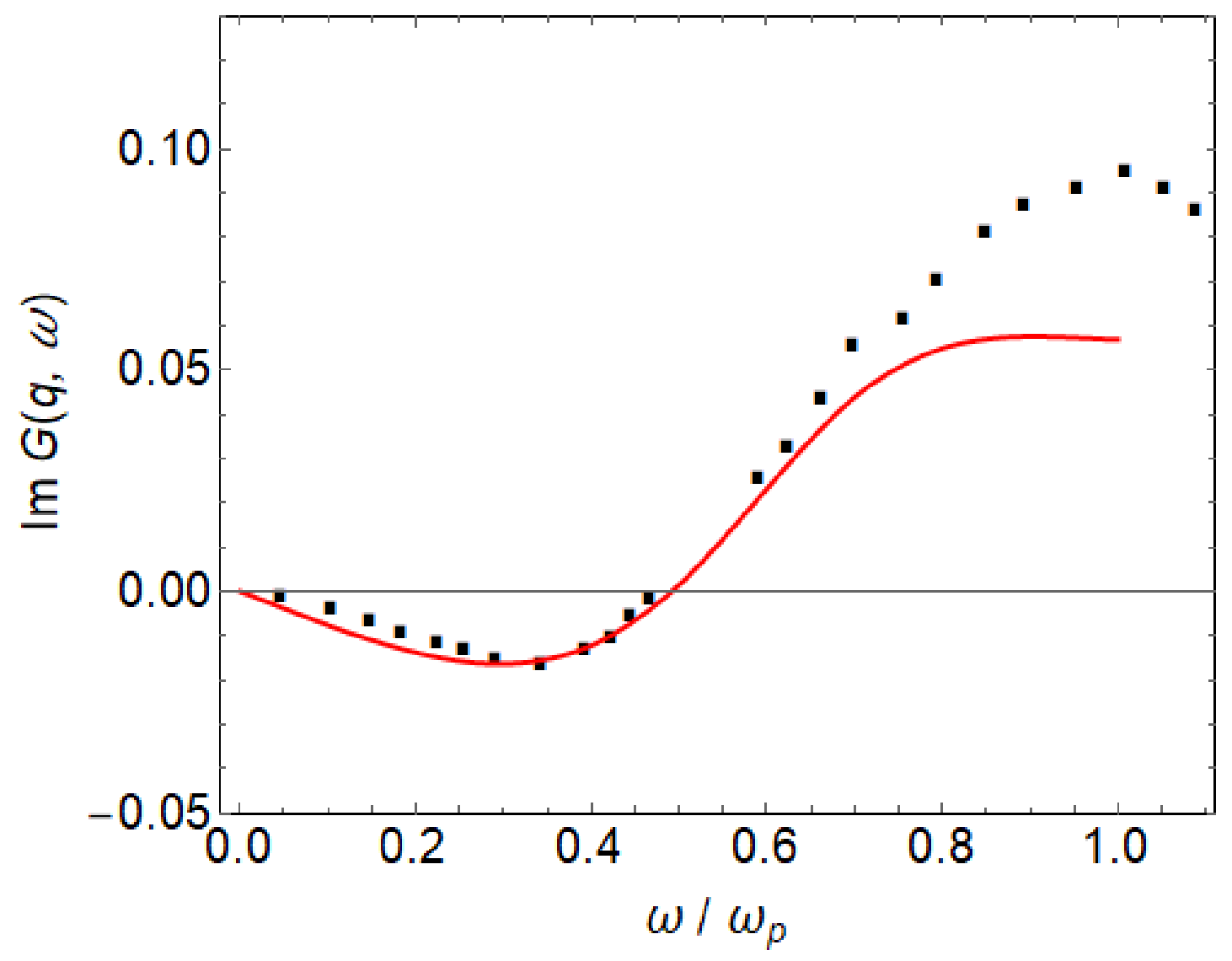

Figure 4.16. Imaginary part of the expression (4.14) for the dynamic local field correction (solid line), compared to the MD results [33] (squares), at $\mathrm{q}=1.02$ and $\Gamma=10$. 


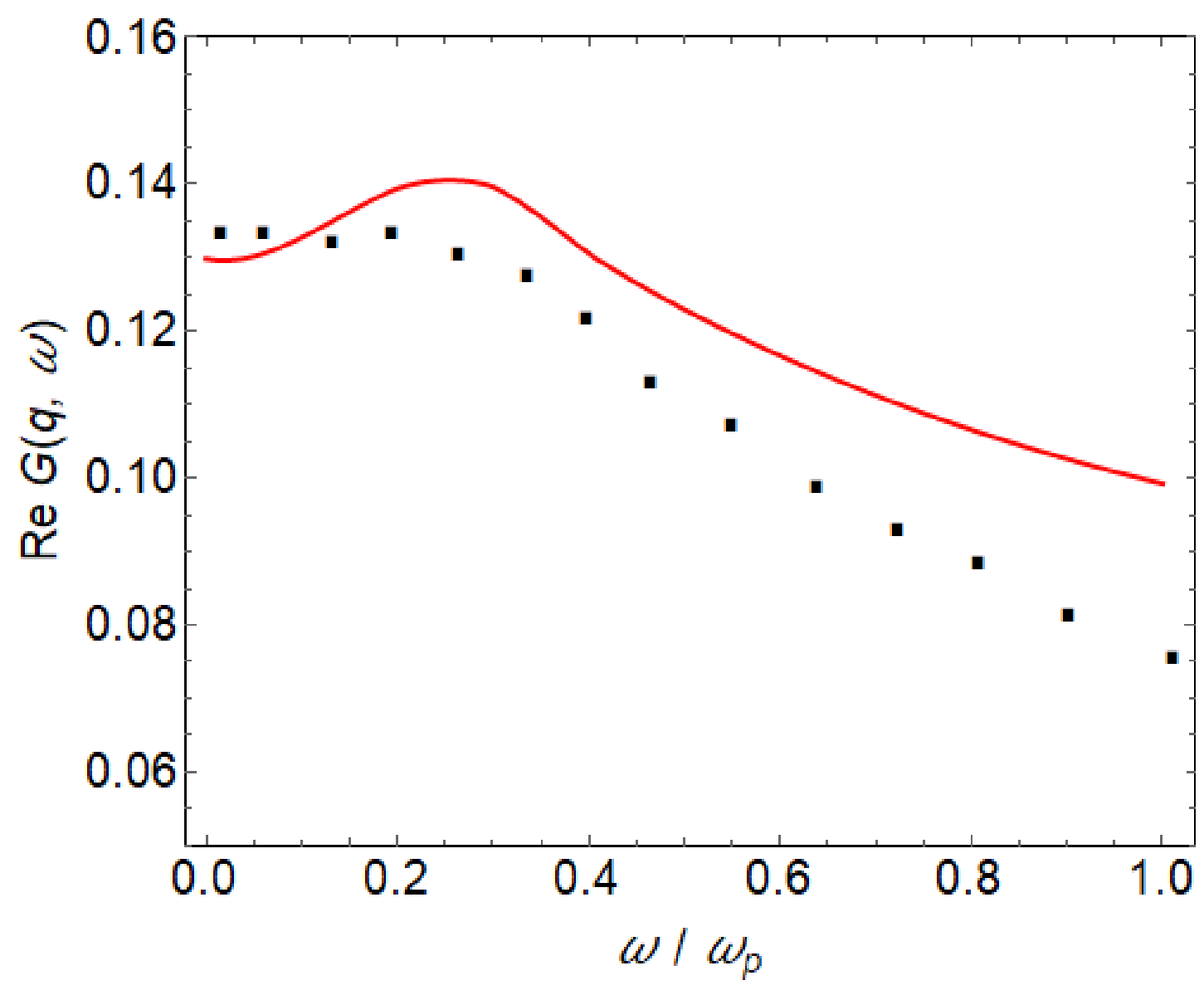

Figure 4.17. Real part of the expression (4.14) for the dynamic local field correction (solid line), compared to the MD results [33] (squares), at $\mathrm{q}=1.02$ and $\Gamma=50$. 


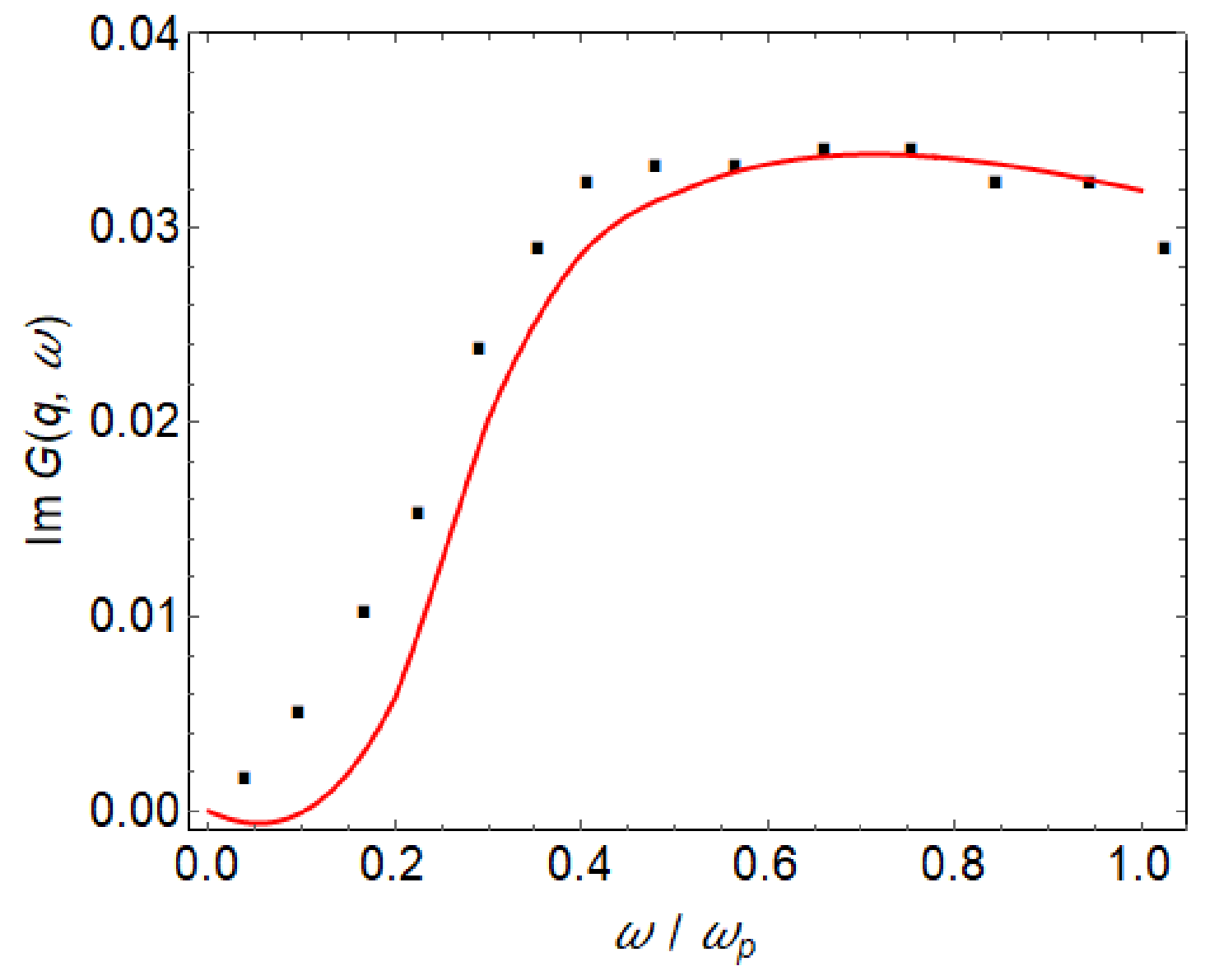

Figure 4.18. Imaginary part of the expression (4.14) for the dynamic local field correction (solid line), compared to the MD results [33] (squares), at $\mathrm{q}=1.02$ and $\Gamma=50$. 


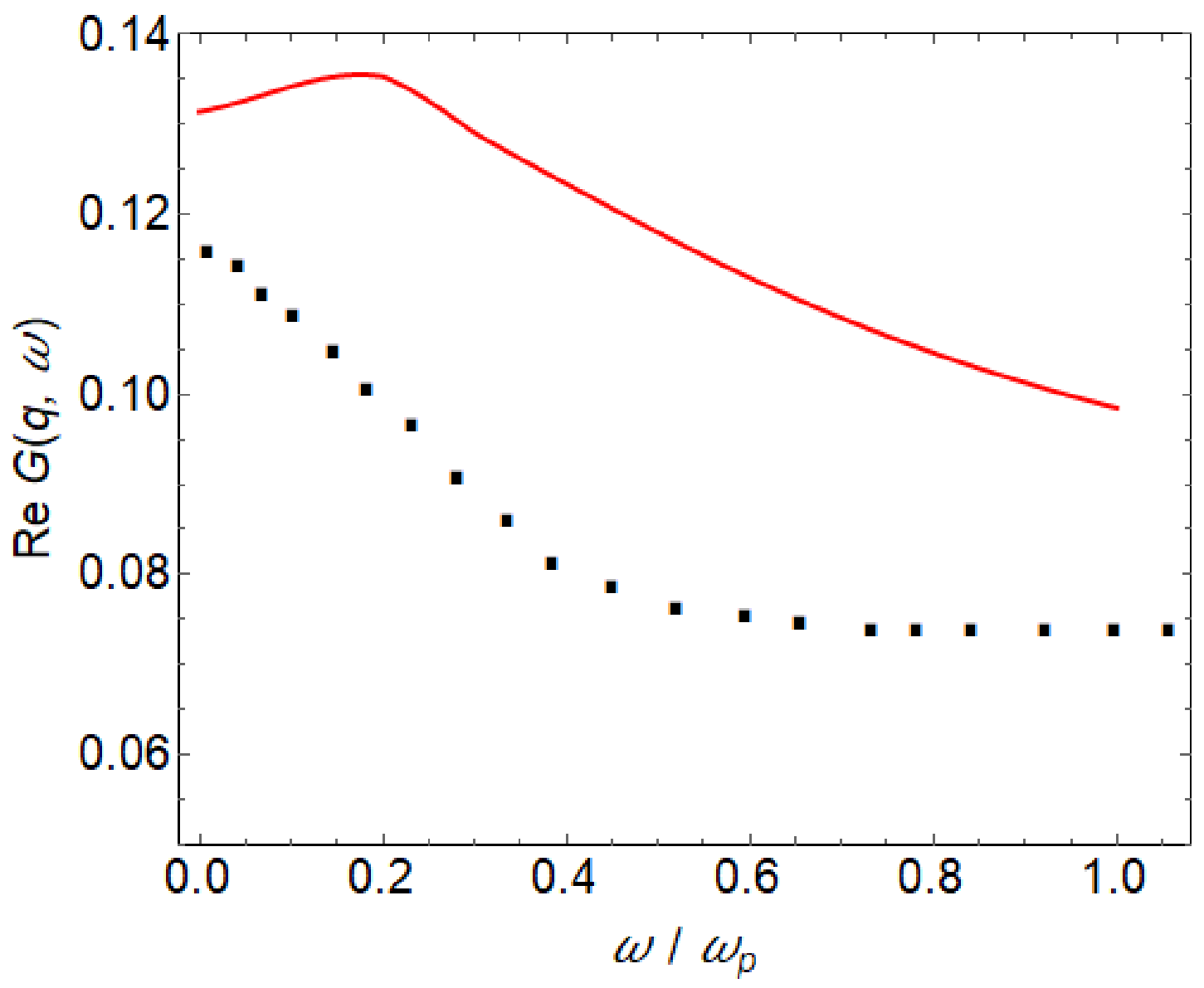

Figure 4.19. Real part of the expression (4.14) for the dynamic local field correction (solid line), compared to the MD results [33] (squares),

$$
\text { at } \mathrm{q}=1.02 \text { and } \Gamma=120 \text {. }
$$






Figure 4.20. Imaginary part of the expression (4.14) for the dynamic local field correction (solid line), compared to the MD results [33] (squares), at $\mathrm{q}=1.02$ and $\Gamma=120$. 


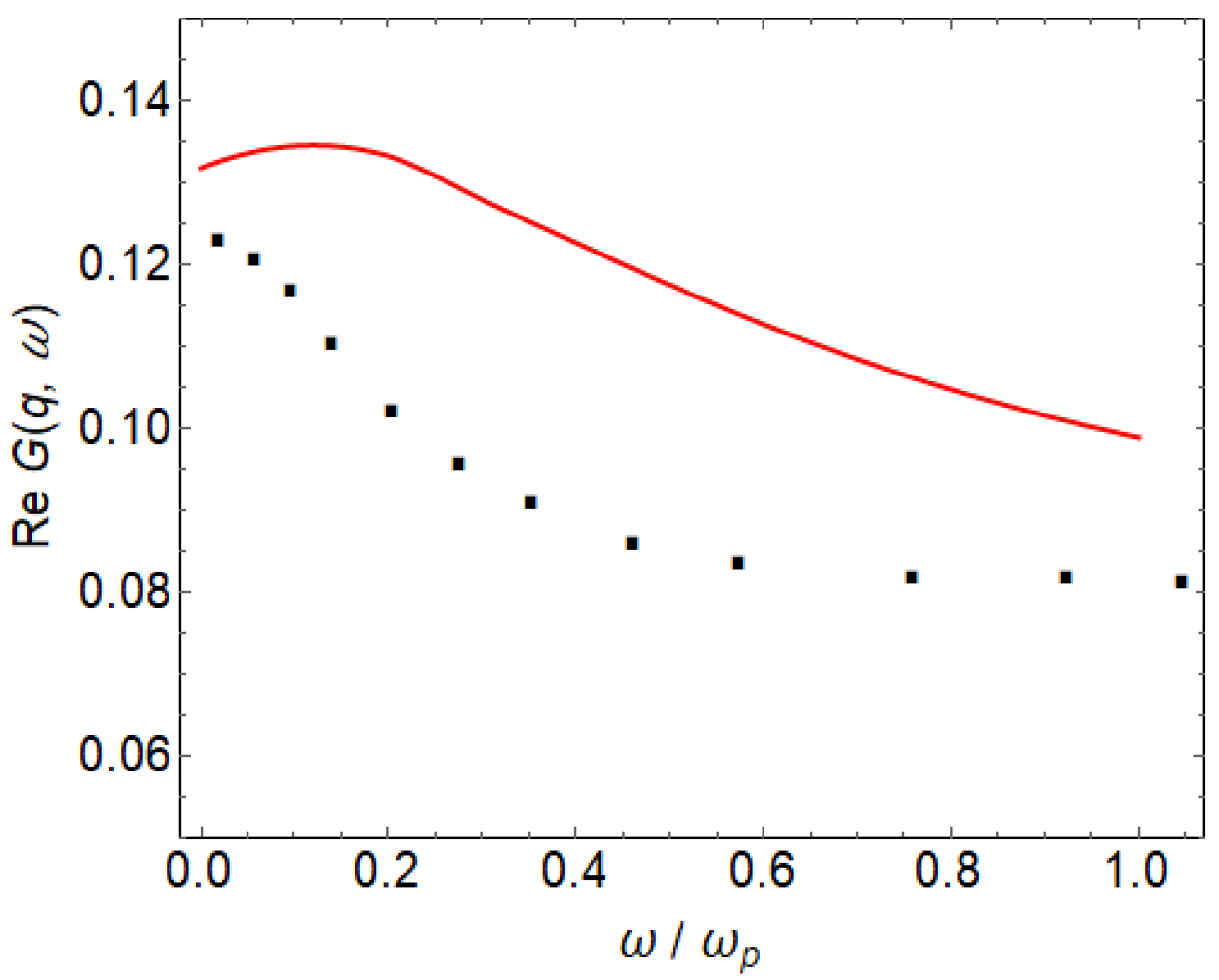

Figure 4.21. Real part of the expression (4.14) for the dynamic local field correction (solid line), compared to the MD results [33] (squares),

$$
\text { at } \mathrm{q}=1.02 \text { and } \Gamma=160 \text {. }
$$




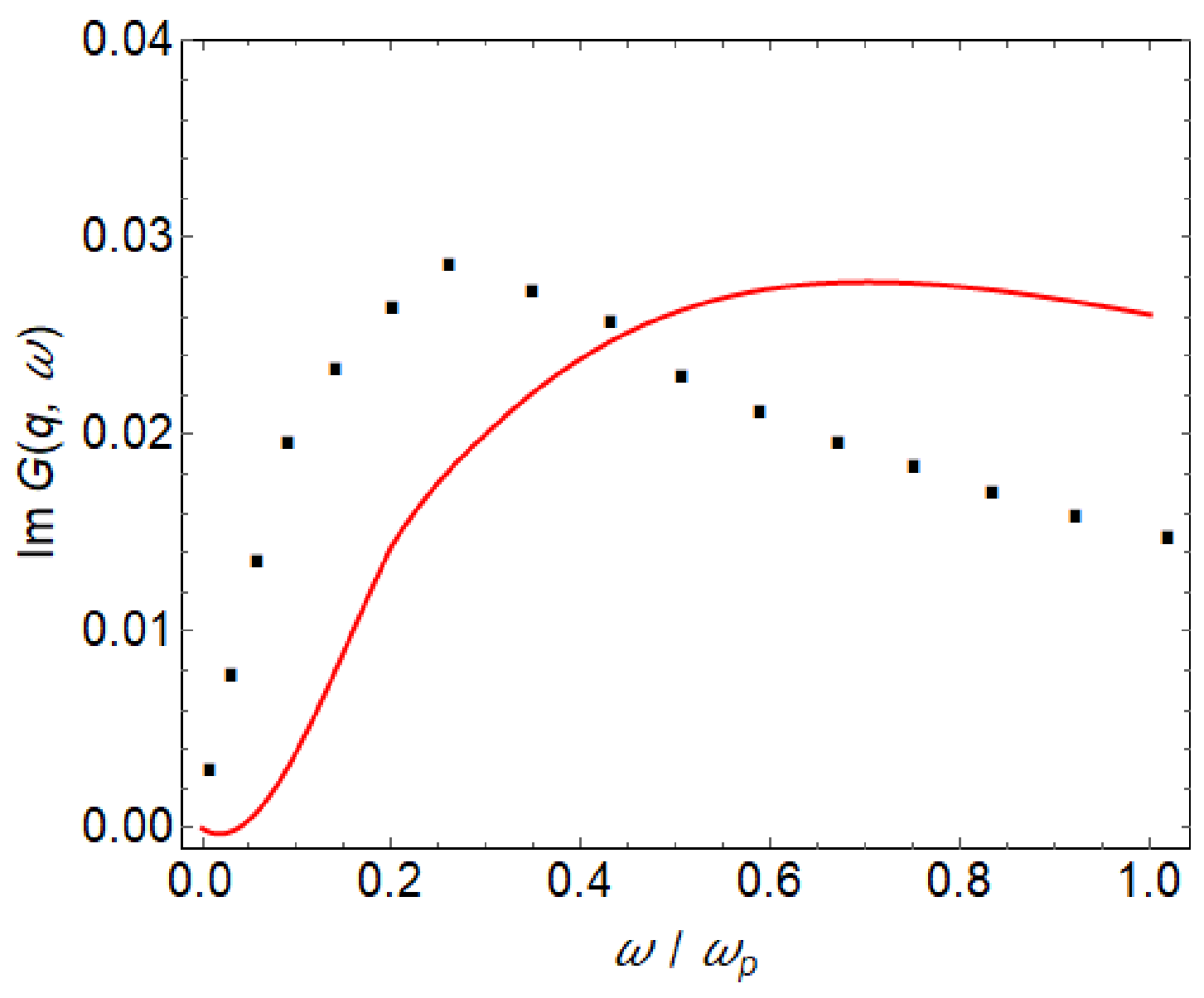

Figure 4.22. Imaginary part of the expression (4.14) for the dynamic local field correction (solid line), compared to the MD results [33] (squares), at $\mathrm{q}=1.02$ and $\Gamma=160$. 

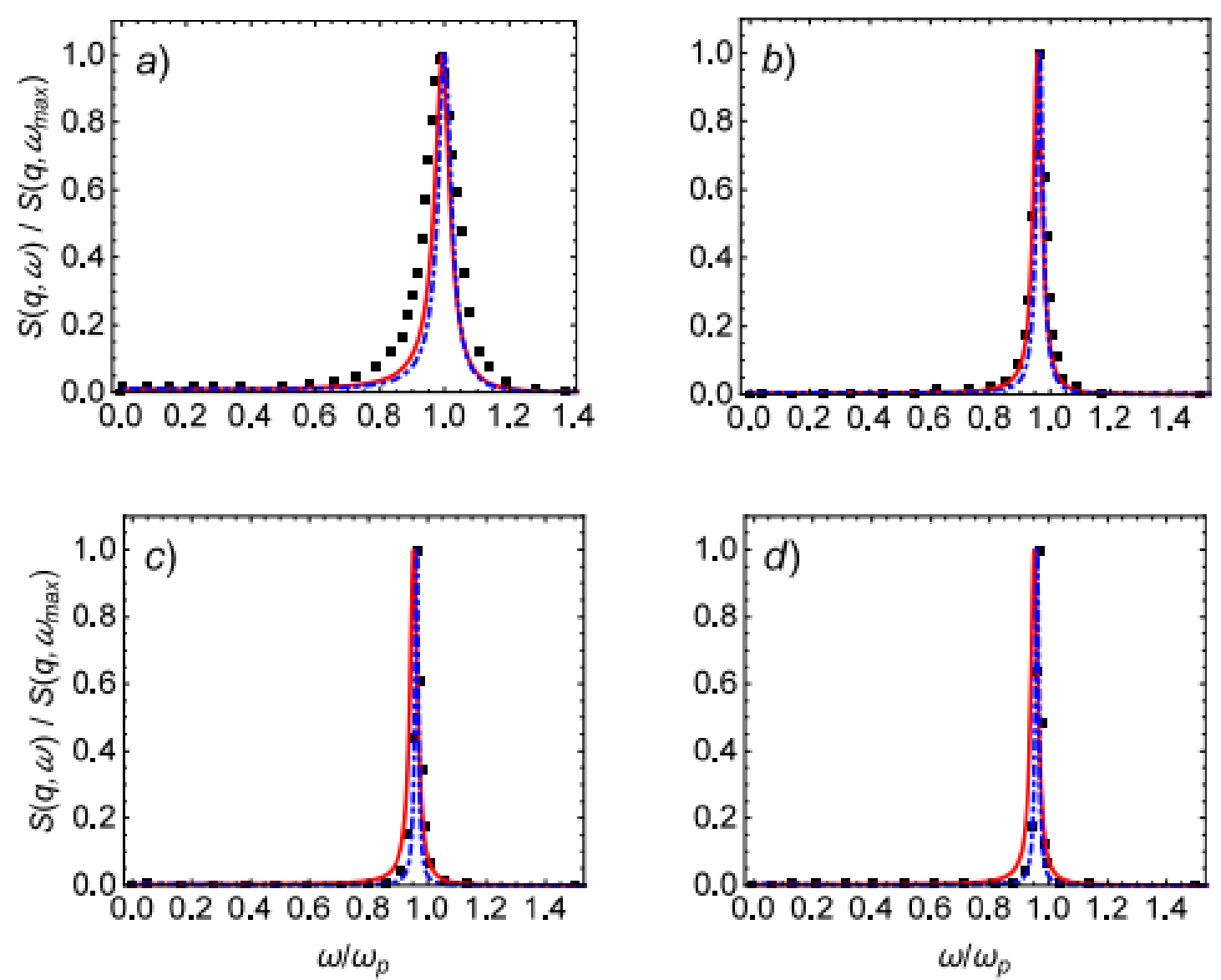

Figure 4.23. Dynamic structure factor (4.7) (lines), normalized to the shifted maxima values in COCPs. Red solid lines - DSFs with $h_{0}(q)$, blue dashed lines - DSFs with $h$ from the procedure (4.21), squares - MD results [33]. All graphs are at $\mathrm{q}=1.02$ and a) $\Gamma=10$; b) $\Gamma=50$; c) $\Gamma=120$; d) $\Gamma=160$. 
We have shown, how the dynamic characteristics of strongly coupled plasmas could be obtained within the non-perturbative model-free moment approach without any data input from simulations so that the inverse dielectric function satisfies the first three nonvanishing sum rules automatically.

The dynamic structure factor and dispersion relation were determined using exclusively the static structure factor calculated from various theoretical approaches.

The expression for the dynamic local field corrections in Coulomb one-component plasmas was obtained in the framework of the algorithm, suggested in [34]. A quantitative agreement is achieved with available simulation data.

In general, the suggested mathematical approach is perfectly applicable in any physical system described by a response function like the inverse dielectric function.

If we employ this approach to study the plasma dielectric function or the dynamic structure factor, the latter are actually the sum rules [35] valid irrespectively of the small-parameter expansions. In this sense, the moment approach is non-perturbative and thus it is auspicious for the determination of dynamic properties of the above crossover systems. Especially, if it is complemented by physically motivated considerations, simplifications or asymptotic considerations.

Though the NPF is not a measurable quantity, it can be determined on the basis of some external, physical arguments or from a NPF corresponding to a broader set of moments. In any case, the quality of such approximations can be finally justified by the quality of the corresponding results compared to the experimental or simulation data. Observe also that the moment approach is equivalent to the continued-fraction method by M.H. Lee and others [36]. 
A new development of the method of moments was suggested recently in [34], (see also the corresponding Supplemental Material [34] (b)), where it was favorably applied to determine various dynamic properties of onecomponent classical strongly coupled Coulomb and Yukawa systems in terms of their static characteristics, without any adjustment to the dynamic simulation data. The validity of the approach was confirmed by a favorable comparison with available simulation data. The robustness of the method was confirmed by applying several schemes of calculation of the plasma static structure factor, which provided results in good agreement with each other, within the precision of the simulations themselves. The main idea of method in [34] was to determine the dynamic characteristics of plasma via its static ones, without any additional parameters.

In next chapter we will analyze several schemes for the calculations of staic characteristics of OCP. 


\section{ANALYSIS OF SCHEMES FOR THE CALCULATION OF PLASMA STATIC CHARACTERISTICS}

As it is laid out in the text, the method of moments complemented by the additional empirical information on the behavior of the plasma dynamic structure factor (DSF), $\mathrm{S}(\mathrm{q}, \omega ; \kappa)$, near the origin $\omega=0$ permitted in both cases we considered, Coulomb and Yukawa classical one-component plasmas, COCPs and YOCPs, respectively, to determine the (static) nonphenomenological (Nevanlinna) parameter function and thus reduce the calculation of the plasma dynamic characteristics to the knowledge of the system static structure factor (SSF).

Certainly, the precision of the SSF data influences the level of quantitative agreement of our results with the DSF simulation data. Here we analyze the influence on our results of seven different approaches to the calculation of plasma SSFs and/or the pair distribution function (PDF), including both theoretical (the hyper-netted chain approximation (HNC) [29,30], the modified HNC (MHNC) [31] and the variational modified HNC (VMHNC) [37-39] and those based on the fitting of precalculated data [31].

In what follows we present graphical material which demonstrates that except for the higher wavenumber values, little discrepancy is observed for the plasma dynamic characteristics calculated with the SSF data obtained within these approaches or using the method of molecular dynamics (MD), see Figs. 5.1-5.4. This stability implies the robustness of the present model. 


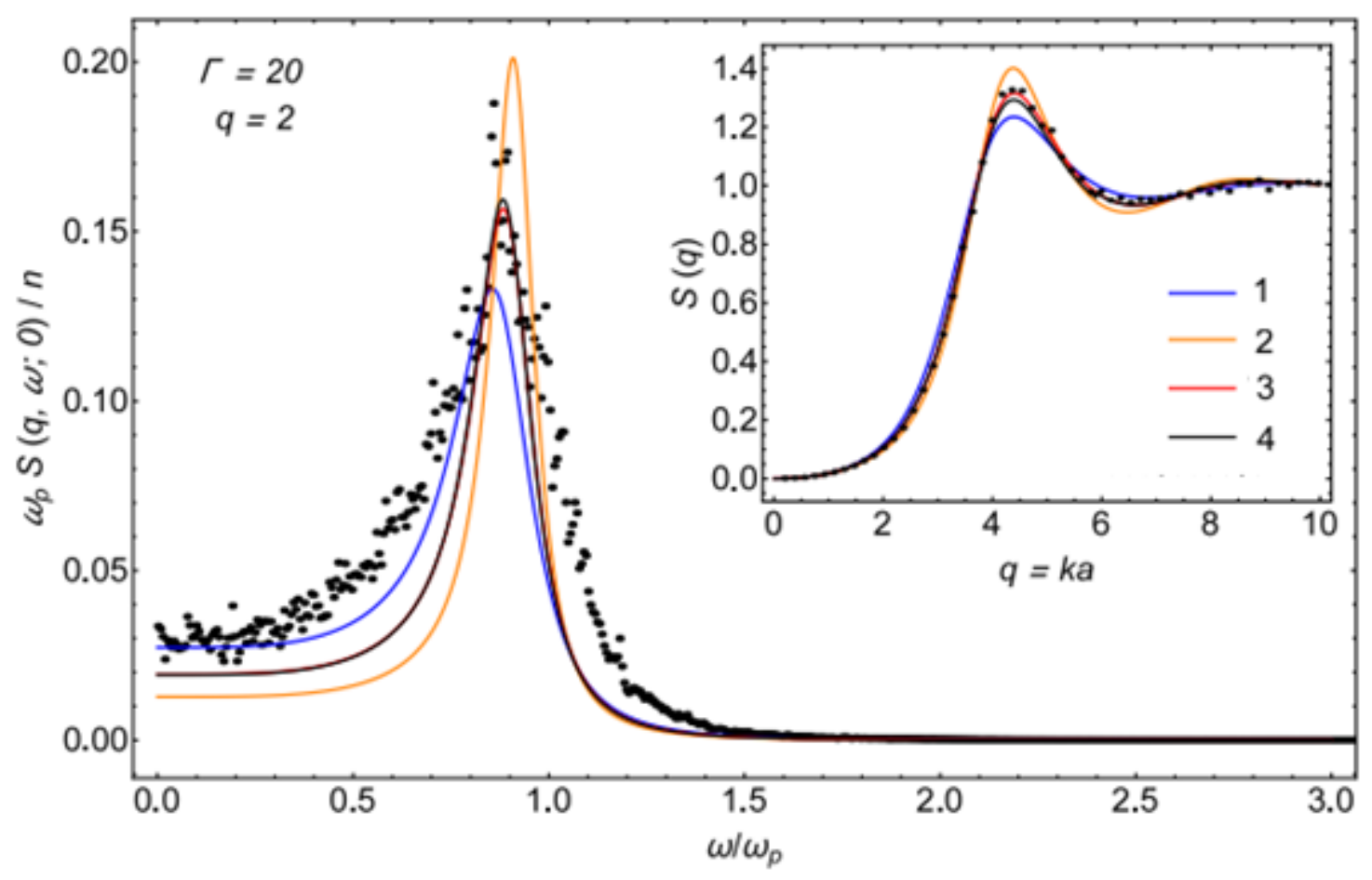

Figure 5.1. Static and dynamic structure factors in COCPs, compared to the MD results (dots) [32]. The static structure factors were calculated using the following schemes: $1-[30], 2-[37], 3-[38,39], 4-[31]$. 


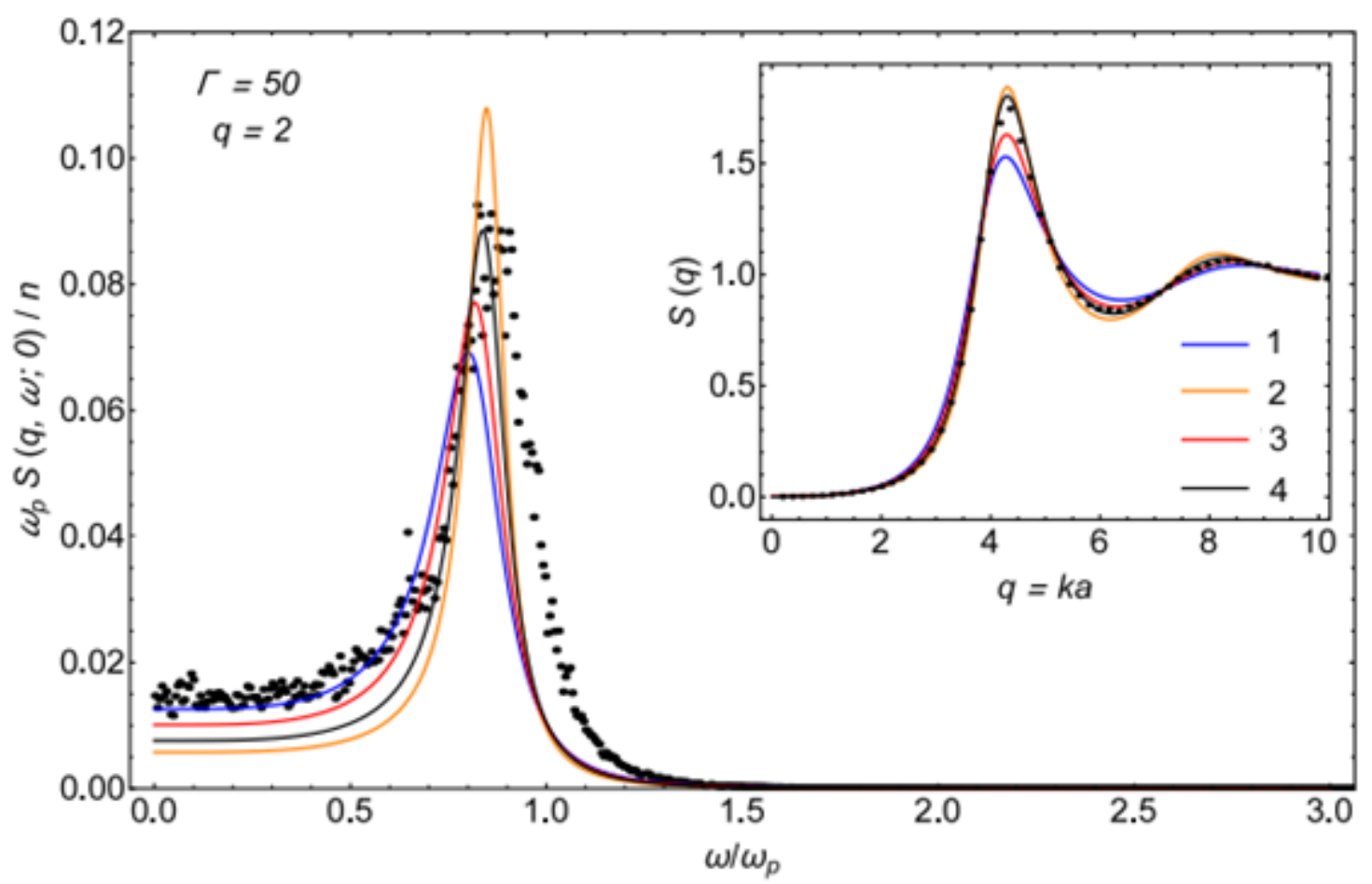

Figure 5.2. Static and dynamic structure factors in COCPs, compared to the MD results (dots) [32]. The static structure factors were calculated using the following schemes: $1-[30], 2-[37], 3-[38,39], 4-[31]$. 


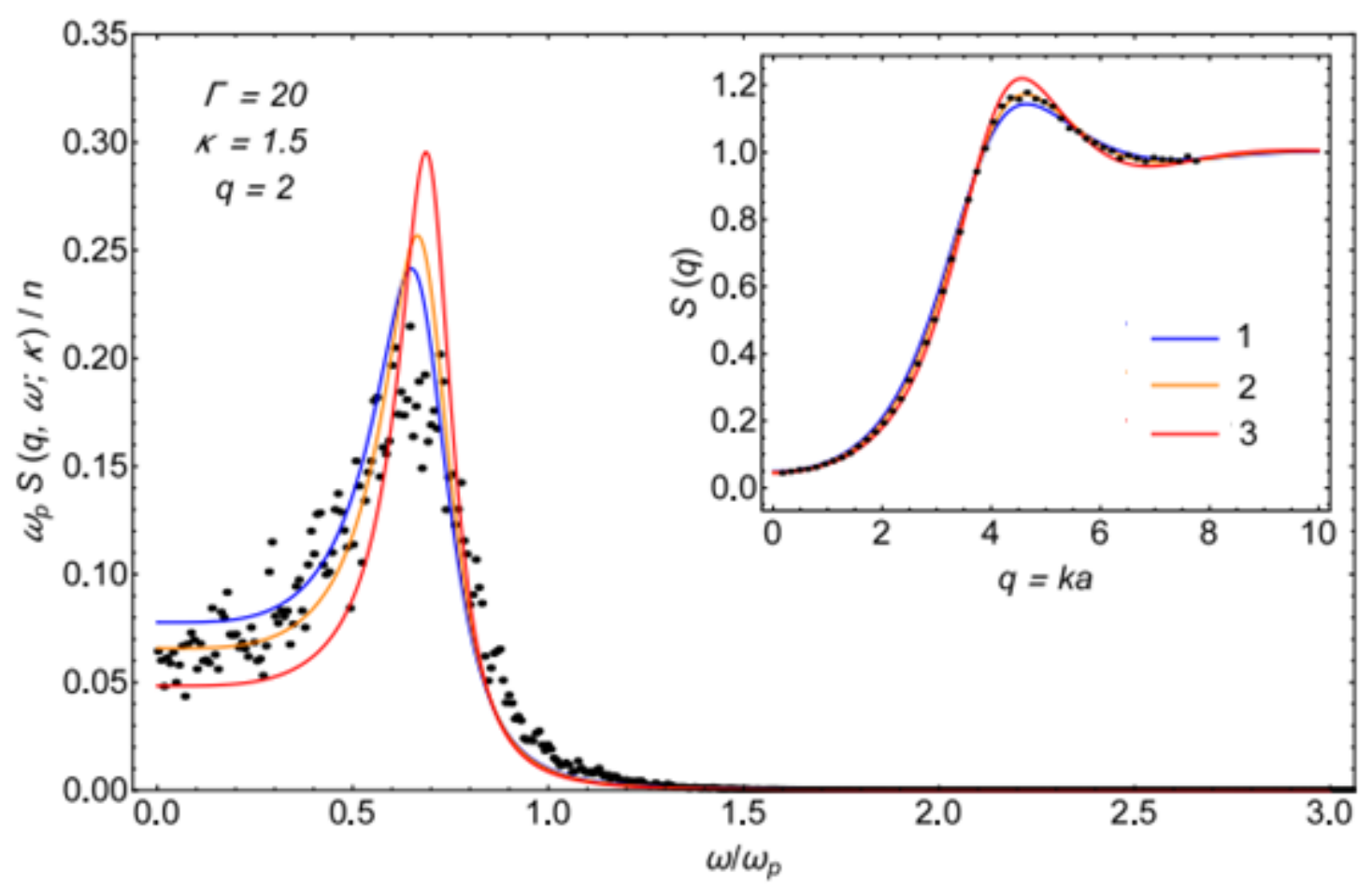

Figure 5.3. Static and dynamic structure factors in YOCP, compared to the MD results (dots) [32]. The static structure factors were calculated using the following schemes: $1-[30], 2-[37], 3-[38,39], 4-[31]$. 


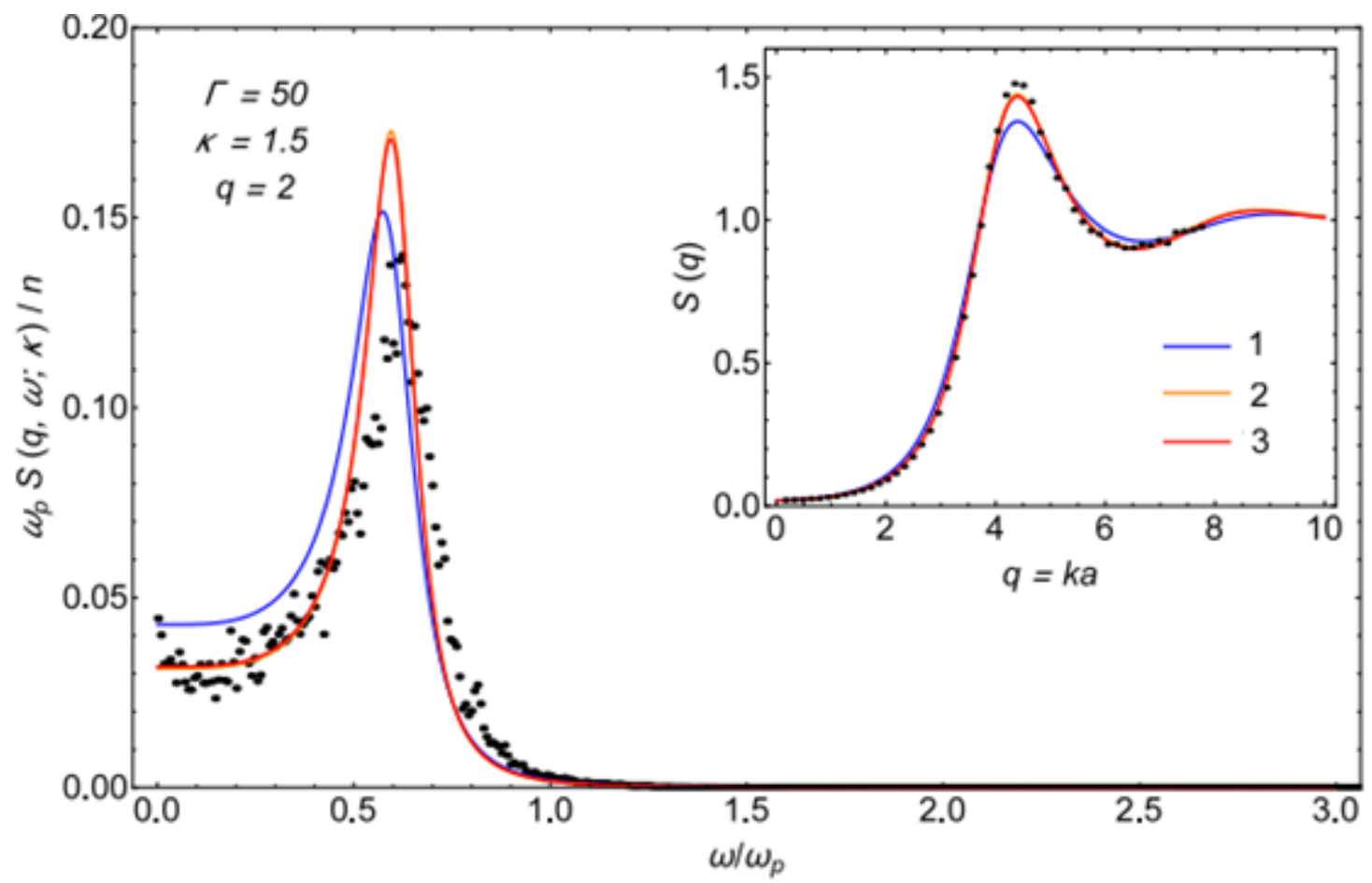

Figure 5.4. Static and dynamic structure factors in YOCP, compared to the MD results (dots) [32]. The static structure factors were calculated using the following schemes: $1-[30], 2-[37], 3-[38,39], 4-[31]$. 
The Fig. 5.5 provides the values of the discrimination parameter $\theta(k)$, which was introduced in the second chapter:

$$
\theta(k)=\frac{2 \omega_{1}(k)-\omega_{2}(k)}{\omega_{p}} \in\left(-\frac{\omega_{2}(k)}{\omega_{p}}, \frac{\omega_{2}(k)}{\omega_{p}}\right) .
$$

We have shown that in case of $\theta(k)>0$ the collective mode propagates in the system, while the dissipation processes quench it when $\theta(k)<0$.

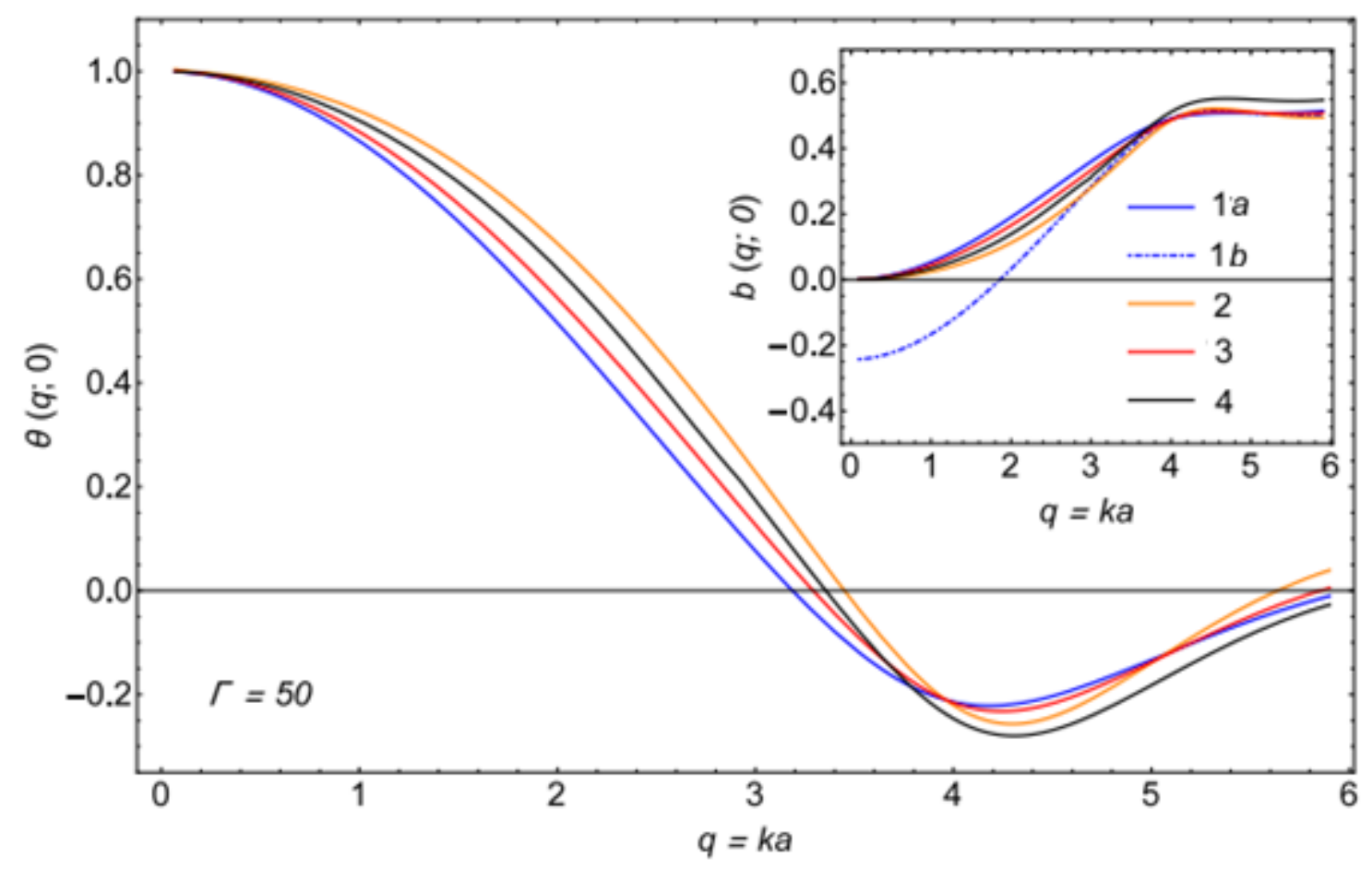

Figure 5.5. The discrimination parameters $\theta(q)$ and $b(q)$ for COCP. The static structure factors were calculated using the following schemes: 1a [29], 1b - [30], 2 - [37], 3 - [38,39], 4 - [31]. 
There is an inserted graph of Cauchy-Schwarz inequality in Fig. 5.5, which we also introduced in the second chapter:

$$
b(k)=\frac{\omega_{2}(k)-\omega_{1}(k)}{\omega_{p}}>0 .
$$

We used this condition for the static schemes analyzing. At higher values of the coupling parameter $\Gamma$ we have probed to use the $\mathrm{HNC}$ method corrected by the bridge function [30]. This was found, however, to lead to the violation of the Cauchy inequality manifested by the relation between the fourth and the lower-order DSF frequency moments. Notice that the DSF is proportional to the parameter $b(k)$, and only in this case, when $b(k)>0$, by virtue of Nevanlinna 's theorem [26], it satisfies the involved sum rules automatically.

We analyzed several well-known schemes for the calculation of SSFs. The overall conclusion is that for our aims the HNC SSFs are quite sufficient, and this is why in the present work we basically employ exactly this classical method. Indeed, relative deviations of the DSF peak position calculated within three different theoretical methods of evaluation of the SSF, from those provided by the dynamic MD calculations. It is observed that the discrepancies between the values of the collective mode frequencies $(\sim 5 \%)$ is comparable to the precision of the dynamic MD data.

Besides, even using sophisticated computers we cannot avoid significant prolongation of calculations within the MHNC and VMHNC schemes, especially, for higher values of $\Gamma$, while the $\mathrm{HNC}$ immediately and consistently leads to quite satisfactory agreement for both systems we deal with here. Nevertheless, further progress in the determination of the SSF and other static characteristics in both systems is needed to determine the realm of applicability of the present model. 


\section{NEVANLINNA PARAMETER FUNCTION INVESTIGATION}

As we have seen, the self-consistent moment approach is quantitatively suitable for the description of dynamic properties of classical OCPs. It is shown in [40] how the method can be successfully extended to the partially or completely degenerate electron gases. Nevertheless, the simplification (33) effectively limits the applicability of the method in the low-coupling regime where the Landau collisionless damping is usually described within the RPA. In other words, we wish to choose a model expression for the NPF capable of incorporating the low- $\Gamma$ RPA-like behavior into the moment scheme.

Here, we consider three different model expressions for the dynamic renormalized NPF $Q_{v}=Q_{2}(\omega ; q)$, both for classical and partially degenerate systems.

We start observing that the loss function effectively depends only on dimensionless variables

$$
x=\frac{\omega^{2}}{\omega_{p}^{2}}, x_{j}=\frac{\omega_{j}^{2}}{\omega_{p}^{2}}, j=1,2 .
$$

I. This implies that the dimensionless NPF can be written as

$$
\frac{Q_{2}(\omega ; q)}{\omega_{p}}=\sqrt{\frac{x}{x_{0}}} X\left(\frac{x}{x_{0}}\right)+i Y\left(\frac{x}{x_{0}}\right)
$$


where $\mathrm{x}_{0}$ is the characteristic value of the new variable equal to $k^{2} v_{t h}^{2}=$ $2 q^{2} / 3 \Gamma$ or $k^{2} v_{F}^{2}=q^{2} v_{F}^{2} / a^{2}$, respectively, in the classical and quantummechanical cases, vth and $\mathrm{vF}$ being, certainly, the thermal and Fermi velocities. Indeed, then the frequency-dependent part of the r.h.s. of (5.2) can be written as a function of $\mathrm{y}=\mathrm{x} / \mathrm{x}_{0}$ and from the condition of the loss function extremum at the point $\mathrm{y}=0$ we obtain:

$$
h=\frac{x_{2}}{\sqrt{x_{1}}} \frac{1}{\sqrt{W_{1}\left(2 W_{1}-W_{2} x_{1}\right)}-W_{0} \sqrt{x_{1}}},
$$

Observe that if $\mathrm{W}_{1}=1, \mathrm{~W}_{0}=0, \mathrm{~W}_{2}=0$, we return to the "static" approximation $h=\frac{x_{2}}{\sqrt{2 x_{1}}}=h_{0}$.

II. Presume first that the NPF is the plasma dispersion function [26]:

$$
\frac{Q_{2}(\omega ; q)}{\omega_{p}}=\sqrt{\frac{x}{x_{0}}} X\left(\frac{x}{x_{0}}\right)+i Y\left(\frac{x}{x_{0}}\right)=\frac{h}{\sqrt{\pi}} Z\left(\frac{\omega+i 0^{+}}{q \sqrt{2 /(3 \Gamma)}}\right),
$$

where $Z\left(\zeta=\xi+i 0^{+}\right)=\mathrm{i} \sqrt{\pi} \exp \left(-\xi^{2}\right)-2 F(\xi)$, with the Dawson integral

$$
F\left(\frac{x}{x_{0}}\right)=\sqrt{\frac{x}{x_{0}}} \exp \left(-\frac{x}{x_{0}}\right) \int_{0}^{1} \exp \left(\frac{x s^{2}}{x_{0}}\right) d s
$$


A simple variable substitution leads to the following representation of $h$ :

$$
h=\frac{x_{2} \sqrt{x_{0}}}{\sqrt{x_{1}}} \frac{\pi \sqrt{x_{1}+2 x_{0}}-2 \sqrt{\pi x_{1} x_{0}}}{2 \pi x_{0}+\pi x_{1}-4 x_{0} x_{1}} .
$$

Alternatively, we might introduce an adjustable parameter and redefine:

$$
\frac{Q_{2}(\omega ; q)}{\omega_{p}}=\sqrt{\frac{x}{x_{0}}} X\left(\frac{x}{x_{0}}\right)+i Y\left(\frac{x}{x_{0}}\right)=\frac{i h}{\alpha+\frac{i(\alpha-1)}{\sqrt{\pi}} Z\left(\frac{\omega+i 0^{+}}{q \sqrt{2 /(3 \Gamma)}}\right)},
$$

Somewhat more cumbersome but straightforward calculations lead in this case to

$$
h=\frac{x_{2}}{\sqrt{x_{1}}} \frac{\sqrt{\pi x_{0}}}{\sqrt{2 \pi x_{0}-(1-\alpha)(\pi+4(1-\alpha)) x_{1}}-2(1-\alpha) \sqrt{x_{0} x_{1}}},
$$

which tends to $\mathrm{h}_{0}$ when $\alpha \rightarrow 1$. Notice that the parameter $\alpha$, generally speaking, can be fixed by the Shannon-entropy maximization procedure [26]. The numerical data with respect to the above frequency-dependent NPFs is presented below, see Figs. $6.1-6.4$. 


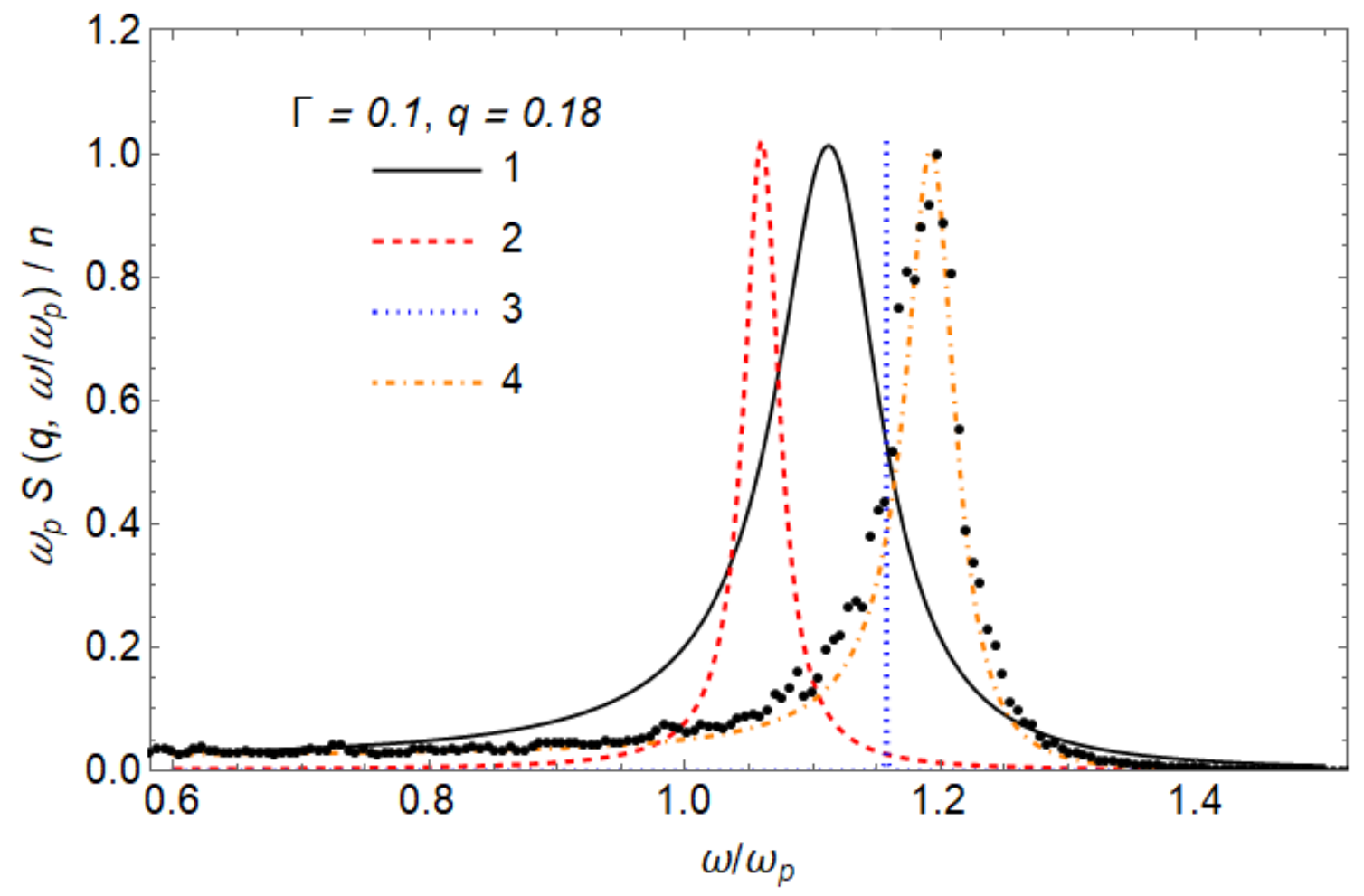

Figure 6.1. Dynamic structure factor fot the COCP presenting the method of moments (MM) data vs. the MD data (dots) [32].

1 - MM with $Q_{2}(q) / \omega_{p}=i h_{0}, 2-\mathrm{MM}$ with $Q_{2}(q) / \omega_{p}$ from (6.4b), with $\alpha=0.5,3-\mathrm{MM}$ with $Q_{2}(q) / \omega_{p}$ from (6.4a), 4 - RPA theory. 


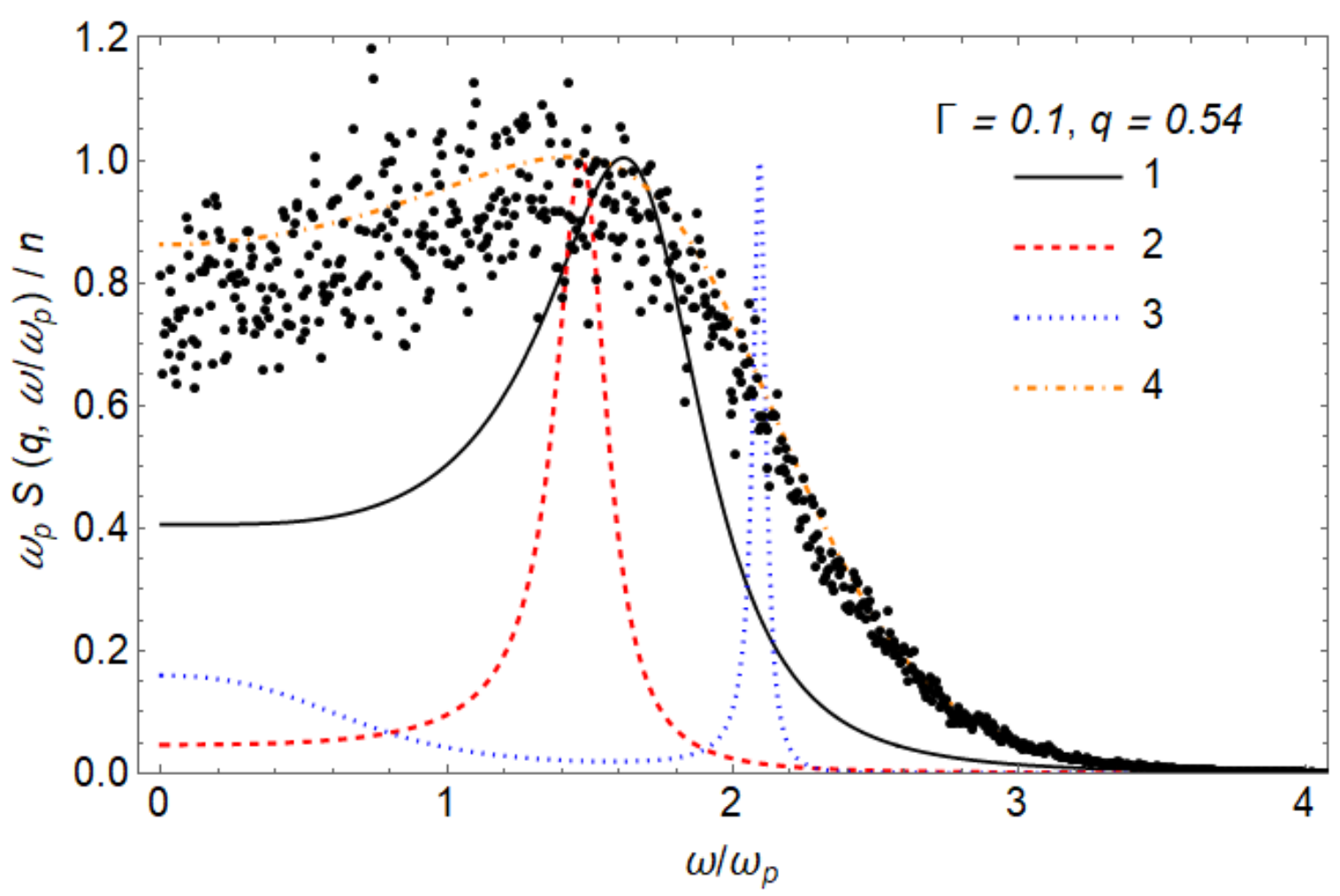

Figure 6.2. Dynamic structure factor fot the COCP presenting the method of moments (MM) data vs. the MD data (dots) [32].

1 - MM with $Q_{2}(q) / \omega_{p}=i h_{0}, 2-\mathrm{MM}$ with $Q_{2}(q) / \omega_{p}$ from (6.4b), with $\alpha=0.5,3-\mathrm{MM}$ with $Q_{2}(q) / \omega_{p}$ from (6.4a), 4 - RPA theory. 


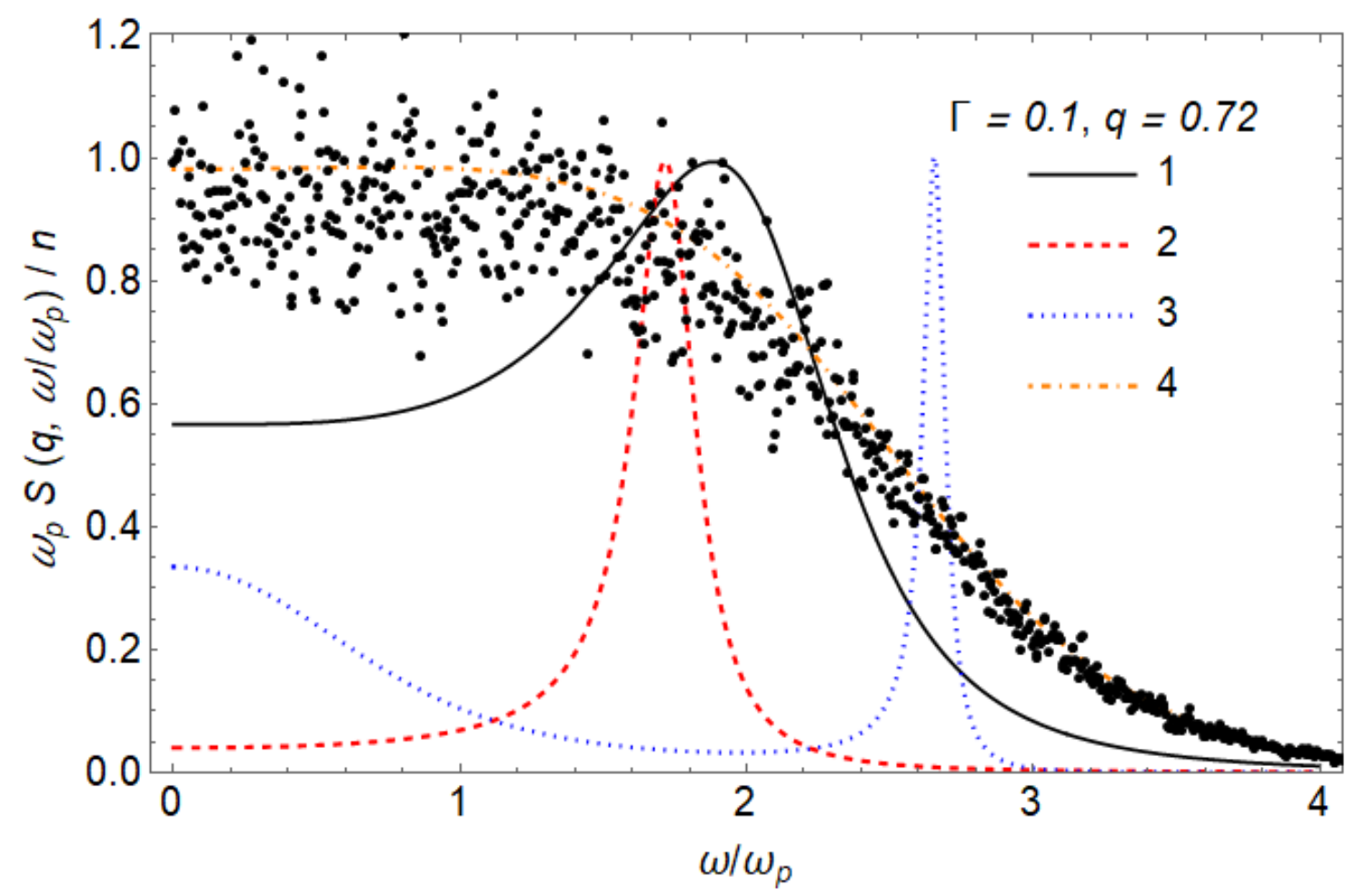

Figure 6.3. Dynamic structure factor fot the COCP presenting the method of moments (MM) data vs. the MD data (dots) [32].

1 - MM with $Q_{2}(q) / \omega_{p}=i h_{0}, 2-\mathrm{MM}$ with $Q_{2}(q) / \omega_{p}$ from (6.4b), with $\alpha=0.5,3-\mathrm{MM}$ with $Q_{2}(q) / \omega_{p}$ from (6.4a), 4 - RPA theory. 


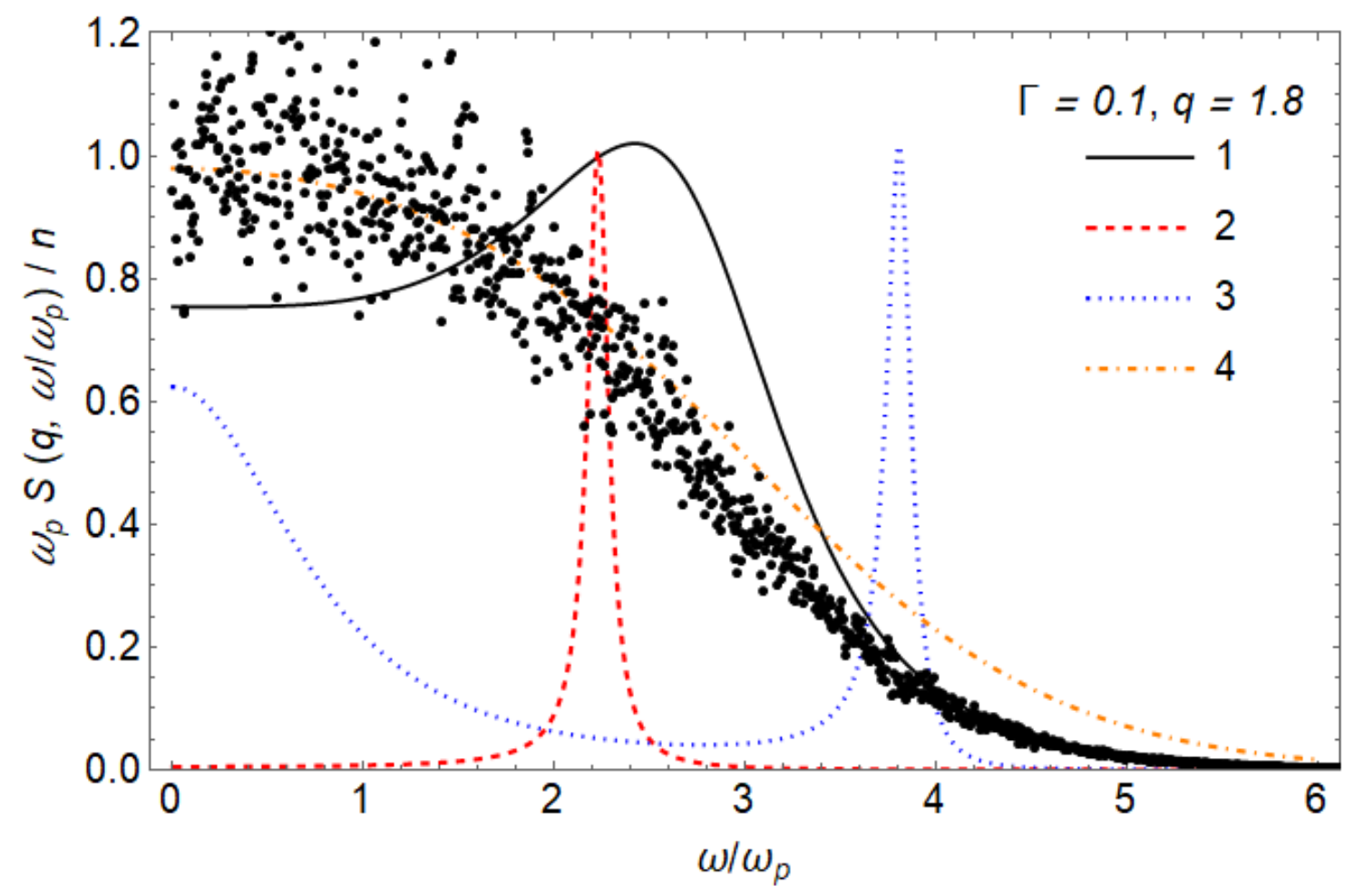

Figure 6.4. Dynamic structure factor fot the COCP presenting the method of moments (MM) data vs. the MD data (dots) [32].

1 - MM with $Q_{2}(q) / \omega_{p}=i h_{0}, 2-\mathrm{MM}$ with $Q_{2}(q) / \omega_{p}$ from $(6.4 \mathrm{~b})$, with $\alpha=0.5,3-\mathrm{MM}$ with $Q_{2}(q) / \omega_{p}$ from (6.4a), 4 - RPA theory. 


\section{CONCLUSIONS}

Dynamic characteristics of dense plasmas are obtained within the nonperturbative model-free moment approach without any data input from simulations so that the inverse dielectric function satisfies the first three nonvanishing sum rules automatically.

The dynamic structure factor, the collective mode dispersion and decay, the sound speed and even the dynamic local-field correction are determined using exclusively the static structure factor calculated from various theoretical approaches or the molecular dynamics. A quantitative agreement is achieved with available simulation data. In general, the suggested mathematical approach is perfectly applicable in any physical system described by a response function like the inverse dielectric function.

It was observed, that the Cauchy-Schwarz inequality yields a simple criterion for validity of the static characteristics. Several different schemes for evaluating the static structure factor of strongly coupled one-component classical plasmas have been tested using the mentioned criterion and some of them have failed to pass.

Several extensions for the NPF representations were investigated. Though the self-consistent method of moments with a static NPF has proven to work very well in warm dense matter, our preliminary results demonstrate that the suggested model NPFs provide a satisfactory agreement with the simulation data in low-density Coulomb plasmas, when the Landau decay plays a significant role. 


\section{A.1. List of publications}

1. Yu. V. Arkhipov, A. B. Ashikbayeva, A. Askaruly, D. Dubovtsev, I. M. Tkachenko, The Plasma Stopping Power Velocity Moment Diagnostics, Contributions to Plasma Physics, v. 54, p. 308-313, 2014. Impact factor: 0.838 .

2. Yu.V. Arkhipov, A. Askaruly, D. Dubovtsev, L.T. Erimbetova, I.M. Tkachenko, Dynamic Collision Frequency in Kelbg-PseudopotentialModelled Plasmas and the Method of Moments with Local Constraints, Contributions to Plasma Physics, v. 55, p. 373-380, 2015. Impact factor: 1.255 .

3. Yu.V. Arkhipov, A.B. Ashikbayeva, A. Askaruly, A.E. Davletov, D. Dubovtsev, I. M. Tkachenko, Screened Effective Interaction Potential for Two-Component Plasmas, Contributions to Plasma Physics, v. 56, p. 403410, 2016. Factor de impacto: 1.44.

4. Yu. V. Arkhipov, A. Askaruly, A. E. Davletov, D. Dubovtsev, Z. Donkó, P. Hartmann, I. Korolov, L. Conde, I. M. Tkachenko, Direct Determination of Dynamic Properties of Coulomb and Yukawa Classical One-Component Plasmas, Phys. Rev. Lett. v. 119, 045001, 2017. Factor de impacto: 8.462 .

5. Yu.V. Arkhipov, A.B. Ashikbayeva, A. Askaruly, M. Bonitz, L. Conde, A.E. Davletov, T. Dornheim, D.Yu. Dubovtsev, S. Groth, Kh. Santybayev, S.A. Syzganbayeva, and I.M. Tkachenko, Sum rules and exact 
inequalities for strongly-coupled one-component plasmas, Contributions to Plasma Physics, 2018, 1-9, https://doi.org/10.1002/ctpp.201700136.

6. Yu. V. Arkhipov, A. B. Ashikbayeva, A. Askaruly, A. E. Davletov, D. Dubovtsev, I. M. Tkachenko, The loss function of dense plasmas and sum rules, International journal of Mathematics and Physics, 2014, v. 5, No. 1, p. 53-59.

7. Yu. V. Arkhipov, A. Askaruly, A. E. Davletov, D. Dubovtsev, L. T. Yerimbetova, I. M. Tkachenko, Effective potential and pressure of twocomponent plasma at high temperature, International journal of Mathematics and Physics, 2014, v. 5, No. 2, p. 48-51.

8. Yu.V. Arkhipov, A. Askaruly, A.E. Davletov, D. Dubovtsev, Z. Donkó, P. Hartmann, I. Korolov, I.M. Tkachenko, Dynamic structure factor of non-ideal one-component plasmas, Physical Sciences and Technology, 2015, v. 2, No. 1, p. 51-54. 


\section{A.2. List of International Conferences}

1. D. Dubovtsev, Dynamic structure factor of Yukawa one-component plasmas, International conference PNP-15, Almaty, Kazakhstan, 2015, Book of Abstracts, p. 32; Effective potentials in semi-classical two-component plasmas, Book of Abstracts, p. 54; Correlation functions in dusty plasmas with finite-size grains, Book of Abstracts, p. 102;

2. D. Dubovtsev, Direct determination of dynamic properties of strongly coupled plasmas, International conference SCCS - 17, Kiel, Germany, 2017, Book of Abstracts, p. 81; Two-component plasma stopping power directly from partial static structure factors, Book of Abstracts, p. 97; Calculating structural characteristics of one-component plasmas, Book of Abstracts, p. 143. 


\section{BIBLIOGRAPHY}

[1] V.M. Adamyan and I.M. Tkachenko, Solution of the Truncated Hamburger Moment Problem According to M.G. Krein // Operator Theory: Advances and Applications. - 2000. - Vol. 118. - P. 33.

[2] M.G. Krein and A.A. Nudel'man. The Markov moment problem and extremal problems. - New York: Providence, 1977.

[3] D. Varentsov, I.M. Tkachenko, and D.H.H. Hoffmann. Statistical approach to beam shaping // Phys. Rev. E. - 2005. - Vol. 71. - P. 066501.

[4] V.M. Adamyan and I. M. Tkachenko. Truncated Hamburger moment problems with constraints // North-Holland Mathematical Studies. -2001. - Vol. 189. - P. 321.

[5] Yu.V. Arkhipov et al., Contr. Plasma Phys. 55, 381 (2015).

[6] M. Urrea, I.M. Tkachenko, and P. Fernández de Córdoba // J. Applied Analysis. - 2001. - Vol. 7. - P. 209.

[7] R.E. Curto, L.A. Fialkow. Recursiveness, positivity, and truncated moment problems // Houston J. Math. - 1991. - Vol. 17. - P. 603-635.

[8] T. Carleman. Sur les fonctions quasi-analytiques // Comptes rendus du Ve Congres des Mathematiciens Scandinaves, 1922. 
[9] T. Carleman. Les fonctions quasi analytiques. Paris: Gauthier-Vilars, 1926.

[10] G.H. Hardy // Messenger of Mathematics. - 1917. - Vol. 47. - P. 175.

[11] R. Nevanlinna, Asymptotische Entwicklungen beschränkter Funktionen und das Stieltjessche Momentenproblem, Suomalaisen tiedeakatemian kustantama, Helsinki, 1922, 1-53; J. Shohat, J. Tamarkin, The Problem of Moments, New York, 1943.

[12] I.M. Tkachenko, Phys. Rev. E 57, 3676 (1998).

[13] S. Ichimaru, Rev. Mod. Phys. 65, 255 (1993);

[14] T. C. Killian, T. Pattard, T. Pohl, and J. M. Rost, Phys. Rep. 449, 77 (2007);

[15] S. Alexander, P. M. Chaikin, P. Grant, G. J. Morales, P. Pincus, and D. Hone, J. Chem. Phys. 80, 5776 (1984);

[16] S. L. Gilbert, J. J. Bollinger, and D. J. Wineland, Phys. Rev. Lett. 60, 2022 (1988);

[17] H. Ohta and S. Hamaguchi, Phys. Rev. Lett. 84, 6026 (2000)

[18] M. S. Murillo, Phys. Plasmas 11, 2964 (2004)

[19] J. Daligault, Phys. Rev. Lett, 119, 045002 (2017). 
[20] J. Daligault, S. D. Baalrud, C. E. Starrett, D. Saumon, T. Sjostrom, Phys. Rev. Lett, 116, 075002 (2016).

[21] T. Dornheim, S. Groth, M. Bonitz, Phys. Rep. 744, 1 (2018).

[22] J. P. Mithen, J. Daligault, B. J. B. Crowley, and G. Gregori, Phys. Rev. E 84, 046401 (2011).

[23] J.P. Mithen, J. Daligault, and G. Gregori, Phys. Rev. E 85, 056407 (2012).

[24] K.N. Pathak, P. Vashishta, Phys. Rev. B 7, 3649 (1973);

[25] V.M. Adamyan, T. Meyer, I.M. Tkachenko, Sov. J. Plasma Phys. 11, 481 (1985); T. Meyer, I.M. Tkachenko, Contrib. Plasma Phys. 25, 437 (1985).

[26] I. M. Tkachenko, Y. V. Arkhipov, and A. Askaruly, The Method of Moments and its Applications in Plasma Physics (Lambert, 2012).

[27] Yu.V. Arkhipov, A.B. Ashikbayeva, A. Askaruly, A.E. Davletov, I.M. Tkachenko, Contr. Plasma Phys. 53, 375 (2013).

[28] Yu. V. Arkhipov, A. Askaruly, D. Ballester, A. E. Davletov, I. M. Tkachenko, G. Zwicknagel, Phys. Rev. E 81, 026402 (2010).

[29] a) I.Z. Fisher, Statistical Theory of Liquids (University of Chicago Press, Chicago, 1964); 
[30] K. Ng, J. Chem. Phys. 61, 2680 (1974).

[31] D.A. Young, E.M. Corey, H.E. DeWitt, Phys. Rev. A 44, 6508 (1991).

[32] Z. Donko et al. Private communications.

[33] J.P. Mithen, J. Daligault, and G. Gregori, Phys. Rev. E 85, 056407 (2012).

[34] Yu. V. Arkhipov, A. Askaruly, L. Conde, A. E. Davletov, Z. Donkó, D. Yu. Dubovtsev, P. Hartmann, I. Korolov, and I. M. Tkachenko. Direct Determination of Dynamic Properties of Coulomb and Yukawa Classical One-Component Plasmas // Phys. Rev. Lett. - 2017. - Vol. 119. - P. 045001.

[35] V.M. Adamyan, T. Meyer, I.M. Tkachenko. RF dielectric constant of a collisional plasma // Sov. J. Plasma Phys. - 1985. - Vol. 11. - P. 481.

[36] J. Hong and M.H. Lee, Phys. Rev. Lett. 55, 2375 (1985); J. Hong and C. Kim, Phys. Rev. A. 43, 1965 (1991).

[37] Y. Rosenfeld, N.W. Ashcroft, Phys.Rev. A 20, 1208 (1979).

[38] M.S. Wertheim, Phys. Rev. Lett. 10, 321 (1963).

[39] G. Faussurier, Phys. Rev. E 67, 046404 (2003); G. Faussurier, Phys. Rev. E 69, 066402 (2004).

[40] Yu. V. Arkhipov, A.B. Ashikbayeva, A. Askaruly, et al., Contr. Plasma Phys., 1-9, (2018). 